
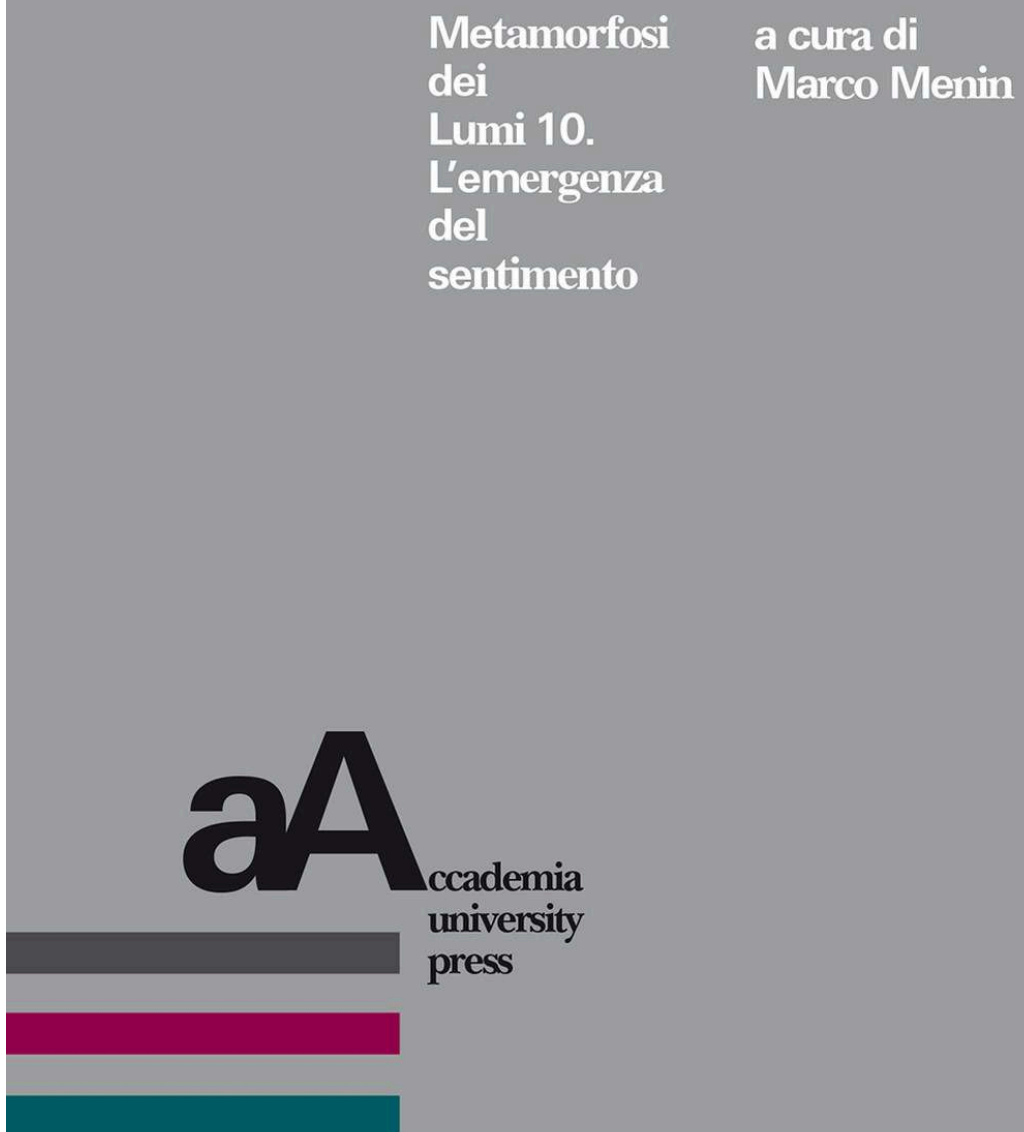


\section{Metamorfosi dei Lumi 10}

L'emergenza del sentimento

\section{Marco Menin (dir.)}

DOI: 10.4000/books.aaccademia.9435

Editore: Accademia University Press

Luogo di pubblicazione: Torino

Anno di pubblicazione: 2020

Data di messa in linea: 21 septembre 2021

Collana: Metamorfosi dei lumi

EAN digitale: 9791280136138

\section{Qboooks}

http://books.openedition.org

\section{Edizione cartacea}

Data di pubblicazione: 1 décembre 2020

EAN (edizione cartacea): 9791280136114

Numero di pagine: 168

\section{Notizia bibliografica digitale}

MENIN, Marco (dir.). Metamorfosi dei Lumi 10: L'emergenza del sentimento. Nouva edizione [online]. Torino: Accademia University Press, 2020 (creato il 23 septembre 2021). Disponibile su Internet: <http://books.openedition.org/aaccademia/9435>. ISBN: 9791280136138. DOI: https://doi.org/ 10.4000/books.aaccademia.9435

(c) Accademia University Press, 2020

Creative Commons - Attribuzione - Non commerciale - Non opere derivate 4.0 Internazionale - CC BYNC-ND 4.0 
METAMORFOSI DEI LUMI

Ricerche interdisciplinari tra Sette e Ottocento

ISSN 2499-8443

collana diretta da

Marco Menin

comitato scientifico

Beatrice Alfonzetti Università "La Sapienza" Roma Michel Delon Université Paris IV-Sorbonne Arnaldo Di Benedetto Università degli Studi di Torino Clara Leri Università degli Studi di Torino Sergio Luzzatto University of Connecticut Enrico Mattioda Università degli Studi di Torino Anne-Marie Mercier-Faivre Université Claude Bernard Lyon 1 Simone Messina Université Lumière Lyon 2 Massimo Mori Università degli Studi di Torino Michael O'Dea Université Lumière Lyon 2 Germana Pareti Università degli Studi di Torino Valeria Ramacciotti Università degli Studi di Torino Chiara Sandrin Università degli Studi di Torino Debora Sicco Università degli Studi di Torino Paola Trivero Università degli Studi di Torino Federico Vercellone Università degli Studi di Torino Klaus Vieweg Friedrich-Schiller-Universität Jena Richard Whatmore University of St Andrews 


\section{CENTRO INTERDISCIPLINARE METAMORFOSI DEI LUMI}

f Metamorfosi-dei-Lumi

1. Esperienze dell'io e creazione letteraria tra Sette e Ottocento a cura di Simone Carpentari Messina

Alessandria, Edizioni dell'Orso, 2000

\section{Tempo, Natura}

a cura di Simone Messina e Valeria Ramacciotti

"Franco Italica», n. 27, 2005, Alessandria, Edizioni dell'Orso

3. Le avventure dell'autorità

a cura di Simone Messina e Valeria Ramacciotti

numero speciale di «Studi Francesi», n. 149, anno L, fasc. II, maggio-agosto 2006

4. L'autorità e le prove della storia

a cura di Simone Messina e Valeria Ramacciotti

Alessandria, Edizioni dell'Orso, 2008

5. II paesaggio

a cura di Simone Messina e Valeria Ramacciotti

Alessandria, Edizioni dell'Orso, 2010

6. Le belle lettere le scienze

a cura di Simone Messina e Paola Trivero

Torino, Accademia University Press, 2012

7. II corpo, la voce, I'eco

a cura di Clara Leri

Torino, Accademia University Press, 2014

8. L'età della storia

a cura di Simone Messina e Valeria Ramacciotti

Torino, Accademia University Press, 2016

9. Segni e metafore dell'Infinito nell'epoca classicistico-romantica

a cura di Chiara Sandrin

Torino, Accademia University Press, 2017

10. L'emergenza del sentimento

a cura di Marco Menin

Torino, Accademia University Press, 2020 
Metamorfosi a cura di

dei

Marco Menin

Lumi 10.

L'emergenza del

sentimento

aA 
Metamorfosi

dei

Lumi 10.

L'emergenza

del

sentimento

Volume realizzato con il contributo

del Dipartimento di Filosofia e Scienze dell'Educazione dell'Università degli Studi di Torino

(C) 2020

Accademia University Press

via Carlo Alberto 55

I-10123 Torino

\section{(c) (i) $(9)$}

prima edizione dicembre 2020

isbn 979-12-80136-11-4

edizioni digitali www.aAccademia.it/lumi10

http://books.openedition.org/aaccademia

book design boffetta.com

Accademia University Press è un marchio registrato di proprietà di LEXIS Compagnia Editoriale in Torino srl 
Parte prima. Sentimento e morale

La riflessività ed oggettività della morale nella ricostruzione sentimentalista di David Hume

Da Jean-Jacques a Julien: Stendhal, Rousseau e la metamorfosi della sensibilità

L'Encyclopédie Méthodique des passions au début du $\mathrm{XIX}^{\mathrm{e}}$ siècle

Parte seconda. Sentimento e retorica

Madame du Deffand: dall'estetica alla retorica dell'emozione

I linguaggi delle emozioni in Inghilterra

tra Sette e Ottocento: arte, poesia e teatro

Musique et émotion au XVIII siècle: quelques regards sur une question sans réponse

Parte terza. Sentimento e politica

Coltivare le emozioni tra solitudine e socialità.

Gli esempi di Shaftesbury e Zimmermann

Strong emotions of terror: emozione e politica in Edmund Burke

Indice dei nomi

Gli autori 

Metamorfosi

Lumi 10.

L'emergenza del

sentimento

\section{Introduzione}

Paola Trivero

«Mes passions m'ont fait vivre, et mes passions m'ont tué»: la confession di Rousseau ben potrebbe inserirsi in un virtuale decalogo dedicato alle peculiarità delle emozioni. Prerogative dell'emozione che sembrerebbero all'apparenza antitetiche all'esaltazione della ragione, notorio e scontato emblema del secolo dei Lumi, mentre sappiamo, anche per il fiorire della più recente esegesi, quanto le emozioni e le manifestazioni del sentimento trovarono un fertile terreno di osservazione nel Settecento, certo sull'onda di Cartesio, Spinoza, Malebranche e Leibniz. Un campo di ricerca che dalla sfera fisiologica passa alla psicologica grazie a un'energia intellettuale volta alla riformulazione di una diversa normativa morale ed estetica, secondo una linea che, se pur innegabilmente debitrice al fondatore della filosofia moderna e a coloro che a lui seguirono, spazia ora oltre i confini della filosofia.

Già sul finire del primo ventennio del secolo, rifacendosi all'assioma ut pictura poësis, Jean Baptiste Du Bos sosteneva, nelle sue Réflexions critiques sur la poésie et la peinture (1719), la basilare autorevolezza del sentimento nelle valutazioni del bello sia esso letterario che artistico. Sottraendo il giudizio estetico alla freddezza logica e implicandovi la sensibilità individuale, Du Bos precisava comunque la misura artificiosa 
Metamorfosi dei Lumi 10. L'emergenza del sentimento

VIII del fattore emotivo dal momento che la riproduzione o poetica o pittorica di un evento coinvolgente provoca sì in chi ne è partecipe un'emozione e una passione tuttavia non vere ma, viceversa, artificiali. Assistendo a uno spettacolo teatrale siamo consapevoli di essere di fronte a una imitazione del reale pur essendone impressionati e, citando il Cid di Corneille, postillava che non ci rechiamo allo spettacolo per vedere veramente Chimène e Rodrigue.

Per tali riflessioni sul teatro, il pensiero va facilmente a quel passo del Paradoxe sur le comédien, là dove Diderot differenziava le lacrime che sgorgano dal cuore (le lacrime dell'«homme sensible») e quelle che scendono dal cervello (le lacrime del «comédien») e poneva la differenza tra l'emozione vera e l'emozione falsa, enunciando il suo 'paradosso' e allontanandosi, pertanto, dalle formulazioni di Du Bos. La bravura, la grandezza di un attore non sta nel sentire delle emozioni reali ma invece nel raffigurarne l'espressione; viceversa l'attore scadente cede all'emozione reale. Dunque: «C'est l'extrême sensibilité qui fait les acteurs médiocres; c'est la sensibilité médiocre qui fait la moltitude des mauvais acteurs; et c'est le manque absolu de la sensibilité qui prépare les acteurs sublimes». Un conto è l'attore nella vita e un altro è l'attore sulla scena e così il philosophe citava la propria reazione la prima volta che vide la celebre attrice Claire Josèphe Hippolyte Léris de La Tude, detta Mlle Clairon, non in scena bensì «chez elle»: "Ah! mademoiselle, je vous croyais de toute la tête plus grande».

Le conclusioni di Du Bos e di Diderot sono sintomatiche delle diverse prospettive con cui gli intellettuali settecenteschi guardarono il manifestarsi delle emozioni secondo, appunto, ottiche e discipline differenti a riprova della poliedricità e delle interazioni intellettuali del secolo.

Precipuamente finalizzato a studiare questo orientamento, il presente volume è suddiviso in tre parti che analizzano il sentimento in rapporto alla morale, alla retorica e alla politica, secondo un percorso che intende testimoniare e convalidare l'indubbia circolazione delle idee a livello europeo: una prerogativa che, da sempre, contraddistingue il Centro Metamorfosi dei Lumi.

Per Sentimento e morale il discorso esamina le sinergie intercorrenti con la filosofia, con la letteratura e con la divulgazione enciclopedica. 
Per la filosofia viene riconsiderata la posizione di David Hume, con una dialettica riflessione sulle argomentazioni portate avanti nel Treatise of Human Nature per ragionare sulle innovative posizioni inerenti la «ricostruzione sentimentalista» del filosofo scozzese per quanto concerne l'etica e approfondirne gli esiti.

Per la letteratura viene valutata l'influenza esercitata da una delle figure più prestigiose dei Lumi, ovvero l'influenza di Jean-Jacques Rousseau sulle generazioni successive soprattutto nell'educazione dei nuovi e giovani scrittori, da Senancour a Hugo, qui su Stendhal, lettore adolescente della Nouvelle Héloïse e dell' Émile. Si tratta di una ascendenza dalla valenza filosofica e singolarmente bifronte: Rousseau è il maestro indiscutibile e, parimenti, è da lui che ci si vuole svincolare. Da una mirata rilettura di Le Rouge et le Noir e da un serrato dialogo con la critica e i dettati rousseauiani, il romanzo si rivela una risposta alla dottrina ed esperienza della sensibilité, una sensibilité declinatasi ormai in sensiblerie e dalla quale Henry Beyle, con la mediazione di Julien Sorel, intende emanciparsi. A corollario resta da aggiungere che Rousseau rimane senza dubbio, pur non essendo oggetto di un'indagine precipua, una sorta di presenza-assenza per tanti della sua e delle successive generazioni.

Sigla la prima parte una ricognizione sulle passioni in base a una capillare catalogazione, e congruente disamina, delle voci presenti negli specifici Dictionnaires della monumentale Encyclopédie Méthodique, quando si registra, nella loro elaborazione, una svolta che può configurarsi come una rottura epistemologica: il discorso da morale, se non moraleggiante o per non dire moralista, si va evolvendo in medico-terapeutico. Si verifica, insomma, un'analisi fenomenologica delle passioni.

Nella seconda sezione, i legami tra Sentimento e retorica riguardano la metamorfosi della comunicazione emozionale; le emozioni intercorrenti tra pittura, poesia e teatro e l'emozione relativa alla musica.

Sullo svelamento degli impulsi emozionali soggettivi e sulla loro trasformazione, il caso di Madame du Deffand risulta sicuramente paradigmatico del tournant des Lumières. Figura chiave di uno dei simboli della fecondità intellettuale del secolo, la salonnière Madame du Deffand passa, nelle proprie lettere, da una posizione decisamente aliena a ogni 
Metamorfosi dei Lumi 10. L'emergenza del sentimento abbandono o cedimento sentimentale a una che rivendica la legittimità di uno stile epistolare incline a esprimere il valore dell'emozione. Un'inversione di tendenza che emerge dal carteggio con il président François Hénault e da quello con Horace Walpole, determinando un rovesciamento dei ruoli: colei che, nello scambio epistolare con il président, ingiungeva il controllo dell'emozione verrà, a sua volta, aspramente sollecitata dall'autore del Castel of Otranto a celare le proprie spinte emotive.

Sempre per Sentimento e retorica, una conferma della intersezione tra le arti è offerta dal contesto inglese quando viene attribuita al teatro la prerogativa di riuscire a incorporare le cosiddette «arti sorelle», ossia pittura e poesia: tramite l'intonazione, la gestualità e la prossemica dell'attore, il teatro è in grado di rappresentare le emozioni nella loro completezza mentre la poesia e l'immagine lo sono parzialmente. In tale riconoscimento una funzione fondamentale riveste la Royal Academy grazie alle lezioni di Heinrich Füssli e di John Opie. Ben significative risultano, allora, le recensioni teatrali che adottano il lessico delle emozioni e parlano di «conflit of passions», «burst of feelings», «study of tempers and passions», «variety of passions», «flexibility of emotions» per valutare la raffigurazione delle passioni. Anche in questo caso una postilla: l'attenzione riservata alla performance dell'attore porterà alla nascita del cosiddetto grande attore che, nel bene e nel suo eccesso, dominerà le scene dell'Ottocento e oltre.

Rispetto al nesso musica-emozione e al suo rapportarsi con le altre arti, assai più problematica si presenta la situazione. In Francia, ad esempio, verso la fine del secolo valgono ancora le argomentazioni di Jean-Philippe Rameau il quale ritiene che, all'interno di un pezzo, sia dalla disposizione dei diversi mutamenti di tono che derivano l'espressione e il sentimento e non dalla scelta della tonalità principale. Nel 1779 Michel Paul de Chabanon reputa una chimera l'individuazione, in certi brani, di una imitazione dei fenomeni naturali: la musica può commuovere ma mettendo in atto dei propri mezzi tecnici ed endogeni. Nel 1785, BernardGermain-Ètienne de Laville, comte de La Cépède, asserisce la genericità, l'indeterminatezza delle immagini suscitate da una sinfonia, per quanto vive esse siano. Un diverso ambito geografico considerato è quello di area germanica, dove si hanno passaggi diversamente singolari per l'estetica musica- 
le: la concezione barocca secondo cui l'opera commuove ed è, quindi, bella, si rovescia nella tesi per cui l'opera è bella, dunque commuove. Una inversione di tendenza è qui testimoniata sulla celebre aria «Che farò senza Euridice», dell' Orfeo e Euridice di Gluck. E prove inerenti all'evoluzione della musica si hanno ancora per l'area germanica, dove non può mancare Mozart.

Nell'ultima parte del volume le concordanze per Sentimento e politica sono rivolte ai contesti tedesco e inglese. Con un discorso imperniato sulla diffusione e influenza delle idee, per la Germania si riflette sull'influsso esercitato da Shaftesbury nel campo dell'estetica e dell'arte per giungere ai meccanismi tipologici della sociabilità. Un influsso databile dal 1715, quando Leibniz esprime la propria sintonia con il pensiero del suo omologo inglese (Éloge critique des cuvres de Milord Shaftsbury par Mr. Le Baron de Leibnits), intorno a un nuovo platonismo e al modo di ragionare per via di interrogazioni. Influsso stemperatosi allorché si afferma una corrente antiplatonica a sua volta interrottasi con l'edizione Zweibrücker dell'opera del filosofo greco (1781-1787). E, per l'onda delle corrispondenze, si registra una simile curva cronologica per le traduzioni tedesche di Shaftesbury, attive sino a metà del secolo per poi dover aspettare gli ultimi anni Settanta e gli Ottanta per una traduzione completa delle Characteristicks. Se le interpretazioni esegetiche nell'ultimo quindicennio propendono a obiettare il paradigma platonico dell'inglese a favore di uno stoico, la verifica della fortuna di Shaftesbury privilegia in prevalenza la prima metà del Settecento. Una stima su cui, ad una attenta riconsiderazione, si deve aggiustare il tiro, proprio partendo dalla teoria shaftesburiana del moral sense e dalle sue consequenziali inclinazioni volte ad analizzare le reazioni comportamentali dell'individuo e, infine, le conseguenti ascendenze: ascendenze comprovate, ad esempio, dai tre libri di Johann Georg Zimmermann consacrati, sin dai titoli, all'antropologia della solitudine e pubblicati tra il 1756 e il 1784-1785 (Betrachtungen über die Einsamkeit, Von der Einsamkei, Über die Einsamkeit).

Per i risvolti tra sentimento e politica in Inghilterra esemplari sono due libri fra i più divulgati di Edmund Burke, editi in anni molto distanti tra loro e formalmente diversi per argomento, ovvero A Philosophical Enquiry into the Origin of Our Ideas of the Sublime and Beautiful $\left(1757,2^{\circ} \mathrm{ed} .1759\right)$ e le 
Metamorfosi dei

Lumi 10.

L'emergenza

del

sentimento
Reflections on the Revolution in France (1790) al fine di indagare sui nodi maggiormente opinabili delle interpretazioni fatte circa gli intrecci fra le «categorie estetologiche di bello e di sublime», nel loro snodarsi, e la visione politico-costituzionale nel pensiero di Burke. Si ridiscutono o si integrano, in un fitto dibattito, le deduzioni di talune letture critiche allo scopo di meglio ridefinire quale sia l'incidenza dell'emozione in ambito politico ed esaminando il caso della Rivoluzione francese il parlamentare whig Burke vede l'autorità politica ridotta a puro «strumento di coercizione».

Questo quadro sull'emergenza del sentimento è, ovviamente, parziale e, quindi, ben foriero di un moltiplicarsi e ramificarsi di correlazioni e di implicazioni che convalidano una circolazione e conoscenza delle idee, sia pur anche indiretta. Alcuni brevi esempi.

Si consideri la rilevanza europea assunta dalla natura in binomio con la solitudine. Nell'esordio del secondo Entretien sur le fils natural (Denis Diderot, 1757), il personaggio Dorval in un «endroit [...] solitaire et sauvage [...] s'était abandonné au spectacle de la nature»e, con «une voix alterée», dichiarava:

Un homme a-t-il reçu du génie? Il quitte la ville et ses abitants. Il aime, selon l'attrait de son cœur, a mêler ses pleurs au cristal d'une fontaine [...]; à fuir au fond des forets. Il aime leur horreur. Il erre. Il cherche un antre qui l'inspire.

I pochi stralci citati suggeriscono un rinvio a Vittorio Alfieri e all'incipit del suo sonetto «Tacito orror di solitaria selva», al secondo verso «Di sì dolce tristezza il cor mi bea» e alla chiusura «Sol nei deserti tacciono i miei guai» (datato 16 agosto 1786). Una legittimazione della solitudine che già il giovane Alfieri aveva manifestato, e poi testimoniato nella Vita (Epoca Terza), al tempo dei tours europei mentre attraversava le «immense selve», $i$ «lagoni crostati» del Nord Europa e i «vasti deserti d'Arragona», sintomatici, soprattutto gli ultimi, di una ricerca della solitudine non vissuta alla stregua di sottile e squisito piacere ma più intensamente come isolamento, come raccoglimento interiore, come necessario rifuggire la presenza altrui ed emblematico, e ancora ignoto, indizio di un futuro di autore:

Disgrazia mia (ma forse fortuna d'altri) che io in quel tempo non avessi nessunissimo mezzo né possibilità oramai 
di stendere in versi i miei diversi pensieri, ed affetti: ché in quelle solitudini e moto continuato avrei versato un diluvio di rime; infinite essendo le riflessioni malinconiche morali, come le imagini [sic] e terribili, e liete, e miste, e pazze che mi si andavano affacciando alla mente. Ma non possedendo io allora nessuna lingua, e non mi sognando neppure di dovere né potere scrivere nessuna cosa né in prosa né in versi, io mi contentava di ruminar fra me stesso, e di piangere alle volte dirottamente senza saper di che, e nello stesso modo di ridire.

Una ritrosia che rimanda, previo il divario dicotomico per nascita e formazione tra l'italiano e il ginevrino, a un passo della sesta delle Rêveries du promeneur solitaire del non amato (da Alfieri) Rousseau dove si evidenzia l'impulso a rifuggire «la societé civile, où tout est gêne, obligation, devoir». Un'ultima citazione dall'autobiografia alfieriana: sempre all'epoca dei viaggi si situa il ricordo di un «luoghetto graziosissimo», individuato a Marsiglia dove egli era solito bagnarsi verso sera, non scorgendo altro se non «mare e cielo»e «fra quelle immensità» trascorreva «un'ora di delizie fantasticando; e quivi avrei composte molte poesie, se io avessi saputo scrivere o in rima o in prosa in una lingua qual si fosse» (Epoca terza). L'episodio è rievocato da Chateaubriand nei suoi Mémoires d'outre-tombe: «Si les Mémoires d'Alfieri eussent été pubbliés en 1803 je n'aurais pas quitté Marseille sans visiter le rocher des bains du poète. Cet homme rude est arrivé une fois au charme de la rêverie et de l'expression».

Per l'equazione solitudine-natura, c'è da dire che Alfieri non è certo un caso isolato nel panorama italiano. Tra fine Settecento e primo Ottocento, alcuni intellettuali esaltano l'abbandonarsi alla vita solitaria coniugandola in sintonia con la natura e con la contrapposizione città-campagna: Prose e poesie campestri di Ippolito Pindemonte (1795); Piaceri della solitudine, alias il decimo dei Discorsi accademici di Domenico Cirillo (1789-1799) e si può chiudere, pur con un doveroso divario e altrettanti distinguo, con La vita solitaria di Leopardi (1821). Sono dei percorsi segnati dalla ricerca di una elitaria distinzione, di una sorta di isolamento superiore, di un raffinato e salutare rifugiarsi nella natura che si richiama, oltre a Rousseau, non soltanto ai classici (Orazio e Petrarca) ma pure a Parini e a Pope (si ravvisi di Pope la sua precoce Ode to Solitude e, per inciso, la non forse pura casualità del fatto 
Metamorfosi dei Lumi 10. L'emergenza del sentimento

che Alfieri si cimenti nella traduzione del poemetto Windsor Forest, 1790). Comunque, Alfieri è tra coloro che rielaborano nelle forme più complesse il concetto di solitudine, passando dalla giovanile e inconsapevole coscienza letteraria al consapevole impegno tragico e allora tanti personaggi vivranno destini di solitudine spietatamente necessari ed esterneranno unicamente nel soliloquio le loro passioni inconfessabili.

Nel Parere dell'Autore sulle presenti tragedie, non esita a disapprovare i soggetti privi di «contrasti di passione», come i concernenti la tragedia Maria Stuarda tanto da ritenere la protagonista «una donnuccia non mossa da passione forte nessuna; non ha carattere suo, né sublime». Alfieri non si perita di invalidare personaggi pur sublimi di per sé come Scipione (della Sofonisba) che, se pur contrassegnato dall'amicizia «caldissima per Massinissa», non è mosso da alcuna altra «calda passione». Il lemma 'passione' è una delle cifre delle figurazioni alfieriane e se ne ha conferma dallo stesso autore, leggendo un passaggio della sua Risposta a Ranieri de' Calzabigi al punto in cui, motivando le proprie scelte di stile, redige una lista delle «tragiche passioni»: «l'ira, il furore, la gelosia, l'ambizione, la libertà, la vendetta e tant'altre». Egualmente caratterizzante il lemma 'sublime': non solamente ricorre nel Parere ma si squalifica «parola-chiave del pensiero alfieriano» (vedi Arnaldo Di Benedetto) e Alessandro Verri, uno dei primi lettori e spettatori del teatro, lo usa scrivendone al fratello Pietro: «il suo talento principale è il sublime e l'orrido» (lettera del 26 settembre 1781). E ancora (lettera del 30 novembre 1782) dopo aver assistito alla recita dell'Antigone:

Questi giorni sono nel maggior entusiasmo tragico. Il Conte Alfieri, di cui si parlava molto da vari anni, ora finalmente ha rappresentato una sua tragedia a questo Palazzo di Spagna, la quale fa strepito ed è generalmente ammirata [...]; l'interesse è sostenuto e l'effetto sommo. L'elocuzione è bellissima, i concetti sono sublimi.

Certamente per l'emergere del sentimento un fecondo terreno di sviluppo e di osservazione è offerto dalla pratica drammaturgica quando si attua una svolta nell'iter storicostrutturale della tragedia (e dei generi teatrali in generale) e compaiono delle nuove sensibilità volte a favorire le affezioni e i valori personali dei vincoli sentimentali, in particolare 
per quanto attiene ai personaggi femminili, Se Alfieri ne è stato - fors'anche malgré lui - un anticipatore per alcune figurazioni, nel passaggio tra i due secoli queste dinamiche sono riscontrabili in tragedie di autori ben conosciuti.

Pochi nomi di autori e di protagoniste, e relativi testi. Francesco Saverio Salfi con Sgomburga della Virginia bresciana (1797); Giovanni Pindemonte con Eufrasia dell' Orso Ipato (1797) e Vincenzo Monti con Licinia del Caio Gracco (1802). Sono eroine che piangono, che svengono e che spesso si abbandonano a una più famigliare e privata mozione degli affetti.

E proseguendo il percorso negli anni ci si avvierà verso una sensiblerie che tenderà a ideare una donna ormai disgiunta dalla esemplarità di parti sublimi e predisposta a scendere a più modesti patti con la realtà quotidiana: ma qui si aprirebbero tutt'altri sipari.

\section{Bibliografia}

Arnaldo Di Benedetto, Alfieri e le passioni, in Id., Le passioni e il limite. Un'interpretazione di Vittorio Alfieri, Napoli, Liguori 1994, nuova edizione riveduta e accresciuta (in particolare le pp. 3848).

Marco Cerruti, Solitari e solitudini, in Id., Il piacere di pensare. Solitudini. Rare amicizie. Corrispondenze intorno al 1800, Modena, Mucchi Editore 2000.

Silvia Contarini, Alfieri e il sublime dell'origine, in Metamorfosi dei Lumi. Esperienze dell'io e creazione letteraria tra Sette e Ottocento, a cura di Simone Carpentari Messina, Alessandria, Edizioni dell'Orso 2000.

Marco Menin, Il fascino dell'emozione, Bologna, Il Mulino 2019.

Marco Menin, La filosofia delle lacrime. Il pianto nella cultura francese da Cartesio a Sade, Bologna, Il Mulino 2019.

Paola Trivero, Eroine solitarie e donne sensibili, in Metamorfosi dei Lumi. Esperienze dell'io e creazione letteraria, cit.

Paola Trivero, Solitudini alfieriane, in Ead., Percorsi alfieriani, Alessandria, Edizioni dell'Orso, 2014. 

Parte prima

Sentimento e morale

aA 

Metamorfosi dei

Lumi 10.

L'emergenza del

sentimento
La riflessività ed oggettività della morale nella ricostruzione sentimentalista di David Hume

Maurizio Balistreri

Secondo Hume per spiegare il fenomeno della moralità bisogna partire dalla scienza dell'uomo: «Possiamo così fondamentalmente indicare - scrive Lecaldano - il punto di partenza della ricerca di Hume: superare i fantasiosi schemi di virtù e di felicità sostenuti dalla filosofia morale del passato e in modo più ravvicinato dall'etica cristiana predicata nella Scozia del suo tempo, impostando una ricerca empirica sulla natura umana» ${ }^{1}$. La scienza dell'uomo, cioè, deve essere radicata nell'esperienza e nell'osservazione: inoltre, bisogna procedere facendo valere la metodologia newtoniana ${ }^{2}$. Il tentativo di applicare la metodologia di Newton alla realtà non significa per Hume soltanto la ricerca di pochissimi principi esplicativi - il più possibile universali - il rifiuto della metafisica e di qualsiasi ipotesi fittizia relativa alle essenze o qualità occulte, ma anche rendere conto soltanto di ciò che è osservabile ${ }^{3}$.

1. E. Lecaldano, Hume e la nascita dell'etica contemporanea, Laterza, Roma-Bari 1992, p. 7.

2. Cfr. $i v i$, pp. 35-36.

3. Cfr. $i v i$, p. 36 . 
Metamorfosi dei

Lumi 10.

L'emergenza

del

sentimento

Per Hume l'essere umano, pur muovendosi in una condizione di scarsità è motivato non soltanto da un interesse egoistico ma anche dalla benevolenza e dalla generosità. Non è la benevolenza universale che suggerivano Shaftesbury ${ }^{4}$ e Hutcheson ${ }^{5}$, quanto piuttosto un impasto di interesse egoistico e di benevolenza limitata. L'antropologia di Hume, comunque, resta lontana dalla concezione hobbesiana. Secondo Hobbes, gli altri diventano oggetto del mio interesse soltanto perché possono danneggiarmi o uccidermi, negli altri casi mi sono del tutto indifferenti ${ }^{6}$. Secondo Hume, invece, la tragedia più grande che possa capitarci è quella di essere disprezzati dai nostri simili e di essere condannati ad una vita di solitudine. Il diverso quadro antropologico appare chiaro se consideriamo la passione dell'orgoglio: Hobbes assimila l'orgoglio alla passione della gloria ovvero al sentimento che proviamo quando la nostra forza e il nostro potere vengono riconosciuti; in Hume, invece, l'orgoglio non ha più per oggetto soltanto le qualità dell'io, perché si può provare anche nei confronti delle persone alle quali siamo legati. Le passioni dell'orgoglio e dell'umiltà, inoltre, nascono in noi anche in relazione al giudizio che le altre persone si fanno del nostro comportamento e carattere: la loro approvazione ci inorgoglisce, ci vergogniamo invece per la loro disapprovazione $^{7}$. Per questo, per Hume, l'orgoglio non è solo

4. A.A. Cooper Shaftesbury, Sensus Communis, an Essay on the Freedom of Wit and Humour in a Letter to a Friend, in Id., Characteristics of Men, Manners, Opinions, Times, a cura di L.E. Klein, Cambridge University Press, Cambridge 1999, pp. 29-69; trad. it. Sensus Communis. Saggio sulla libertà di spirito e di umorismo, a cura di A. Taraborrelli, Utet, Torino 2007.

5. F. Hutcheson, An Inquiry into the Original of our Ideas of Beauty and Virtue, in Id., Collected Works of Francis Hutcheson, a cura di B. Fabian, 7 voll., Olms, Hildesheim 1971, vol. I; trad. it. Ricerca sull'origine delle nostre idee di bellezza e di virtù, a cura di A. Lupoli, Baldini \& Castoldi, Milano 2000. F. Hutcheson, An Essay on the Nature and Conduct of the Passions and Affections, with Illustrations on the Moral Sense, vol. II; trad. it. Saggio sulla natura e condotta delle passioni, a cura di L. Turco, Clueb, Bologna 1997.

6. T. Hobbes, Leviathan, a cura di R. Tuck, Cambridge University Press, Cambridge 1991; trad. it. Leviatano, a cura di A. Pacchi, Laterza, Roma-Bari 2000.

7. Su questo punto ritorna anche Smith: «La giurisdizione dell'uomo interiore si fonda del tutto sul desiderio dell'essere degno di lode e nell'avversione per l'essere degno di biasimo, nel desiderio di possedere quelle qualità, e di compiere quelle azioni che amiamo e ammiriamo negli altri, e nel timore di possedere quelle qualità e di compiere quelle azioni che negli altri odiamo e disprezziamo». A. Smith, The Theory of Moral Sentiments, a cura di D.D. Raphael e A.L. Macfie, in The Glasgow Edition of the Works and Correspondence of Adam Smith, vol. I, Clarendon 
La riflessività ed oggettività della morale nella ricostruzione sentimentalista di David Hume potenziale fonte di guerra di tutti contro tutti ma è anche una componente necessaria del processo di civilizzazione. In Hobbes, cioè, la natura umana riparte sempre da zero e non vi è nessun'abitudine che va a incidere e modificare le condizioni di primigenia insicurezza ${ }^{8}$. Per Hume, invece, attraverso l'orgoglio e l'umiltà noi impariamo a misurare l'appropriatezza delle nostre azioni ed a correggere il nostro comportamento, perché non vogliamo ricevere il disprezzo e la disapprovazione dell'umanità ${ }^{9}$ Hume, poi, propone un radicale rovesciamento rispetto all'antropologia intellettualistica, repressiva ed ascetica che veniva espressa nella religione del suo tempo. L'essere umano che viene tratteggiato da Hume, cioè, è «prevalentemente fatto di istinti, abitudini, passioni e scarsissimi sono il ruolo e la portata della ragione» ${ }^{10}$. Nella prospettiva humeana la ragione non soltanto è, ma deve rimanere schiava delle passioni, in quanto soltanto le passioni possono salvarci dallo scetticismo. Secondo Hume, del resto, la ragione ci porta a dubitare di qualsiasi certezza: tuttavia, nemmeno l'argomento razionale più elaborato e accurato è capace di spegnere la vivacità che accompagna le nostre idee del mondo e dell'identità dell'io. Quando siamo calati negli 'affari comuni della vita' non c'è spazio per lo scetticismo perché le passioni impediscono che il delirio filosofico diventi una malattia ${ }^{11}$.

Anche il fenomeno della morale, poi, non dipende dalla ragione, ma dai sentimenti che proviamo nei confronti delle azioni e delle qualità dei caratteri che valutiamo ${ }^{12}$. Chiamiamo virtuose, del resto, quelle condotte e quel carattere che suscitano in noi piacere, mentre chiamiamo viziose quelle che invece ci causano dolore:

Press, Oxford 1976; trad. it. Teoria dei sentimenti morali, traduzione di S. Di Pietro, BUR, Milano 2001, p. 285.

8. E. Lecaldano, Hume e la nascita dell'etica contemporanea, cit., p. 57.

9. L. Greco, On Pride, «Humana.Mente Journal of Philosophical Studies», 35 (2019), pp. 101-123.

10. E. Lecaldano, Hume e la nascita dell'etica contemporanea, cit., p. 79.

11. Cfr. D.C. Ainslie, Hume's True Scepticism, Oxford University Press, Oxford 2017; J. Wright, Hume's Skeptical Realism, in P. Russell (a cura di), The Oxford Handbook of Hume, Oxford University Press, Oxford-New York 2016, pp. 60-81.

12. J. Taylor, Moral Sentiment and the Sources of Moral Identity, in C. Bagnoli (a cura di), Morality and the Emotions, Oxford University Press, Oxford 2011, pp. 257-274. 
Metamorfosi

dei

Lumi 10.

L'emergenza

del

sentimento

Le distinzioni morali - scrive Hume - dipendono completamente da certi particolari sentimenti di dolore e di piacere, e [...] qualsiasi qualità mentale, nostra o altrui, capace di darci soddisfazione al solo vederla o pensarla, è decisamente virtuosa; come invece viziosa è qualsiasi cosa, di questa natura, che ci dia dolore ${ }^{13}$.

Il dolore o il piacere che sorge in noi di fronte o alla vista di un'azione oppure di un carattere, ne costituisce il vizio o la virtù e genera la nostra approvazione o il nostro biasimo, «che altro non è - dice Hume - se non un amore o un odio più debole e impercettibile» ${ }^{14}$. Hume non vuole avvicinarsi alla morale come un pittore, ma piuttosto come un anatomista: la sua scienza dell'uomo, cioè, non si propone di fare risaltare la grazia e la bellezza del suo oggetto ma di scoprirne i più profondi principi ed origini. La sua ricostruzione della morale può essere letta come un tentativo di superare lo scetticismo morale promosso dalla filosofia hobbesiana con un fondamento alternativo rispetto a quello di tipo razionale o garantito dall'ordine divino. Prima di Hume, altri autori avevano cercato di spiegare l'etica con i sentimenti. Già Shaftesbury aveva voluto mostrare non soltanto che esiste un senso naturale del giusto e dell'ingiusto ma che esistono anche sentimenti non egoistici che mettono gli esseri umani in relazione con l'interesse della specie e della comune natura. Noi siamo esseri socievoli: «Come mangiare e bere sono naturali per l'uomo, non diversamente naturale è l'istinto di socievolezza che porta gli uomini ad associarsi» ${ }^{15}$. Secondo Shaftesbury, poi, non soltanto la socievolezza umana è qualcosa di naturale, ma c'è anche sentimento di appartenenza alla comunità che proviamo quando percepiamo che le nostre azioni sono in armonia con il tutto (sense of fellowship). In Hutcheson, poi, è possibile conoscere che cosa è giusto e cosa è ingiusto perché abbiamo un 'senso morale' che è una sorta di senso aggiunto le cui percezioni, come nel caso degli altri sensi, sono valutabili nei termini di verità e falsi-

13. D. Hume, A Treatise of Human Nature, a cura di L.A. Selby-Bigge, P.H. Nidditch, Clarendon Press, Oxford 1978; trad. it. in Opere filosofiche, a cura di E. Lecaldano, Laterza, Roma-Bari 1987, p. 608.

14. D. Hume, Trattato sulla natura umana, cit., p. 648.

15. E. Lecaldano, Lilluminismo inglese, Loescher, Torino 1985, p. 26. 
La riflessività ed oggettività della morale nella ricostruzione sentimentalista di David Hume $\operatorname{tà}^{16}$. Secondo Hume, invece, il senso morale non è affatto in grado di fornire conclusioni effettivamente reali e oggettive simili a quelle trasmesse dalle impressioni dei sensi. Il vizio e la virtù, il bene e il male, infatti, sono più simili alle qualità secondarie: queste distinzioni sono impressioni di riflessione ovvero non sono impressioni immediate ma sono causate da altre impressioni o idee che colpiscono la mente. Nel primo libro del Trattato, Hume distingue le impressioni dalle idee e poi distingue le impressioni in impressioni di sensazione e impressioni di riflessione: le prime nascono da cause ignote, le seconde dalle nostre idee ricevute attraverso i sensi. Ad esempio, le idee di piacere e di dolore, quando tornano alla mente, producono le nuove impressioni di desiderio e di avversione, di speranza e di timore. Ma anche i sentimenti morali sono impressioni di riflessione, in quanto sono risposte soggettive ai motivi, alle condizioni di giustificazione ed alle conseguenze delle azioni.

Il problema, - afferma Hume - ora, è quello di vedere quale sia la natura di queste impressioni [...]. È impossibile qui nutrire molte esitazioni, ma dovremmo affermare che l'impressione che sorge dalla virtù è gradevole e che quella che deriva dal vizio è sgradevole; in qualsiasi momento l'esperienza può convincerci di questo ${ }^{17}$.

Non c'è, cioè, spettacolo tanto amabile e bello quanto quello di un'azione nobile e generosa, né uno che susciti in noi più avversione di un'azione crudele e sleale. Anche solo una rappresentazione teatrale o un romanzo - conclude Hume - possono darci esempi del piacere che la virtù ci procura e del dolore che sorge dal vizio ${ }^{18}$.

All'interno di questa ricostruzione della moralità, comunque, un ruolo importante viene giocato dalla simpatia ${ }^{19}$, in quanto per Hume noi esprimiamo approvazione o disapprovazione perché entriamo in contatto con i piaceri e dolo-

16. A. Vaccari, Le etiche della virtù. La riflessione contemporanea a partire da Hume, Le Lettere, Firenze 2012, pp. 90-91; P.J. Kail, Hutcheson's Moral Sense: Skepticism, Realism, and Secondary Qualities, «History of Philosophy Quarterly», 19 (2001), pp. 5777 .

17. D. Hume, Trattato sulla natura umana, cit., pp. 497-498.

18. Ibid.

19. R. Vitz, The Nature and Functions of Sympathy in Hume's Philosophy, in P. Russell (a cura di), The Oxford Handbook of Hume, cit., pp. 312-332. 
Metamorfosi dei Lumi 10. L'emergenza del sentimento

ri rilevanti ${ }^{20}$. Anche in Hobbes troviamo il riconoscimento dell'influsso di una sorta di contagio emotivo tra gli esseri umani che trasporta gli spiriti animali facendo nascere passioni quali l'orgoglio o la vergogna e provocando l'arrossire del volto oppure il riso. La trasmissione delle emozioni e delle passioni è però immediata e non c'è alcuna necessità di riconoscere qual è la passione che viene provata dall'altra persona. Inoltre, questo contagio non comporta una partecipazione con le passioni dell'altro, in quanto, ad esempio, davanti alle sofferenze degli altri posso provare compassione semplicemente perché posso immaginare di vivere io stesso quella condizione. In Hume invece la simpatia non soltanto perde anche le sue strette connessioni con la compassione e le altre inclinazioni che comportano un effettivo interessamento pratico per gli altri, ma non è nemmeno un mero contagio emozionale. È piuttosto una pratica sociale che richiede educazione e che si può raffinare nel tempo vivendo insieme in società con altre persone ed imparando a coordinare con gli altri i propri comportamenti senza bisogno di una coercizione esterna ${ }^{21}$.

La simpatia si attiva attraverso i segni esteriori, espressi nel contegno e nella conversazione:

È certo evidente che quando proviamo simpatia per le passioni e i sentimenti degli altri, questi moti dell'animo appaiono inizialmente nella nostra mente come mere idee, e vengono pensati come appartenenti a un'altra persona, non diversamente da come concepiamo qualsiasi altro dato di fatto. È altresì evidente - scrive Hume - che le idee delle affezioni altrui si convertono nelle impressioni stesse che rappresentano e che le passioni sorgono in conformità alle impressioni che ce ne formiamo ${ }^{22}$.

L'idea della passione di un'altra persona acquista, poi, vivacità attraverso l'impressione di noi stessi, che ci è sempre intimamente presente. "[L']idea, o piuttosto l'impressione di noi stessi, - afferma Hume - ci è sempre intimamente presente, $[\ldots]$ la nostra coscienza ci dà una rappresentazione della nostra persona tanto viva da non poter immaginare che

20. E. Lecaldano, Hume e la nascita dell'etica contemporanea, cit., p. 82.

21. A. Vaccari, Passions and Sympathy in Hume's Philosophy, in A. Coventry e A. Sager (a cura di), Humean Mind, Routledge, London - New York 2018, pp. 195-208.

22. D. Hume, Trattato sulla natura umana, cit., p. 335. 
La riflessività ed oggettività della morale nella ricostruzione sentimentalista di David Hume qualcosa possa in questo superarla. Quindi, qualsiasi oggetto sia in relazione con noi deve essere concepito con un'analoga vivacità di rappresentazione [...] e sebbene questa relazione non dovrebbe essere così forte come quella di causalità, deve pur tuttavia avere una notevole influenza» ${ }^{23}$.

Tuttavia, la simpatia può anche essere messa in moto non soltanto dall'osservazione degli effetti di una passione, ma anche dalle sue cause abituali. Ad esempio, dalla presenza di strumenti chirurgici accanto a una persona distesa su un lettino in attesa di un'operazione, possiamo immaginarci i suoi sentimenti: ad esempio, la sua paura e le sue ansie per un intervento al quale potrebbe anche non sopravvivere. La simpatia, cioè, può sorgere anche soltanto dalla vista della situazione che generalmente la suscita: non è necessaria la percezione della passione ${ }^{24}$.

L'analogia con la relazione causa-effetto è evidente: per le leggi di associazione, alla vista o al pensiero di un oggetto, la mia mente passa ad un'altra idea a cui la mia percezione conferisce parte della sua vivacità, facendo nascere la credenza. Affinché questo avvenga, però, dobbiamo osservare una connessione ripetuta tra due diversi oggetti, perché altrimenti la mente non passa al secondo oggetto. Tuttavia, non esiste alcuna necessità oggettiva che all'apparire del primo fenomeno debba seguire quello che solitamente l'accompagna: siamo noi che al percepire di un oggetto passiamo subito al secondo e crediamo che il secondo debba manifestarsi. "Quando un'impressione ci si presenta, non soltanto trasporta la mente a quelle idee che sono in relazione con essa, ma comunica loro anche una parte della sua forza e vivacità» ${ }^{25}$. Non è pertanto la ragione a dimostrare o stabilire la connessione: «Quando, dunque, la mente passa dall'idea o impressione d'un oggetto all'idea o credenza d'un altro, non è determinata dalla ragione, ma da certi principi che associano tra loro le idee di questi oggetti e le uniscono

23. Ivi, p. 333.

24. Su questo è d'accordo anche Adam Smith: «Arrossiamo per la sfrontatezza e la rozzezza di un altro, nonostante egli stesso sembri non rendersi conto dell'inappropriatezza del suo comportamento, perché non possiamo evitare di sentire la vergogna di cui ci saremmo coperti se fossimo stati noi a comportarci in una maniera così assurda» (Teoria dei sentimenti morali, cit., p. 86).

25. D. Hume, Trattato sulla natura umana, cit., p. 112. 
Metamorfosi dei

Lumi 10.

L'emergenza

del

sentimento nell'immaginazione ${ }^{26}$. La chiave è sempre il passaggio di vivacità: «Onde accade che, una volta che la mente sia ravvivata da un'impressione presente, seguita a formare idee più vivaci degli oggetti relativi per un naturale passaggio della sua disposizione dall'una agli altri» ${ }^{27}$.

2. Il carattere riflessivo dell'etica ed il punto di vista fermo e generale

La nostra simpatia è limitata, in quanto simpatizziamo di più con chi è vicino nel tempo e nello spazio: i sentimenti degli altri, cioè, - scrive Hume - hanno scarsa influenza, quando ci sono molto lontani, e c'è bisogno della relazione di contiguità per comunicarsi pienamente. A volte, però, anche le relazioni di parentela possono produrre lo stesso effetto, così come la familiarità che agisce allo stesso modo dell'educazione e delle abitudini. "Tutte queste relazioni, se unite insieme, - afferma Hume - fanno passare l'impressione o la consapevolezza della nostra persona all'idea dei sentimenti o passioni degli altri, facendoceli concepire nel modo più forte e più vivo» ${ }^{28}$.

Tuttavia, malgrado la parzialità della simpatia, i nostri giudizi morali non cambiano a seconda della nostra vicinanza o lontananza da situazione che giudichiamo. L'oggettività della morale, secondo Hume, è assicurata dal fatto che abbiamo imparato ad assumere un punto di vista indipendente dalla nostra posizione presente. Non posso provare per le virtù di una persona che visse in Grecia duemila anni fa lo stesso vivo piacere che provo davanti alle virtù di un amico che conosco intimamente: comunque, sono in grado di apprezzare allo stesso modo il loro carattere perché sono in grado di prendere le distanze dal mio punto di vista particolare. Del resto, spiega Hume, quando valutiamo le persone basandoci solo sulla tendenza del loro carattere a essere di vantaggio per noi o per i nostri amici, ci scontriamo con così numerose contraddizioni con i nostri sentimenti nella società e nella conversazione, che sentiamo la necessità di un criterio di merito e di demerito più stabile ${ }^{29}$ :

26. Ivi, p. 105 .

27. Ivi, p. 113.

28. Ivi, p. 334.

29. Cfr. $i v i$, p. 617. 
La riflessività ed oggettività della morale nella ricostruzione sentimentalista di David Hume
La nostra situazione, sia rispetto alle persone sia rispetto alle cose, è continuamente fluttuante, e un uomo che ci sia lontano può diventare in breve tempo un conoscente intimo. Inoltre, ogni singolo uomo ha una sua particolare posizione rispetto agli altri; e ci sarebbe impossibile riuscire mai ragionevolmente a conversare insieme, se ognuno di noi dovesse considerare caratteri e persone unicamente da come ci appaiono dal nostro particolare punto di vista. Quindi, per prevenire queste continue contraddizioni e raggiungere una maggiore stabilità nei nostri giudizi sulle cose, fissiamo certi punti di vista fermi e generali, e sempre, nei nostri pensieri, ci riferiamo a essi, quale che sia la nostra situazione attuale [...]. L'esperienza - conclude Hume - ci insegna ben presto questo metodo per correggere i nostri sentimenti o, almeno, per correggere il nostro linguaggio quando i sentimenti sono più ostinati e resistenti ${ }^{30}$.

La soluzione che appare più oggettiva per misurare le "qualità" di una persona e il suo "carattere" è la simpatia con coloro che hanno dei rapporti con la persona in questione ${ }^{31}$. In questo modo, infatti, l'oggetto della valutazione diventa lo stesso per tutti e anche persone con punti di vista ed interessi diversi possono trovarsi d'accordo. Inoltre, possiamo anche credere che una persona abbia le migliori intenzioni, ma è soltanto nella sua cerchia ristretta che emergono i segni delle sue qualità, in quanto la maggior parte delle nostre azioni produce conseguenze soprattutto sulle persone con le quali viviamo o con le quali abbiamo una qualche relazione. Simpatizzando, pertanto, con chi subisce maggiormente gli effetti del comportamento della persona il cui carattere stiamo prendendo in considerazione, correremo meno il rischio di sbagliare e di attribuirgli meriti o demeriti che non possiede. È vero che quando fissiamo la nostra attenzione sulle persone lontane o estranee, la simpatia non è così viva come quando in gioco è il nostro interesse o quello dei nostri amici; né essa può avere la stessa influenza sul nostro amore ed odio. Tuttavia, essa conserva la capacità di guidare i nostri giudizi e le nostre opinioni: per questa ragione, noi possiamo biasimare «tanto un'azione malvagia di cui ci parla la storia, quanto una compiuta l'altro giorno dal nostro vicino: e questo significa 
Metamorfosi dei Lumi 10. L'emergenza del sentimento

che, riflettendoci su, sappiamo che la prima azione susciterebbe sentimenti di disapprovazione non meno forti della seconda, se fosse posta nella stessa posizione ${ }^{32}$.

Per Hume, pertanto, la morale è tutt'altro che naturale e originaria, come invece pensavano Shaftesbury e Hutcheson: è, invece, un prodotto della civilizzazione, frutto di correzioni e invenzioni operate a partire dalle nostre esperienze ${ }^{33}$. Questa è la ricostruzione della morale che troviamo già in Hobbes e Locke ${ }^{34}$ : in Hume, però, dietro questo fenomeno non si nasconde più la ragione che raccomanda di uscire dallo stato di natura o che distingue i comportamenti, raccogliendoli sotto idee di modo misto che non hanno alcuna corrispondenza nella realtà. Le nostre distinzioni morali hanno origine dal piacere e dal dolore che proviamo: ma queste reazioni non sono qualcosa di viscerale e non vanno confuse con passioni come l'odio, l'amore o la paura sono invece sentimenti di secondo livello ${ }^{35}$, perché la vita in società insegna ad adottare un punto di vista fermo e generale. Come, poi, affermerà Adam Smith che condivide con Hume la ricostruzione sentimentalista della morale, è un'operazione che l'abitudine e l'esperienza ci hanno insegnato a compiere così facilmente e velocemente che a mala pena ci accorgiamo di guardare le cose con imparzialità, con gli occhi di una terza persona ${ }^{36}$ :

È la ragione, il principio, la coscienza, l'abitante dell'animo, l'uomo interiore, il grande giudice e arbitro della nostra condotta. È lui che, ogni volta che stiamo per colpire la felicità altrui, ci grida, con una voce capace di stordire le nostre passioni più presuntuose, che noi non siamo altro che uno dei tanti, sotto nessun riguardo migliore di qualsiasi altro, e che quando, in modo così vergognoso e cieco, preferiamo noi agli altri, diventiamo oggetti appropriati di risentimento, avversione e disprezzo ${ }^{37}$.

32. Ivi, p. 617.

33. Cfr. J. Taylor, Reflecting Subjects: Passion, Sympathy, and Society in Hume's Philosophy, Oxford University Press, Oxford 2015.

34. Cfr. J. Locke, An Essay concerning Human Understanding, a cura di P. H. Nidditch, Oxford University Press, Oxford 1979; trad. it. Saggio sull'intelligenza umana, Laterza, Roma-Bari 1999.

35. Cfr. E. Lecaldano, Prima lezione di filosofia morale, Laterza, Roma-Bari 2010, pp. 55-56.

36. Cfr. A. Smith, Teoria dei sentimenti morali, cit., p. 292.

37. A. Smith, Teoria dei sentimenti morali, cit., p. 295. 
La riflessività ed oggettività della morale nella ricostruzione sentimentalista di David Hume

A differenza di Smith, Hume non propone un modello di comportamento virtuoso basato sull'ascetismo: al contrario, mostra i pericoli di un'etica della costrizione e della rinuncia. Tuttavia, anche per Hume la vita in società produce una seconda natura ${ }^{38}$ che coincide con la capacità di adottare un punto di vista generale e comune ${ }^{39}$. Con l'acquisizione di questa prospettiva c'è sempre la stessa persona: ad ogni modo, quando mi sforzo di esaminare la condotta degli altri o il mio stesso comportamento, l'io esaminatore è un personaggio diverso rispetto alla persona che agisce, perché guarda le cose da spettatore, cioè a distanza e con gli occhi degli "altri". Hume non approfondisce i contrasti che possono sorgere tra lo spettatore imparziale e la persona reale, ma possiamo ricostruirli attraverso la riflessione di Smith. L'uomo interiore può rassicurarci e correggere il giudizio falso delle altre persone, ma, quando non ci comportiamo bene, è pronto a disapprovare la nostra condotta. Per questo, a volte proviamo ad ingannarlo, sforzandoci di guardare le cose non attraverso gli occhi degli altri, ma come ci piacerebbe che le altre persone le guardassero. Quest'autoinganno, poi, può essere rafforzato dall'ammirazione delle altre persone, che siamo in grado di conquistarci attraverso la menzogna e l'apparenza. Inoltre, lo spettatore imparziale possiede nei confronti di qualsiasi persona un'autorevolezza difficile da contrastare perché è la voce del punto di vista generale. Tuttavia, anche per lo spettatore imparziale non è facile conservare la propria fermezza e la propria capacità di giudizio davanti al biasimo delle altre persone, la cui violenza può paralizzare la convinzione soggettiva dell'essere degni di lode:

Osiamo appena assolverci, - scrive Smith - quando tutti i nostri fratelli sembrano vistosamente condannarci. Sembra che l'immaginato spettatore imparziale della nostra condotta dia la sua opinione in nostro favore con timore ed esitazione, mentre quella degli spettatori reali, quella di tutti coloro con i cui occhi e dalla cui posizione egli cerca di considerare

38. Cfr. $i v i$, p. 257.

39. Cfr. L. Greco, Simpatia ed etica: in difesa della prospettiva humeana, «I castelli di Yale online», 4 (2016), n. 2, pp. 1-14; Id., L'io morale. David Hume e l'etica contemporanea, Liguori, Napoli 2008, pp. 146-161. 
Metamorfosi dei Lumi 10. L'emergenza del sentimento la nostra condotta, è unanimemente e violentemente contro di noi ${ }^{40}$.

Anche se, però, il rapporto tra l'uomo interiore e la persona reale è complesso, il punto di vista fermo e generale o comune, incarnato dallo spettatore imparziale, è la prova migliore del carattere riflessivo dell'etica sentimentalista humeana. A differenza, cioè, di quanto affermato dalle critiche di matrice kantiana, si può concludere, seguendo Vaccari, che il sentimentalismo humeano «sostiene una concezione dell'etica secondo la quale le virtù degli agenti sono modellate e sostenute dalle loro capacità riflessive e sono quindi qualcosa di cui essi sono responsabili» ${ }^{41}$.

\section{Il carattere e le sue qualità come oggetto della morale}

Secondo Hume, al centro della considerazione morale non sono le azioni, ma il carattere ${ }^{42}$. Le nostre azioni sono importanti soltanto perché sono il segno di un carattere: se non sono espressione di un carattere il loro significato svanisce, in quanto o sono semplicemente casuali oppure sono soltanto il risultato di un errore. Secondo Hume, del resto, è evidente che «le nostre azioni possiedono una unione costante con i nostri motivi, i nostri caratteri e con le circostanze in cui ci troviamo [...]» ${ }^{43}$. Non può esserci, infatti, - scrive Hume - nessuna unione più costante e certa di quella che sussiste tra alcune azioni e certi motivi e qualità dell'agente considerato $^{44}$. Per questa ragione, nelle nostre considerazioni morali, la nostra attenzione va alle disposizioni del carattere che acquistano visibilità attraverso le azioni:

Un'azione è virtuosa o viziosa se è segno di una qualità o d'un carattere. Essa deve dipendere da durevoli principi della mente che si estendono a tutta la condotta e fanno parte del carattere personale. Le azioni, di per sé, se non derivano da un principio costante, non hanno alcuna influenza sull'amore o sull'odio, sull'orgoglio o sull'umiltà, e di conseguenza non sono mai prese in considerazione in mo-

40. Cfr. $i v i$, p. 286.

41. A. Vaccari, Le etiche della virtù, Le Lettere, Firenze 2012, p. 58.

42. A.C. Baier, A Progress of Sentiments. Reflections on Hume's Treatise, Harvard University Press, Cambridge (Massachusetts) 1991.

43. D. Hume, Trattato sulla natura umana, cit., p. 421.

44. Cfr. ivi, p. 424. 
La riflessività ed oggettività della morale nella ricostruzione sentimentalista di David Hume rale. [...] Nelle nostre ricerche sull'origine della morale non dobbiamo mai prendere in considerazione un'azione isolata, ma solo la qualità o il carattere da cui tale azione deriva. Soltanto questa qualità o questo carattere sono abbastanza durevoli in una persona per influenzare i nostri sentimenti nei suoi confronti. Le azioni, è pur vero, molto meglio delle parole, o anche dei desideri e dei sentimenti, sono indici di un carattere, ma - afferma Hume - è soltanto nella misura in cui esse sono appunto indici di un carattere, che sono accompagnate da amore e da odio, da lode o da biasimo ${ }^{45}$.

Secondo Hume, le disposizioni del carattere che approviamo sono quelle che risultano essere utili al soggetto o agli altri oppure gradevoli al soggetto o agli altri. Ogni qualità della persona che dia piacere al solo vederla viene denominata infatti virtuosa, mentre viene chiamato vizioso ogni aspetto del carattere che produce dolore ${ }^{46}$ :

Ora, quando giudichiamo il carattere di qualcuno, il solo interesse o piacere che appare identico a ogni osservatore è quello della persona stessa il cui carattere viene preso in esame o quello delle persone che sono in rapporto con lei. $\mathrm{E}$ sebbene questi interessi e piaceri ci tocchino più debolmente dei nostri, pur tuttavia, dal momento che sono più costanti e universali essi riescono a controbilanciarli in pratica e sono i soli a essere ammessi nelle nostre speculazioni come criteri della virtù e della morale ${ }^{47}$.

Secondo Hume, comunque, possiamo distinguere tra virtù naturali e virtù artificiali: le virtù naturali sono quelle che suscitano per natura particolari reazioni e che producono sempre azioni utili o gradevoli; quelle artificiali nascono a seguito dell'affermarsi di una convenzione e non producono sempre, in ogni situazione immaginabile, azioni utili o gradevoli, ma producono, in generale, vantaggi importanti. Riguardo, inoltre, alle virtù naturali, si possono distinguere quelle che permettono alle persone di svolgere il loro ruolo nella società e quelle che, invece, sono vantaggiose a chi le possiede, mettendolo in grado di servire il suo interesse personale. Per Hume, del resto, la prudenza, la temperanza, la 
Metamorfosi dei Lumi 10.

L'emergenza del sentimento frugalità, l'operosità, l'assiduità, l'iniziativa e la destrezza non valgono meno della generosità e dell'umanità ${ }^{48}$ :

Prendiamo un uomo le cui qualità sociali non siano particolarmente manchevoli, ma che si distingua principalmente per la sua abilità negli affari, con la quale è riuscito a districarsi nelle maggiori difficoltà e a risolvere le questioni più delicate con singolare destrezza e prudenza. Trovo - conclude Hume - che immediatamente nasce in me una stima per lui: la sua compagnia è per me fonte di soddisfazione, e prima ancora di conoscerlo meglio preferirei rendermi utile a lui piuttosto che a un altro il cui carattere sia uguale in tutto il resto, ma manchevole in questo aspetto. In un caso simile, le qualità di cui mi compiaccio vengono considerate utili a chi le possiede e inclini a favorire il suo interesse e la sua soddisfazione ${ }^{49}$.

Dovremmo considerare, poi, che alcune qualità derivano il loro merito dall'essere immediatamente gradevoli alle altre persone oppure gradevoli alle persone che le possiedono ${ }^{50}$. Anche se, poi, si ritiene che a esse non sia collegato alcun merito o valore morale, sono virtù anche le attitudini naturali, come, ad esempio, le doti fisiche, il buon senso, il discernimento, la prudenza e la discrezione, l'arguzia, l'eloquenza e la pulizia. Non ha alcun senso, infatti, distinguere tra attitudini naturali e virtù morali: non soltanto entrambe sono qualità del carattere; ma entrambe producono anche piacere e hanno una uguale tendenza naturale a ricevere l'amore e la stima dell'umanità ${ }^{51}$ : inoltre, «danno nuovo lustro alle altre virtù e $[. .$.$] chi le possiede ha molto più diritto alla$ nostra benevolenza e ai nostri favori di chi ne è totalmente privo» ${ }^{52}$. A questo si aggiunga che molte di quelle qualità che i moralisti chiamano virtù, sono altrettanto involontarie e necessarie delle qualità del giudizio e dell'immaginazione. Non c'è, cioè, alcun merito nelle qualità che fanno grande un personaggio e lo stesso si può dire per le qualità che invece rendono un altro biasimevole: del resto, tanto più certe
48. Cfr. $i v i$, p. 620.
49. Ivi, pp. 621-622.
50. Ivi, p. 623.
51. Cfr. ivi, pp. 640-641.
52. Ivi, p. 641. 
La riflessività ed oggettività della morale nella ricostruzione sentimentalista di David Hume qualità sono viziose, tanto meno sono volontarie ${ }^{53}$. Anche la bontà e benevolenza, infine, sono virtù naturali in quanto sono utili alle altre persone: tuttavia, dobbiamo tener conto che la generosità umana è molto limitata e che raramente si estende al di là degli amici e della famiglia o del paese natale. Da una persona buona, non dobbiamo, cioè, aspettarci comportamenti impossibili, ma limitare il nostro sguardo «a quel ristretto cerchio in cui ognuno si muove [...]. Quando la tendenza naturale delle sue passioni lo conduce a rendersi premuroso e utile nella sua cerchia, approviamo il suo carattere e amiamo la sua persona grazie a una simpatia con i sentimenti di coloro che hanno con lui rapporti più stretti» ${ }^{54}$.

Per quanto riguarda, invece, le virtù artificiali: esse sono la giustizia (cioè, il rispetto della proprietà), il mantenimento delle promesse e il lealismo ${ }^{55}$. Non esiste un'inclinazione naturale al rispetto della proprietà perché la stessa proprietà è un'invenzione. Sono le condizioni storiche caratterizzate dalla scarsità dei beni e dai limiti della simpatia e della benevolenza che insegnano agli esseri umani ad apprezzare il rispetto del "mio" e del "tuo", in quanto comportamenti reciprocamente vantaggiosi ${ }^{56}$ : «Osservo - questa è la spiegazione di Hume - che è nel mio interesse lasciare a un altro il possesso dei suoi beni, purché egli agisca allo stesso modo nei miei confronti $»^{57}$. Una volta, poi, che questa convenzione si è consolidata, sorgono immediatamente le idee di giustizia e ingiustizia, come quelle di proprietà, diritto e obbligo ${ }^{58}$. In seguito, comunque, con lo sviluppo della società ed i processi di civilizzazione, l'interesse egoistico non viene più riconosciuto ed è perché partecipiamo del dolore altrui che consideriamo vizio qualsiasi infrazione della giustizia. A questo punto, cioè, gli individui non comprendono più che da ogni infrazione della giustizia seguirà disordine, ma non mancano mai di osservare il danno che ricevono, direttamente o indirettamente, per simpatia, dalle ingiustizie altrui. Così mentre

53. Cfr. ivi, p. 642.

54. Ivi, p. 636.

55. Cfr. A.C. Baier, The Cautious Jealous Virtue. Hume on Justice, Harvard University Press, Cambridge (Massachusetts) 2010.

56. Cfr. D. Hume, Trattato sulla natura umana, cit., p. 523.

57. Ivi, p. 517.

58. Cfr. $i v i$, p. 523. 
Metamorfosi dei

Lumi 10.

L'emergenza del sentimento

l'interesse egoistico - afferma Hume - rappresenta il motivo originario che spiega la nascita della giustizia, invece «una simpatia con l'interesse pubblico costituisce la fonte dell'approvazione morale che accompagna questa virtù» ${ }^{59}$. Anche quando è lontana, l'ingiustizia ci risulta sgradevole, perché la consideriamo pregiudizievole per «chiunque si avvicini alla persona che ne è colpevole ${ }^{60}$. Su questi sentimenti, poi, può agire l'artificio dei politici, ma, a differenza di Mandeville, Hume è convinto che se essi non fossero naturali, i politici non potrebbero crearli da zero, nemmeno sfruttando il nostro interesse alla reputazione.

Anche il mantenere le promesse non è una virtù naturale, in quanto la risoluzione di compiere un'azione, da sola, non ci impone mai alcun obbligo ${ }^{61}$ : le promesse sono invenzioni umane, basate sui bisogni e sugli interessi della società ${ }^{62}$, in quanto l'esperienza ci insegna che è vantaggioso se istituiamo simboli o segni «con cui possiamo reciprocamente garantire la nostra condotta in tutti i casi particolari» ${ }^{63}$. Lo stesso vale per il lealismo, che è una virtù perché promuove gli interessi della società, favorendo l'applicazione delle leggi e la risoluzione delle controversie. Tuttavia, secondo Hume, il governo non è sempre necessario: comunque, se il mantenimento delle promesse è vantaggioso, altrettanto lo è l'obbedienza al governo e pertanto soltanto in casi estremi è giusto ribellarci al sovrano ${ }^{64}$.

59. Ivi, p. 528.

60. Cfr. $i v i$, p. 527.

61. Cfr. ivi, p. 547.

62. Cfr. $i v i$, p. 550 .

63. Ivi, p. 553.

64. Cfr. $i v i$, p. 577. 
Metamorfosi dei

Lumi 10.

L'emergenza del

sentimento
Da Jean-Jacques a Julien: Stendhal, Rousseau e la metamorfosi della sensibilità Marco Menin

È per colpa di due o tre princìpi del beylisme che Jean-Jacques Rousseau è stato così infelice.

Friedrich Wilhelm Nietzsche

1. Introduzione: «Tout commence avec Rousseau»

Nel suo studio Nature et Société chez Stendhal: la révolte romantique (1985), l'eminente stendhalien Michel Crouzet riassume con efficacia l'influenza determinante che il pensiero di JeanJacques Rousseau esercitò su Henry Beyle: «Tutto comincia con Rousseau o con il rousseauisme [...]. È di Rousseau in Stendhal che bisogna parlare: come se questi due universi così singolari si intersecassero e, a volte, si confondessero. Rousseau è il "tufo" dell'universo beylista» ${ }^{1}$. Questo rapporto di "filiazione" - come ampiamente mostrato dagli studi dello stesso Crouzet, di Victor Del Litto e, soprattutto, di Raymond Trousson $^{2}$ - ha presto assunto i connotati del parricidio intellettuale: Rousseau, da un lato, è il "maestro" e il modello incontrastato di Stendhal; dall'altro lato, il suo obiettivo polemico spesso tacito, una sorta di "padre-padrone" da cui affrancarsi.

1. M. Crouzet, Nature et Société chez Stendhal: la révolte romantique, Presses Universitaires de Lille, Villeneuve d'Ascq 1985, p. 21.

2. Cfr. M. Crouzet, Nature et Société chez Stendhal, cit.; V. Del Litto, La Vie intellectuelle de Stendhal: genèse et évolution de ses idées, 1802-1821 (1962), Slatkine Reprints, Genève 1997; R. Trousson, Stendhal et Rousseau: continuité et ruptures (1986), Slatkine Reprints, Genève 1999. 
Metamorfosi dei

Lumi 10.

L'emergenza

del

sentimento

Questa presa di posizione non è in sé sorprendente, poiché rispecchia il generale atteggiamento di intere generazioni nei confronti del sentimentalismo rousseauiano. L'ascendente della figura di Rousseau sul mondo letterario della prima metà del diciannovesimo secolo ha una portata sorprendente: da Senancour a Chateaubriand, da Balzac a Hugo, sono innumerevoli gli scrittori la cui formazione giovanile è stata plasmata dalla lettura del filosofo ginevrino e la cui personalità intellettuale si è definita, in seguito, nel confronto critico, sovente aspro, con il medesimo autore.

In Stendhal, tuttavia, l'atteggiamento bifronte nei confronti dell'eredità rousseauiana assume una chiara valenza filosofica, oltre che estetica e letteraria, che non ha sempre ricevuto da parte della critica l'attenzione che merita. Mentre abbondano i contributi che analizzano le influenze dello stile di Rousseau su quello di Stendhal o che indagano minuziosamente le relazioni tra l'autobiografismo del primo e l'égotisme del secondo, molto più radi sono i tentativi di approfondire la ripresa di specifici concetti filosofici rousseauiani nella produzione beylista. In tale prospettiva, gli unici due aspetti che hanno suscitato un certo interesse sono la concezione della memoria e dell'immaginazione, indagate per lo più a partire dalla dottrina della cristallisation teorizzata nel De l'Amour'.

Mi propongo qui di mostrare, attraverso l'analisi di un'opera specifica, Le Rouge et le Noir, come la peculiare etica (ed estetica) del controllo emotivo di Stendhal - che trova la sua trasposizione letteraria privilegiata nel personaggio di Julien Sorel - rappresenti una reazione, filosoficamente consapevole e lucida, per quanto sotto certi aspetti fallimentare, alla dottrina rousseauiana della sensibilité.

3. Su questo aspetto, rinviamo a M.-R. Corredor, voce «Rousseau», in Y. Ansel, P. Berthier e M. Nerlich (a cura di), Dictionnaire de Stendhal, Champion, Paris 2003, pp. 637-639. Cfr. inoltre V. Brombert, Stendhal, lecteur de Rousseau, «Revue des Sciences humaines», 92 (1958), pp. 463-482; G. May, Préromantisme rousseauiste et égotisme stendhalien: convergence et divergence, «L'Esprit créateur», 6 (1966), n. 2, pp. 97-107; Id., Aspects de la sensibilité stendhalienne, «Symposium», 23 (1969), n. 3-4, pp. 303-318; R.N. Coe, Stendhal, Rousseau and the Search of Self, «Australian Journal of French Studies», 16 (1979), pp. 27-47; B. Didier, Stendhal autobiographe, Puf, Paris 1983, in particolare pp. 5-50; G. May, The Rousseauistic Self and Stendhal's Autobiographical Dilemma, «CEuvres et Critiques», 10 (1985), n.1, pp. 19-27; R. Trousson, Rousseau, Stendhal et l'autobiographie, «Bon-À-Tirer, revue littéraire en ligne», 73 (15 novembre 2007); F. Spandri, J.-J. Rousseau, un contre-modèle pour Stendhal?, «Revue d'histoire littéraire de la France», 115 (2015), n. 3, pp. 543-558. 
Da Jean-Jacques a Julien:

Stendhal, Rousseau e la metamorfosi della sensibilità

\section{2. «Rousseau dans Stendhal»}

Uno dei contributi più originali apportati da Rousseau al dibattito filosofico settecentesco riguarda indubbiamente la dottrina della sensibilità. La sensibilité, come ricorda la definizione datane da Jaucourt nell'Encyclopédie, è quella «disposition tendre et délicate de l'âme, qui la rend facile à être émue, à être touchée» ${ }^{4}$. Tale facoltà diventa, a partire dalla seconda metà del Settecento e sino al tournant des Lumières, lo sfondo concettuale condiviso nella spiegazione della sfera emotiva e passionale, inevitabilmente sospesa tra la dimensione fisiologica e quella spirituale, tra il physique e il morat.

Nonostante questa "duplicità" della facoltà sensitiva fosse implicitamente riconosciuta da molti autori coevi a Rousseau (la stessa Encyclopédie presenta due entrées consacrate alla sensibilità ${ }^{6}$ ), egli è il primo a intraprendere - nei Dialogues de Rousseau juge de Jean-Jacques - un'analisi sistematica della "scomposizione" della sensibilità umana in due livelli distinti, ma interdipendenti. A un primo livello, che accomuna l'uomo agli animali, esiste infatti «une sensibilité physique et organique, qui, purement passive, parait n'avoir pour fin que la conservation de notre corps [...] par les directions du plaisir et de la douleur ${ }^{7}$. In tale situazione, che agli occhi di Rousseau è evidentemente pre-morale (e dunque pre-passionale e pre-sociale), si può parlare solo impropria-

4. Enc. XV, Sensibilité, p. 52.

5. Sulla dottrina settecentesca della sensibilità, rinviamo in particolar modo agli studi di F. Baasner, Der Begriff «sensibilité» im 18. Jahrhundert. Aufstieg und Niedergang eines Ideals, Carl Winter, Heidelberg 1988; Id., The Changing Meaning of «Sensibilité»: 1654 till 1704, «Studies in Eighteenth-Century Culture», 15 (1986), n. 1, pp. 77-96. Per indicazioni sulla letteratura più recente cfr. G. Sauder, voce «Sensibilité», in Dictionnaire européen des Lumières, a cura di M. Delon, Puf, Paris 1997, pp. 985-990; F. Piva (a cura di), La sensibilité dans la littérature française au XVII' siècle, Schena-Didier, Fasano-Paris 1998; J. Riskin, Science in the Age of Sensibility: The Sentimental Empiricists of the French Enlightenment, University of Chicago Press, Chicago 2002; P. Goring, The Rhetoric of Sensibility in Eighteenth-Century Culture, Cambridge University Press, Cambridge 2005; S. Gaukroger, The Collapse of Mechanism and the Rise of Sensibility: Science and the Shaping of Modernity, 1680-1760, Oxford University Press, Oxford 2011.

6. L'ambiguità di fondo della sensibilità, considerata di volta in volta una caratteristica fisiologica o una qualità morale, si ritrova appieno nell'Encyclopédie, dove il problema è affrontato in due voci omonime, inerenti rispettivamente l'ambito morale e quello medico. La seconda voce è redatta da Henri Fouquet - esponente di spicco della scuola di Montpellier: cfr. Enc. XV, Sensibilité, sentiment, pp. 38-52.

7. J.-J. Rousseau, Dialogues, in Euvres complètes, a cura di B. Gagnebin e M. Raymond, Gallimard, Paris 1959-1995, 5 voll., vol. I, p. 805. 
Metamorfosi dei

Lumi 10.

L'emergenza

del

sentimento mente di emozione, indicando con tale termine la tonalità piacevole o dolorosa di una determinata azione in relazione ai due principi fondamentali della legge naturale, cioè l'autoconservazione e la pietà. Questi due elementi «istintuali» garantiscono l'equilibrio tra i naturali moti centripeti, che tendono a riferire tutto al soggetto e alla sua esistenza, e gli altrettanto naturali moti espansivi, che lo inducono al contrario ad aprirsi all'alterità.

Accanto (e oltre) a questo livello, esiste tuttavia un'altra sensibilità «active et morale qui n'est autre chose que la faculté d'attacher nos affections à des êtres qui nous sont étrangers». Questo "genere" di sensibilità, irriducibile alla semplice conformazione organica o al meccanicismo corporeo, è dominato da leggi analoghe a quelle magnetiche:

Celle-ci [la sensibilité active et morale], dont l'étude des paires de nerfs ne donne pas la connaissance, semble offrir dans les âmes une analogie assez claire avec la faculté attractive des corps. Sa force est en raison des rapports que nous sentons entre nous et les autres êtres, et, selon la nature de ces rapports elle agit tantôt positivement par attraction, tantôt négativement par répulsion, comme un aimant par ses pôles ${ }^{8}$.

È a questo livello, che coincide con il sentimento e non con la semplice sensazione, che bisogna ricercare il valore normativo dell'emozione:

L'action positive ou attirante est l'œuvre simple de la nature qui cherche à étendre et renforcer le sentiment de notre être; la négative ou repoussante qui comprime et rétrécit celui d'autrui est une combinaison que la réflexion produit. De la première naissent toutes les passions aimantes et douces, de la seconde toutes les passions haineuses et cruelles ${ }^{9}$.

Proprio l'individuazione della categoria del sentimento come principio autonomo delle emozioni consente a Rousseau di affrancarle dalla dimensione della mera necessità fisica, sino ad attribuire loro un ruolo determinante nella condotta morale dell'uomo. È infatti evidente che la bontà o la malvagità di qualsiasi affezione non dipende dalla sua natura, ma dal

8. Ibid.

9. Ibid. 
Da Jean-Jacques a Julien:

Stendhal, Rousseau e la metamorfosi della sensibilità suo uso, come conferma anche la testimonianza autobiografica: «L'épée use le fourreau, dit-on quelquefois. Voilà mon histoire. Mes passions m'ont fait vivre, et mes passions m'ont tué» ${ }^{10}$.

Per spiegare la dinamica che indirizza le passioni verso il bene o verso il male, che è di per sé irriducibile allo studio del funzionamento di ogni singola affezione, viene introdotta, sempre nei Dialogues, un'ulteriore distinzione all'interno della sensibilità attiva e morale (o sentimento). Accanto a una «sensibilité positive [qui] dérive immédiatement de l'amour de soi» ed «est une pure affaire de sentiment ou la réflexion n'entre pour rien», esiste una «sensibilité négative», diretta espressione di quella degenerazione dell' amour de soi che è l'amour propre: "Aussitôt qu'on prend l'habitude de se mesurer avec d'autres, et de se transporter hors de soi pour s'assigner la première et meilleure place, il est impossible de ne pas prendre en aversion tout ce qui nous surpasse, tout ce qui nous rabaisse, tout ce qui nous comprime, tout ce qui étant quelque chose nous empêche d'être tout» ${ }^{11}$.

La dottrina rousseauiana della sensibilité, che qui abbiamo ricostruito per sommi capi ${ }^{12}$, sarà destinata non solo a fornire a Stendhal il materiale - il "tufo" di cui parla Crouzet - con cui egli costruisce la propria "filosofia" dell'emozione, ma anche a diventarne il banco di prova, a causa principalmente di quattro assunzioni che essa implica. La prima e più evidente conseguenza della dottrina rousseauiana è l'esistenza di un rapporto diretto e bidirezionale tra la dimensione fisico-

10. J-J. Rousseau, Confessions, $O C$ I, p. 219. Come studi generali sull'emozione nel pensiero di Rousseau, cfr. L. Mall e B. Weltman-Aron (a cura di), De l'émotion chez Rousseau, «L'Esprit Créateur», 52 (2012), n. 4; J. Berchtold (a cura di), Rousseau, passionnément: «Mes passions m'ont fait viure, et mes passions m'ont tué», Éditions Mare et Martin, Paris 2013.

11. J.-J. Rousseau, Dialogues, $O C \mathrm{I}$, p. 806. Mentre l'amore di sé (amour de soi) è un sentimento assoluto, naturale e buono per definizione perché assicura l'autoconservazione dell'individuo ed esprime il suo diritto alla vita, l'amor proprio (amour propre) è invece sempre negativo, perché, nascendo dal confronto con gli altri, si configura come sentimento sociale ed è quindi subordinato all'opinione. Cfr. Y. Sakakura, Essai sur «l'amour-propre» chez J.-J. Rousseau, «Études J.-J. Rousseau», 7 (1995), n. 1, pp. 223-239.

12. Per un'analisi più approfondita della questione mi permetto di rinviare a M. Menin, Il libro mai scritto. La morale sensitiva di Rousseau, Il Mulino, Bologna 2013, pp. 129-154; ed. francese La morale sensitive de Rousseau. Le livre jamais écrit, L'Harmattan, Paris 2019, pp. 127-150. 
Metamorfosi dei

Lumi 10.

L'emergenza

del

sentimento fisiologica e quella morale, che trova nell'emozione la sua manifestazione privilegiata.

In secondo luogo, il fatto di far derivare l'emozione (intesa come espressione della sensibilità morale positiva) da un principio antropologicamente buono come l'amour de soi consente a Rousseau d'instaurare un rapporto lineare, basato su una proporzionalità diretta, tra moralità ed effusione sentimentale. Oltre che in tutta la produzione romanzesca, questo aspetto costituisce un leitmotiv della rappresentazione autobiografica, come conferma (tra i numerosi esempi che si potrebbero scegliere) il profilo di "Jean-Jacques" delineato da "Rousseau" nei Dialogues:

Les affections auxquelles il a le plus de pente se distinguent même par des signes physiques. Pour peu qu'il soit ému ses yeux se mouillent à l'instant. Cependant jamais la seule douleur ne lui fit verser une larme; mais tout sentiment tendre et doux, ou grand et noble dont la vérité passe à son cœur lui en arrache infailliblement. Il ne saurait pleurer que d'attendrissement ou d'admiration: la tendresse et la générosité sont les deux seules cordes sensibles par lesquelles on peut vraiment l'affecter. Il peut voir ses malheurs d'un oil sec, mais il pleure en pensant à son innocence, et au prix qu'avait mérité son cœur ${ }^{13}$.

Questo ritratto, tracciato con pennellate patetiche, mette bene in risalto un'ulteriore caratteristica dell'emozione secondo Rousseau. Essa non affonda solo le proprie radici nell'amor di sé, ma anche nella pietà, ossia - per riprendere la celebre definizione contenuta nel secondo Discours - la «virtue naturelle $[. .$.$] qui nous inspire une répugnance naturelle$ à voir périr ou souffrir tout être sensible et principalement nos semblables» ${ }^{14}$. Essa è dunque un sentimento simpatetico, che instaura una relazione trasparente e immediata tra l'interiorità e l'esteriorità, svelando la verità della prima attraverso la seconda: "[Les émotions] des cœurs ardents et sensibles étant l'ouvrage de la nature, se montrent en dépit de celui qui les a; leur première explosion purement machinale est indépendante de sa volonté» ${ }^{15}$.

13. J.-J. Rousseau, Dialogues, OC I, p. 825.

14. J.-J. Rousseau, Discours sur l'inégalité, OC III, p. 126.

15. J.-J. Rousseau, Dialogues, OC I, p. 8632. 
Da Jean-Jacques a Julien:

Stendhal, Rousseau e la metamorfosi della sensibilità
In questo nesso originario tra pietà e passione risiede il terzo aspetto determinante della dottrina della sensibilità di Rousseau, ossia il ruolo direttivo che egli assegna al sentimento e all'emozione a discapito della ragione. Grazie alla sua capacità di essere autentica, cioè involontaria e spontanea, l'emozione rappresenta infatti non solo uno scarto determinante tra l'uomo di natura (amorale e insensibile) e l'homme de l'homme (morale ed emotivo), ma può essere considerata una vera e propria fonte di normatività. Proprio in quanto si sottrae al controllo del soggetto e non può essere manipolata, essa è capace di mettere in contatto l'individuo con il suo prossimo, sino a condurre - come viene sottolineato non a caso nella Préface dialogata della Nouvelle Héloïse - a una perfetta condivisione dell'interiorità: «Cependant on se sent l'âme attendrie; on se sent ému sans savoir pourquoi. Si la force du sentiment ne nous frappe pas, sa vérité nous touche, et c'est ainsi que le cœur sait parler au cour» ${ }^{16}$.

L'ultima, e per certi aspetti più importante conseguenza della peculiare visione rousseauiana della sensibilità, risiede nella constatazione della natura intrinsecamente filosofica della narrazione letteraria (sia romanzesca, sia autobiografica). Mentre una riflessione astratta sulla dimensione emotiva (basti pensare ai traités des passions tipici del Seicento) rimane sterile, in quanto non è in grado di colpire la sensibilità morale del lettore, la dinamica finzionale del romanzo $f a$ agire l'emozione, sino a tradurre in pratica la dottrina della pietà, incentrata sull'immedesimazione immaginativa del lettore nel personaggio. Questo meccanismo, al centro della già menzionata Préface dialogata della Nouvelle Héloïse, verrà immortalato con finezza da Diderot nell'Éloge de Richardson, altro manifesto del valore intrinsecamente filosofico della letteratura sentimentale: "Celui qui agit, on le voit, on se met à sa place ou à ses côtés, on se passionne pour ou contre lui; on s'unit à son rôle, s'il est vertueux; on s'en écarte avec indignation, s'il est injuste et vicieux» ${ }^{17}$. Grazie alla mediazione della

16. J.-J. Rousseau, Nouvelle Héloïse, Préface, $O C$ II, p. 15. Sull'immagine della comunione tra i cuori, mi permetto di rinviare a M. Menin, When One Heart Can Speak to Another: The Role of Tenderness in Rousseau's Theory of Passions, «Intellectual History Review», 28 (2018), n. 3, pp. 349-368.

17. D. Diderot, Éloge de Richardson, in Euvres complètes, a cura di H. Dieckmann, J. Fabre (poi J. Varloot) e J. Proust, Hermann, Paris 1975-2004, 33 voll., vol. XIII, pp. 192-193. 
Metamorfosi dei

Lumi 10.

L'emergenza

del

sentimento sensibilità morale, che al contempo agisce nella narrazione e consente al lettore di reagire appropriatamente, il testo diventa un vero e proprio spazio sociale, che permette d'instaurare un'efficace comunicazione morale tra gli individui.

L'inscindibilità del pensiero beyliano da quello rousseauiano, e al contempo l'ambiguità che connota questo rapporto, è a nostro modo di vedere giustificabile proprio alla luce della dottrina della sensibilità. Sthendhal prova infatti a confutare alcuni degli assunti di Rousseau su questo aspetto (nello specifico la moralità dell'effusione sentimentale e il ruolo direttivo del sentimento) rimanendo tuttavia al contempo sinceramente convinto della bontà di altri assunti (il rapporto reciproco tra fisico e morale e, soprattutto, l'idea che la sensibilità possa essere indagata solo attraverso la dimensione narrativa). L'esito di questo détourè una riflessione profonda e dolorosa, scandita da numerose prese di posizione e successivi rinnegamenti.

3. "Dérousseauiser mon jugement»: la tela di Penelope di Stendhal Tutta la produzione di Henry Beyle - dagli scritti romanzeschi a quelli autobiografici, sino a giungere a quelli più strettamente teorici - è attraversata da uno sforzo costante, anche se spesso fallimentare, di prendere congedo dal modello rousseauiano, che ha indelebilmente segnato la sua formazione letteraria e filosofica. Iniziato agli scritti di Rousseau dal nonno, il docteur Gagnon (che aveva assistito al soggiorno dello stesso Rousseau a Grenoble, nel 1768), Stendhal scopre sin da ragazzo la Nouvelle Héloïse e l'Émile, letti prima del suo ingresso all'École centrale. Nel 1800, ormai sotto-luogotenente dei dragoni in marcia per raggiungere la sua armata a Milano, egli si reca a visitare la casa natale di Jean-Jacques a Ginevra e sogna i personaggi della Julie passando per Vevey e Meillerie, mentre l'amata Italia gli fa tornare alla mente alcuni dei più celebri episodi delle Confessions.

Tra il 1802 e il 1814 Stendhal intraprende un difficile processo di "emancipazione" da Rousseau, che continua ciononostante a leggere assiduamente e a citare diffusamente nel Journal e nella corrispondenza. Il 21 novembre del 1804, in seguito a un periodo di studio e riflessione, arriva a formulare il celebre proposito di «dérousseauiser mon jugement en lisant Destutt [de Tracy], Tacite, Prévost de Genève, 
Da Jean-Jacques a Julien:

Stendhal, Rousseau e la metamorfosi della sensibilità
Lancelin» ${ }^{18}$. Si tratta in questo passo chiaramente di contrastare e neutralizzare l'eccessiva influenza della sensibilità sulla razionalità, di far prevalere il conoscere sul sentire, ponendo l'individuo al riparo dai patologici eccessi di una sensibilità ormai mutatasi in sensiblerie ${ }^{19}$. Rousseau, in questa prospettiva, diviene l'emblema della posizione filosofica che attribuisce un ruolo morale direttivo al cuore e all'emozione. Non a caso, nella sua lotta a favore della razionale maîtrise de soi contro la spontanea effusione sentimentale, Beyle sceglie come alleati gli Idéologues, che cita apertamente nella pagina del Journal come antidoto al rousseauisme, e Helvétius, l'âme froide che riconduceva la dimensione passionale alla sensazione trasformata.

Il beylisme si costituisce così lentamente contro Rousseau, come testimonia il paradossale (e incompiuto) progetto di fornire, nella Vie de Henry Brulard, una «traduction des Confessions de Jean-Jacques en style à moi» ${ }^{20}$. Ricostruendo la propria esistenza, lo stesso Stendhal ammette di essersi considerato, perlomeno sino al 1800, una «âme sensible», incline al pianto e alla commozione:

Je trouvai les comédies de Destouches et l'une des plus ridicules m'attendrit jusqu'aux larmes;

La lecture de La Nouvelle Héloïse et les scrupules de $\mathrm{S}$ [aint]-Preux me formèrent profondément honnête homme; je pouvais encore, après cette lecture faite avec larmes et dans des transports d'amour pour la vertu, faire des coquineries, mais je me serais senti coquin. Ainsi c'est un livre lu en grande cachette et malgré mes parents qui m'a fait honnête homme ${ }^{21}$.

Il grande trauma dell'infanzia di Henry è legato proprio alla concezione sentimentalista della sensibilità (o, per meglio

18. Stendhal, Journal, 21 novembre 1804, in Euvres intimes, a cura di V. Del Litto, Gallimard, Paris 1981-1982, 2 voll., vol. I, p. 152. Pierre Prévost e Pierre-François Lancelin sono due intellettuali riconducibili al milieu degli Idéologues.

19. Il neologismo «sensiblerie» fu coniato da Mercier, per indicare un patologico e inautentico eccesso di sensibilità: «Sensiblerie. Fausse sensibilité; elle n'est que trop à la mode. Les êtres privés de la vraie sensibilité, abondent en Sensiblerie» (L.-S. Mercier, Néologie: ou vocabulaire de mots nouveaux, à renouveler, ou pris dans des acceptions nouvelles, Moussard-Maradan, Paris 1801, 2 voll., vol. II, p. 246).

20. Stendhal, Journal littéraire, a cura di V. Del Litto, Cercle du Bibliophile, Genève 1970, 3 voll., vol. III, p. 33.

21. Stendhal, Vie de Henry Brulard, in Euvres intimes, cit., vol. II, pp. 619 e 716-717. 
Metamorfosi dei

Lumi 10.

L'emergenza

del

sentimento

dire, alla sua volgarizzazione popolare) e all'identificazione tra lacrime e virtù ${ }^{22}$. Il bambino è infatti ingiustamente accusato di essere «insensibile» perché non ha pianto al funerale dell'adorata madre: «Ma tante Séraphie osa me reprocher de ne pas pleurer assez. Qu'on juge de ma douleur et de ce que je sentis! Mais il me semblait que je la reverrais le lendemain, je ne comprenais pas la mort ${ }^{23}$.

Se dunque, sino ai vent'anni circa, il grande obiettivo di Stendhal è quello di uniformarsi ai presupposti morali e stilistici del sentimentalismo, negli anni successivi si afferma in lui con sempre più forza la volontà di controllare una sensibilità avvertita come iper-acuta ed eccessiva: «Il sentimentalismo rousseauiano, alla luce della sua esperienza personale e dell'insegnamento dei philosophes, gli pare una malattia da cui è urgente guarire ${ }^{24}$. Lo strumento più efficace per portare a termine questa impresa terapeutica - sia a livello filosofico sia a livello letterario - è lo sviluppo di una razionale «distanza» rispetto alla sfera dell'emotività. Non solo la verità pare a Stendhal inconciliabile con il lirismo sentimentalista, che «traveste» e deforma la realtà del vissuto, ma l'ideale del moi embelli, tipico del rousseauismo, finisce con il precludere al soggetto la felicità ${ }^{25}$.

Tale progetto si risolve tuttavia in una tensione mai risolta, paragonabile - per riprendere un'efficace metafora di Raymond Trousson - alla tela di Penelope: «I suoi sforzi di dérousseauisation, per una decina d'anni, ricordano sovente il lavoro di Penelope: un'impresa senza fine, in cui ogni mese disfa il lavoro fatto precedentemente ${ }^{26}$. Come uomo e come artista, Stendhal vive il paradosso di non poter né seguire né rinnegare del tutto il proprio maestro. Lo stile e i temi cari al sentimentalismo rousseauiano - spesso declinati a con-

22. Sulla complessa ricostruzione del modello del sentimentalismo, cfr. R.F. Brissenden, Virtue in Distress: Studies in the Novel of Sentiment from Richardson to Sade, Macmillan, London 1974, pp. 11-64 («Sentimentalism»: An Attempt at Definition); D. Denby, Sentimental Narrative and the Social Order in France, 1760-1820, Cambridge University Press, Cambridge 1994, pp. 71-94 (Towards a Model of the Sentimental Text) e M. Cohen, The Sentimental Education of the Novel, Princeton University Press, Princeton 1999.

23. Stendhal, Vie de Henry Brulard, cit., p. 556.

24. R. Trousson, Stendhal et Rousseau, cit., p. 72.

25. Cfr. R. Bauer, Julie et Julien ou le problème du bonheur chez J.J. Rousseau et Stendhal, «Romanische Forschungen», 65 (1954), n. 3-4, pp. 378-391.

26. R. Trousson, Stendhal et Rousseau, cit., p. 39. 
Da Jean-Jacques a Julien:

Stendhal, Rousseau e la metamorfosi della sensibilità trario - ossessionano letteralmente la sua produzione, dal De l'Amour all'Armance, sino alla Chartreuse de Parme, l'opera più rousseauiana nei toni.

\section{4. «Une sensibilité sotte»: le lacrime di Julien}

È tuttavia in Le Rouge et le Noir, del 1830, che Stendhal si propone di dissipare una volta per tutte le illusioni sentimentali della giovinezza e di spogliare la creazione romanzesca da qualsiasi traccia di affettazione e di idealismo. Stendhal mette qui apertamente in scena lo scontro tra la sensibilité, ormai irrimediabilmente trasformatasi in sensiblerie, e una nuova etica (ed estetica) del controllo emotivo. La contrapposizione a Rousseau, alla cui Julie sono consapevolmente improntati alcuni dei personaggi principali del romanzo (su tutti, Madame de Rênal), si articola così su un duplice livello. Per quel che concerne lo stile, Stendhal adotta un periodare sincopato e duro, adatto a contrastare le lunghe descrizioni enfatiche e i tableux edificanti tipici della letteratura partecipativa. Questa riforma formale va di pari passo con una rielaborazione teorica del paradigma rousseauiano concernente il valore morale attribuito all'emozione, che trova espressione nella complessa figura del protagonista del romanzo. Oltre a essere il simbolo della crisi politica e sociale vissuta dalla Francia nei primi decenni del diciannovesimo secolo, Julien Sorel è l'emblema di una generazione ormai consapevole dell'insufficienza della sensibilità, ma che ciononostante non riesce a prendere congedo da essa.

Ciò che distingue il protagonista di Stendhal da molti altri personaggi romanzeschi che si propongono di contrastare il modello antropologico imposto da Rousseau (basti pensare ai libertini di Sade e Laclos) è la sua intima adesione alla dottrina rousseauiana della sensibilità. La drammaticità (e il fascino) della figura di Julien scaturisce infatti dalla sua natura intrinsecamente e irrimediabilmente sentimentale, che egli proverà per tutto il tempo a rinnegare. Questa battaglia interiore trova espressione in particolar modo nelle lacrime - emozione privilegiata del sentimentalismo in generale e dell'autobiografismo rousseauiano nello specifico ${ }^{27}$ - la cui

27. Come studio generale sull'argomento, mi permetto di rinviare a M. Menin, La filosofia delle lacrime. Il pianto nella cultura francese da Cartesio a Sade, Il Mulino, Bologna 2019. 
Metamorfosi dei

Lumi 10.

L'emergenza

del

sentimento

alterna effusione e repressione rappresenta parte integrante della "metamorfosi", al tempo stesso sociale e antropologica, del personaggio ${ }^{28}$.

Come Stendhal, il giovane Sorel plasma il proprio ideale di vita a partire dalla lettura delle Confessions di Rousseau, «le seul livre à l'aide duquel son imagination se figurait le monde ${ }^{29}$. Questa sensibilità di stampo rousseauiano si coniuga tuttavia in lui con una disperata sete di ascesa sociale, che lo induce ad adottare - in completa dissonanza con l'esaltazione dell' être a discapito del paraître tipica del Ginevrino - un atteggiamento amorale, se non apertamente immorale.

L'intera narrazione romanzesca si può allora interpretare come lo scontro tra il codice settecentesco della sensibilità, incentrato sulla spontaneità dell'emozione, e un'etica dell'onore, tipicamente ottocentesca, che individua al contrario nel controllo razionale dell'emozione la condizione di possibilità stessa di un'“autentica" sensibilità, non eccessiva e non patologica $^{30}$. La tensione concettuale che scaturisce da un simile scontro è, nella prospettiva della filosofia dell'emozione, tanto evidente quanto feconda: Stendhal rivendica un ruolo morale fondativo ("a monte") della dimensione affettiva, incentrato sulla sua spontaneità e sulla sua autonomia dalle altre facoltà umane, suggerendo però al contempo la necessità di una sua valutazione "a valle", ossia in base al controllo razionale e volontario che il soggetto può esercitare su di essa.

Per questo motivo, lungi dal ricercare l'immedesimazione empatica con il prossimo, Julien ambisce alla rappresentazione esterna dei sentimenti finalizzata alla manipolazione dell'emozione altrui. Da queste paradossali premesse discen-

28. L'unica monografia sul soggetto è, a mia conoscenza, G. Pascal, Rires, sourires et larmes chez Stendhal: une initiation poétique, Droz, Genève 1993. Questo studio ci pare tuttavia viziato da un eccessivo uso di categorie psicanalitiche e da una scarsa attenzione alla ricostruzione storiografica della teoria stendhaliana delle passioni. Più attenti a questo aspetto sono i contributi di L. Cellier, Rires, sourires et larmes, dans «La Chartreuse de Parme», in Omaggio a Stendhal, Aurea Parma, Parma 1967, pp. 18-33 e Id., Rires, sourires et larmes dans "Le Rouge et le Noir», in De J. Lemaire de Belges à J. Giraudoux, Nizet, Paris 1970, pp. 277-297.

29. Stendhal, Le Rouge et le Noir, in Romans et nouvelles, a cura di H. Martineau, Gallimard, Paris 1952, 2 voll., vol. I, p. 235.

30. Questa tensione era già ben presente, a livello teorico, nel De l'Amour, come evidenziato da M. Gill, Self-Control and Uncontrollable Passion in Stendhal's Theory of Love, «French Studies», 69 (2015), n. 4, pp. 462-478. 
Da Jean-Jacques a Julien:

Stendhal, Rousseau e la metamorfosi della sensibilità de, da un lato, la sua naturale inclinazione al pianto (inteso, in termini rousseauiani, come prova di sensibilità) e, dall'altro lato, la profonda vergogna (del tutto anti rousseauiana) che il ragazzo nutre nei confronti di ogni sua spontanea manifestazione emotiva, vista come un ostacolo alla realizzazione di quell'arte della finzione che dovrebbe consentirgli di elevarsi alla condizione nobiliare. Non è allora un caso che, all'inizio del romanzo, il fatale incontro tra Julien e Madame de Rênal sia caratterizzato proprio dall'effusione mal dissimulata del pianto di lui:

Je viens pour être précepteur, madame, lui dit-il enfin, tout honteux de ses larmes qu'il essuyait de son mieux [...]. Madame de Rênal regardait les grosses larmes, qui s'étaient arrêtées sur les joues si pâles d'abord et maintenant si roses de ce jeune paysan ${ }^{31}$.

La vergogna è destinata a diventare il sentimento predominante di Julien nei confronti della propria emotività, come emerge con nettezza di fronte alla crescente consapevolezza dell'autenticità dell'amore nutrito per Madame de Rênal, che doveva invece limitarsi ad essere un sentimento simulato: «Julien avait honte de son émotion; pour la première fois de sa vie, il se voyait aimé; il pleurait avec délices et alla cacher ses larmes dans les grands bois au-dessus de Verrières» ${ }^{32}$. Lo stesso rifiuto delle lacrime si estende dalla sfera dell'amore a quella delle relazioni sociali in senso lato. Particolarmente significativo è l'episodio della visita resa dal giovane precettore a Monsieur de Valenod, il ricco direttore dell'ospizio per i poveri di Verrières, e alla consorte. Qui Julien rimane dapprima disgustato dall'affettazione di sensibilità con cui viene ricevuto dalla padrona di casa, «qui lui présenta ses enfants les larmes aux yeux» ${ }^{33}$, per poi commuoversi invece intimamente pensando a come, proprio nello stesso momento in cui si svolgeva il lussuoso banchetto a cui stava prendendo parte, i detenuti dell'istituto patissero la fame. La loro unica consolazione è una canzone intonata a squarciagola, che viene però crudelmente messa a tacere dal cinico Valenod: 
Metamorfosi dei Lumi 10. L'emergenza del sentimento
J'ai fait imposer silence aux gueux. Ce mot fut trop fort pour Julien; il avait les manières, mais non pas encore le cœur de son état. Malgré toute son hypocrisie si souvent exercée, il sentit une grosse larme couler le long de sa joue [...]. Par bonheur, personne ne remarqua son attendrissement de mauvais ton ${ }^{34}$.

L'identificazione tra sensibilità e debolezza, intesa come una patologica faiblesse, si accentua con il procedere della scalata sociale di Julien. Anche quando, grazie alla sua ipocrisia, riesce a farsi strada nella mondanità parigina, sino a conquistare il cuore di Mathilde, la figlia del marchese de la Mole, il ragazzo non riesce a tenere sotto controllo del tutto quella che definisce con disprezzo una sensibilité sotte, che lo fa commuovere alla semplice vista del canapé prediletto di Mathilde, di cui si è innamorato nuovamente suo malgrado: «À la vue du canapé bleu, il fut ému jusqu'aux larmes; bientôt ses joues devinrent brûlantes. Il faut user cette sensibilité sotte, se dit-il avec colère; elle me trahirait ${ }^{35}$.

Il sistematico sforzo di autocontrollo del giovane sulla propria emotività - «Comment tuer cette sensibilité si humiliante? ${ }^{36}$, si domanda al termine di un altro celebre episodio in cui sfida a duello un passante reo di fissarlo con alterigia - è destinato tuttavia allo scacco. Quando una lettera di delazione di Madame de Rênal manda a monte il matrimonio con Mathilde, Julien, che vede tutti i suoi sogni e le sue speranze distrutte, fa ritorno a Verrières, raggiunge l'ex amante in chiesa e la ferisce con un colpo di pistola. Egli viene così imprigionato e condannato alla ghigliottina nonostante tutti gli intrighi architettati da Mathilde e l'affetto di Madame de Rênal (che è sopravvissuta e che, colta dai rimorsi, lo perdona) in seguito a un processo che si può interpretare come una vera e propria requisitoria contro la visione sentimentalista dell'emozione. Imputato e giudice al contempo, Julien è costantemente ossessionato dallo sguardo altrui, ossia dall'interpretazione che il pubblico darà alle sue manifestazioni emotive ${ }^{37}$.

34. Ivi, p. 348.

35. Ivi, p. 598.

36. Ivi, p. 472.

37. Si tratta di una tematica tipicamente rousseauiana. Cfr. J. Starobinski, L'Eil vivant, Gallimard, Paris 1961; trad. it. di G. Guglielmi, L'occhio vivente: Studi su Corneil- 
Da Jean-Jacques a Julien:

Stendhal, Rousseau e la metamorfosi della sensibilità
Inizialmente Julien valuta con profondo disprezzo l'interesse suscitato dal suo processo, attribuendolo a una sorta di esibizione della sensibilità: «Je ne serai que trop longtemps exposé en spectacle à tous mes ennemis [...]. Il n'en est pas un qui ne désire ma condamnation, sauf à pleurer comme un sot quand on me mènera à la mort ${ }^{38}$. Tuttavia, quando viene a contatto con la folla, «il fut obligé de reconnaître que sa présence inspirait au public une pitié tendre» ${ }^{39}$. Commosso dalle lacrime di coloro che sperano nella sua assoluzione, «peu à peu la sécheresse d'âme de Julien disparut devant les marques d'intérêt dont il était évidemment l'objet» ${ }^{40}$. Persino quando viene condannato a morte, mentre «toutes les femmes fondaient en larmes» e svenivano, il ragazzo non riesce a fare a meno di pensare a come «ce pauvre président des assises, tout juge qu'il est depuis nombre d'années, avait la larme à l'œil en me condamnant ${ }^{41}$. Gli ultimi giorni di vita di Julien sono così caratterizzati da una vera e propria ciclotimia tra una sorta di rivincita della valutazione simpatetica del pianto e una sua sprezzante condanna. Questa dolorosa tensione è sottolineata dal giovane Sorel in una delle ultime conversazioni che ha con Mathilde: «J'ai le cour facile à toucher; la parole la plus commune, si elle est dite avec un accent vrai, peut attendrir ma voix et même faire couler mes larmes. Que de fois les cœurs secs ne m'ont-ils pas méprisé pour ce défaut!» ${ }^{42}$. Una situazione analoga si ripresenta poco dopo quando, dinnanzi ai rimproveri del padre cinico e severo, "Julien ne put retenir ses larmes. Quelle indigne faiblesse! se dit-il avec rage ${ }^{43}$.

La conclusione ambigua e beffarda di Le Rouge et le Noir, che si chiude con la descrizione, ostentatamente sentimentalista, del funerale eterodosso di Julien e della disperazione delle sue due amanti, si può considerare come una delle trasposizioni letterarie più riuscite della profonda metamorfosi

\section{le, Racine, Rousseau, Stendhal, Freud, Einaudi, Torino 1975 e D. Balestra, Lo specchio assente. J.-J. Rousseau e lo sguardo altrui, Cappelli, Bologna 1989.}

38. Stendhal, Le Rouge et le Noir, cit., p. 671.

39. Ivi, p. 672 .

40. Ivi, p. 673 .

41. Ivi, pp. 675 e 676 .

42. Ivi, p. 678 .

43. Ivi, p. 688. 
Metamorfosi dei

Lumi 10.

L'emergenza del

sentimento a cui era andata incontro la dottrina della sensibilità. Ormai chiaramente patologica se abbandonata alla sua spontaneità, tale facoltà richiede con sempre più forza un controllo razionale in grado di ovviare al drammatico destino dell' homme sensible, efficacemente riassunto dalle ultime parole che Julien - forse per la prima e ultima volta davvero sincero - rivolge a Fouqué prima di salire sul patibolo: «Pour de l'émotion, je ne puis en répondre» ${ }^{44}$. 
Metamorfosi dei

Lumi 10.

L'emergenza del

sentimento

\section{L'Encyclopédie Méthodique des passions} au début du XIX ${ }^{\mathrm{e}}$ siècle

Daniel Teysseire

Les passions sont de toutes les époques historiques et donc de l'époque moderne. Même chose, peu ou prou, pour le discours sur les passions ${ }^{1}$. Mais à la fin de l'époque moderne celui-ci va prendre un tournant qui peut s'apparenter à une rupture épistémologique. Discours moral, voire moralisant pour ne pas dire moralisateur - effet de la Réforme et de la Contre-Réforme - il va devenir médical et même tout simplement thérapeutique.

Notre point de départ nous est donné par cet instrument lexico-bibliographique du temps initial de la crise de la conscience européenne: le Dictionnaire universel, Contenant tous les Mots François tant vieux que modernes, E les Termes de toutes les Sciences et des Arts [...], de Furetière. En son tome III de 1690 édité aux Pays-Bas, la quatrième entrée de l'article PASSION, celle de l'acception morale de ce mot se

1. Pour la mise en situation générale, voir la toute récente Histoire des émotions ss. la dir. d'A. Corbin, J.-J. Courtine et G. Vigarello, Seuil, Paris 2016 et 2017, 3 vols. Il n'y est pas beaucoup question de «passions», si ce n'est dans le vol. I De l'Antiquité aux Lumières, dans la partie sur l'Âge moderne, pp. 241-243. La p. 242 indiquant les deux types de passions selon Thomas d'Aquin: les concupiscibles et les irascibles, déjà mentionnées en 1992 par C. Talon-Hugon dans son article de Corpus 20/21 référencé ci-dessous dans la note suivante. 
Metamorfosi dei

Lumi 10.

L'emergenza del sentimento

termine par ces lignes de références: «Voyez l'Abrégé de Gassendi, \& sur tout Monsieur Descartes, qui a fait un beau Traité des Passions d'une manière physique. Coeffeteau a fait le Tableau des Passions; La Chambre, les Caractères des Passions; le Père Senault, l'Usage des Passions». Ainsi, entourant Descartes et son traité des passions de l'âme valorisé, nous avons quatre auteurs d'ouvrages sur lesdites passions datant d'une, voire de deux générations d'avant celle de la crise de la conscience européenne à l'époque de laquelle se situe - comme déjà dit - le Dictionnaire de Furetière. En suivant la chronologie ces cinq ouvrages sur les passions s'ordonnent de la manière suivante:

1620: Tableau des Passions humaines, de leurs causes, et de leurs effets du Père Coeffeteau (1574-1623).

1640-1662: Les Charactères des Passions du Docteur Cureau de la Chambre (1594[?]-1669).

1641: De l'Usage des Passions du Père Senault (1599[?]1672).

1649: Les Passions de l'Âme de Descartes (1596-1650).

1674: Abrégé de la Philosophie de Mr. Gassendi du Docteur Bernier (1620-1688)².

Quelle belle base pour le travail des Lumières que ces cinq ouvrages! Ne leur fournissent-ils pas, en effet, diverses typologies des passions et une double approche de celles-ci: celle de religieux, les Pères Coeffeteau et Senault, chargés ès-qualité de la morale, et celle de médecins, De La Chambre et Bernier, plus portés vers des explications physiologistes des passions. Tous ayant en commun la même finalité, la seule qui vaille dans et pour la société chrétienne: la finalité morale de la maîtrise des passions. Car suivre ou même seulement s'abandonner à des passions, c'est non seulement entrer dans le désordre individuel et social, être un «mauvais sujet», mais aussi et surtout hypothéquer l'avenir dans l'Au-delà par le péché, et donc son Salut. D’ailleurs, en ôtant simplement la dimension chrétienne, l'organe des

2. Dans l'œuvre de Gassendi (1592-1655), «La théorie des passions fait l'objet du livre X de la troisième section de la 'Physique' du Syntagma intitulé 'De Appetitu et Affectibus Animae» (C. Talon-Hugon, «La question des passions, occasion de l'évaluation de l'humanisme de Gassendi", Corpus n. 20/21, $2^{\text {nd }}$ semestre 1992, p. 37), ledit Syntagma philosophiae Epicuri [...] étant de 1649. Comme le traité des Passions de Descartes! 
L'Encyclopédie Méthodique des passions au début du XIX siècle
Lumières éclatantes ou rayonnantes qu'est en quelque sorte l'Encyclopédie reste dans cette perspective de la finalité morale de la maîtrise des passions. En effet, après avoir posé une typologie de celles-ci, la partie «II» de la première entrée de l'article «PASSIONS (Philos. Logique, Morale.)»- sans doute de Jaucourt - conclut en prônant leur modération par la raison ${ }^{3}$.

1. La morale des passions: inventaire des articles PASSION/S $d u$ «Dictionnaire de Logique, Métaphysique et Morale» de l'Encyclopédie Méthodique.

Qu'en est-il, une génération plus tard, pour les Lumières fulminantes, celles de l'Encyclopédie méthodique, avec ses multiples dictionnaires correspondant à autant de champs scientifiques ou disciplinaires? Nous l'allons voir à partir d'un inventaire - ô combien perfectible! - de quelques «Dictionnaires» de la Méthodique:

1788: Dictionnaire de «Logique et Métaphysique», T. II, pp. 92G1 => 105D4: «PASSION, s.f., «Explication particulière de tous les changemens qui arrivent au corps $\mathcal{E} \Xi$ à l'âme dans les passions».

1791: Dictionnaire de «Logique, Métaphysique et Morale», T. IV, pp1G41=>21D144: «PASSIONS, s. f.».

1793/4 (= «An II»): Dictionnaire de «Philosophie ancienne et moderne», T. III: dirigé par Naigeon, il ne contient pas d'article «PASSION/S».

1797: Dictionnaire d' «Art militaire», T. IV de «supplément», pp. 806G20 => D11: «PASSIONS».

1824: Dictionnaire de «Médecine. Contenant: $1^{\circ}$. L'Hygiène. $2^{\circ}$. La Pathologie. $3^{\circ}$. La Séméiotique \& La Nosologie. $4^{\circ}$. La Thérapeutique ou Matière médicale. $5^{\circ}$. La Médecine militaire. $6^{\circ}$. La Médecine vétérinaire. $7^{\circ}$. La Médecine légale. $8^{\circ}$. La Jurisprudence de la Médecine \&

3. Encyclopédie ou Dictionnaire raisonné des Sciences, des Arts et des Métiers, Par une Société de gens de lettres, Samuel Faulche \& Compagnie, Neufchastel [Paris]1765, T. XII, pp. 142 col. a - 146 col. a. Lignes 47-51 de cette dernière: «Triste tableau de l'état où l'homme est réduit par ses passions! Environné d'écueils, poussé par mille vents contraires, pourrait-il arriver au port? Oui, il le peut; il est pour lui une raison qui modère les passions [...]».

4. Le premier chiffre indique la page du volume, le deuxième la colonne de la page, le troisième la ligne. Entre 1788 et 1791 ce dictionnaire de «Logique et Métaphysique» s'est enrichi de «la morale» et de «l'éducation», avec le même éditeur-directeur: Pierre de Lacretelle, dit l'aîné (1751-1824), assisté de son frère Jean dit Lacretelle le jeune (1766-1855). 
Metamorfosi dei

Lumi 10.

L'emergenza

del

sentimento de la Pharmacie. $9^{\circ}$. La Biographie médicale, c'est-à-dire, les vies des Médecins célèbres, avec des notices de leurs ouvrages», T. XI, pp. 413D14 => 422G29: «PASSIONS, s. f. pl. (Physiolog., philosophie.)»; T. XI, pp. 422G30 => 435D49: «PASSIONS (Médecine pratique Ẽ Hygiène)».

Cet inventaire nous donne bien deux grands groupes de «PASSION/S». Suivons l'ordre chronologique et examinons d'abord celui du dictionnaire de «Logique, Métaphysique et Morale» de 1788-1791, c'est-à-dire de l'époque préet primo-révolutionnaire, d'avant les mutations radicales initiées en l'An II/1793-4. L'article «PASSION», sans S $^{5}$, de Lacretelle l'aîné ${ }^{6}$ comprend quatre points ayant chacun un titre en italique. Les voici, donnés les uns à la suite des autres pour avoir une vue d'ensemble et essayer d'en percevoir la logique:

"[1] Explication particulière de tous les changemens qui arrivent au corps Ẽ à l'âme dans les passions. [92G1 => 96D6].

«[2] Que les plaisirs $\mathcal{E}$ les mouvemens des passions nous engagent dans l'erreur au regard du bien, Ẽ qu'il faut y résister sans cesse. Manière de combattre le libertinage. [96D7 => 99D42].

"[3] Des passions en particulier, Ė en général de la manière de les expliquer, $\mathcal{E}$ les erreurs dont elles sont la cause [99D43 => 102D3].

«[4] Que toutes les passions se justifient, E des jugemens qu'elles font pour leur justification. [102D4 => 105D4]».

Explicitation. Le point 2 nous confirme que cet article analytique $^{7}$ de la PASSION au singulier considère les «passions» dans la perspective de la finalité traditionnelle, voire traditionaliste chrétienne de tout traité des passions: leur maîtrise pour notre Salut. Preuve de cela: cette phrase de la conclusion de ce point 2, quasi-pascalienne:

5. Curieux, ce singulier, car, usuellement, PASSION au singulier renvoie à la seule passion sublimement acceptable: celle du Christ.

6. Lacretelle l'aîné est en effet l'auteur de tout ce dictionnaire de «Logique et Métaphysique». Mais comme il l'a conçu dès le départ comme «une compilation de tous les meilleurs traités, dissertations ou morceaux sur la Métaphysique et la Logique» (Tome I [1786], «Discours préliminaire», p. xvi), les articles sont autant de morceaux choisis empruntés à des auteurs d'avant lui, référencés le plus souvent, mais pas très précisément. D'où notre long travail de repérage des sources de ces morceaux choisis!

7. Car dans ce même «Discours préliminaire», à la même page, Lacretelle l'aîné se réclame de Condillac. 
L’Encyclopédie

Méthodique

des passions

au début

du XIX siècle
Il est clair, par les choses que nous venons de dire, que l'objet de nos passions n'est point notre bien: que nous ne devons en suivre les mouvemens, que pour la conservation de notre vie: que le plaisir sensible est à l'égard de notre bien, ce que nos sensations sont à l'égard de la vérité; \& que de même que nos sens nous trompent touchant la vérité, nos passions nous trompent touchant notre bien: que l'on doit se rendre à la délectation de la grace; parce qu'elle nous porte avec évidence à l'amour du vrai bien, qu'elle n'est point suivie des reproches secrets de la raison, comme l'instinct aveugle \& le plaisir confus des passions ${ }^{8}$.

Les «sens, sensations» et, sans doute, tout ce qui renvoie à ce qui ne relève pas de la raison et de la grâce est condamnable et condamné. Sauf l'instinct de survie; quand même!

Pour ce qui est des trois autres points qui encadrent ce point 2 , ils constituent en quelque sorte une analyse phénoménologique des passions.

Le 1 expose la nature des passions et leur étiologie physiologique. Elles sont constituées de «sept choses»: 1/ du regard que chacun porte sur un objet ou sur un être; 2/ du mouvement d'approche ou de rejet vis-à-vis de cet objet ou de cet être; 3 / du sentiment d'amour ou d'aversion à l'égard de cet objet ou de cet être; 4/ d'un trouble physiologique; 5/d'un trouble psychologique; 6/ du sentiment né de ces troubles; $7 /$ du sentiment de satisfaction de s'abandonner à sa passion, y compris dans la haine.

Et elles ont pour explications anatomo-physiologique - empruntées aux auteurs du $\mathrm{XVII}^{\mathrm{e}}$ et en particulier à Descartes et à Gassendi via Barbier - l'ébranlement du cerveau et du cour à la vue de l'objet passionnant, qui déclenche le mouvement des «esprits animaux [qui] en se répandant dans les nerfs qui vont au foie, à la rate, au pancréas, \& généralement à tous les viscères, les agitent \& les secouent, \& expriment par ces agitations les humeurs que ces parties conservent pour les besoins de la machine» ${ }^{9}$.

8. T. II, p. $99 \mathrm{D} 21=>34$.

9. T. II, p. $94 \mathrm{D} 46=>51$. Pour Gassendi, voir ci-dessus la note 1 et, pour le texte en français, F. Barnier, Abrégé de la philosophie de Gassendi, Anisson \& Posuel, Lyon 1678, T. 6, pp. 386-388. Pour Descartes, voir Les Passions de l'âme, 1649, art. 7 à 12. Pour la mise en situation de l'étiologie anatomo-physiologiste de l'un et de l'autre, l'article de Carole Talon-Hugon cité à la même note 1 dit l'essentiel, p. 38: «Ainsi, Gassendi ne partage pas la modernité de ceux qui comme Descartes [Hobbes et 
Metamorfosi dei

Lumi 10.

L'emergenza

del

sentimento

Le point 3, quant à lui, constitue le passage obligé de tous les traités des passions: leur inventaire. Où Descartes en voyait six primitives (art. 69): l'admiration, l'amour, la haine, le désir, la joie et la tristesse, Lacretelle l'aîné n'en compte plus que trois: le désir, la joie et la tristesse auxquelles toutes les autres «se peuvent rapporter». "Car les mots d'espérance, de crainte, de hardiesse, de peur, de honte, d'impudence, de colère, de pitié, de moquerie, de repentir, de regret, enfin les noms de toutes les autres passions, sont dans l'usage ordinaire des abregemens de plusieurs termes, par lesquels on peut expliquer en détail tout ce que les passions enferment» ${ }^{10}$.

Quant au point 4 de cet article PASSION, il est consacré à ce qui justifie la réprobation des passions; à savoir leur perfidie qui fait que toute passion s'auto-justifie et tend ainsi à se continuer. Et Lacretelle l'aîné de prendre comme exemple l'amour. Mais l'amour de quoi? On pourrait s'attendre à l'amour de l'autre sexe. Non! C'est l'amour du savoir, l'amour des mots, l'amour de la langue, l'amour des sciences, l'amour de l'érudition que dénonce Lacretelle l'aîné. Tous ces amours, tout cet amour n'aboutit à rien sinon qu'à une immense vanité fondée sur la reproduction de lui-même. Ce qui amène les lignes suivantes qui synthétisent la conception des passions de notre auteur: produits trompeurs de mécanismes anatomo-physiologiques.

Les passions ne corrompent pas seulement l'imagination \& l'esprit en leur faveur elles produisent encore dans le reste du corps toutes les dispositions nécessaires à leur conservation. Les esprits [sous-entendu animaux] qu'elles agitent ne s'arrêtent pas dans le cerveau, ils se répandent, comme j'ai dit ailleurs [voir page précédente], dans toutes les parties du corps, dans celles dont les mouvemens sont visibles, \& dans celles qui donnent la vie \& la nourriture à toutes les autres, par des mouvemens invisibles. Ils se répandent principalement dans le cœur, dans le foie, dans

Spinoza], ont entendu faire une science des passions. Cette question appartient donc à la physique, mais n'en relève pas». Distinction subtile! Pour «les esprits animaux», rappel de leur définition par le Furetière (1690): «En termes de Medecine, se dit des atomes légers \& volatils, qui sont les parties les plus subtiles des corps, qui leur donnent le mouvement, \& qui sont moyens entre le corps \& les facultés de l'ame, qui lui servent à faire toutes ces operations».

10. T. II, p. $100 \mathrm{G} 51=>57$. 
L’Encyclopédie

Méthodique des passions au début du XIX siècle la ratte $[s i c]$, \& dans les nerfs qui environnent les principales artères. Enfin ils se jettent dans les parties telles [= quelles?] qu'elles soient, lesquels peuvent fournir les esprits nécessaires à la conservation de la passion qui domine. Mais lorsque ces esprits se répandent ainsi dans toutes les parties du corps, ils y détruisent peu à peu tout ce qui peut résister à leurs cours \& il y font enfin un chemin si glissant $\&$ si rapide que le plus petit objet nous agite infiniment, \& nous porte par conséquent à former des jugemens qui favorisent les passions. C'est ainsi qu'elles s'établissent et se justifient ${ }^{11}$.

Après cette présentation dénonciatrice des passions au tome 2, le lecteur peut se demander quel peut bien être, pour lui-même et l'auteur, l'intérêt de l'article PASSIONS du tome 4 de ce dictionnaire de «Logique et Métaphysique» devenu de «Logique, Métaphysique et Morale»? Réponse: peut-être enfoncer le clou de la malfaisance trompeuse des passions et donc de la nécessité de les maîtriser, ne serait-ce qu'en apparence! En utilisant pleinement l'instrument assumé de la "compilation» - déjà évoquée - d'auteurs de référence en matière d'analyse morale. De moralistes. Voici ce que cela donne dans le détail de cet article de Lacretelle de ce tome 4.

Une introduction ${ }^{12}$. Qui est purement et simplement le copier-coller des quatre premières colonnes de l'article «PASSIONS (Philos. Logique, Morale.)» de l'Encyclopédie $e^{13}$, inventoriant les quatre grandes sources de passions:

" $1^{\circ}$ Les plaisirs $\mathcal{E}$ les peines des sens»;

« $2^{\circ}$ Les plaisirs de l'esprit ou de l'imagination»;

«3 ${ }^{\circ}$ L'amour de nous-mêmes»;

« $4^{\circ}$ Le bonheur ou le malheur d'autrui».

11. T. II, p. $105 \mathrm{G} 5=>28$.

12. T. IV, pp. $1 G 41=>3$ D58.

13. T. IV, p. 3D58: «Anc. Enc.»= Encyclopédie, tome XII (1765), pp. 142G22 => 143D74. Comme déjà dit, l'article est sans doute de Jaucourt qui indique lui-même (p. 144G6=>9) que, pour ces quatre premières colonnes, il «a fait trop d'usage d'un petit mais excellent ouvrage sur la théorie des sentimens agréables, pour ne pas lui rendre toute la justice qu'il mérite». Il s'agit de:J.L. Levesque de Pouilly, Théorie des sentimens agréables. Où après avoir indiqué les règles que la Nature suit dans la distribution du plaisir, on établit les principes de la Théologie naturelle et ceux de la Philosophie morale, Chez de Bure père, Genève 1747, p. 239. De la confrontation avec cet ouvrage des quatre colonnes de Jaucourt, il résulte que «l'usage» que celui-ci en a fait n'a rien à voir avec le copier-coller de Lacretelle. C'est plutôt une source d'inspiration. 
Metamorfosi dei

Lumi 10.

L'emergenza

del

sentimento

Une première partie présentant les différents types de passions en cinq points:

[1] «Des passions qui tirent leur origine du corps» ${ }^{14}$.

[2] «Des passions qui ont leur source dans un tour, ou disposition particulière de l'imagination ${ }^{15}$;

[3] «Des passions insociables, ou avec lesquelles il répugne au spectateur de s'associer ${ }^{16}$;

[4] «Des passions, sociables, ou avec lesquelles s'associe volontiers le spectateur ${ }^{17}{ }$;

[5] «Des passions qui se bornent à nous-mêmes, ou qui ont pour objet notre intérêt personne» ${ }^{18}$.

En confrontant simplement les titres de ces cinq parties avec les quatre points de l'introduction, on voit que Lacretelle est quelque peu redondant dans sa typologie des passions. En fait, il semble que ladite typologie soit un passage obligé de la classification des passions: celles venant du corps; celles venant de l'esprit; celles venant des rapports positifs ou négatifs aux autres; celles venant de notre intérêt. C'est celle de l'Encyclopédie qui, peut-être, en est à l'origine. C'est celle aussi de l'autre source avouée de Lacretelle à la fin de ces cinq parties: "(Théorie des sentimens moraux)», c'està-dire la Théorie des sentimens moraux d'Adam Smith ${ }^{19}$ d'où viennent quasi mot pour mot et les titres et le contenu des 5 points énoncés ci-dessus. Ce sont ceux de l'introduction et des chapitres I, II, III, IV et V de la section II, «Des degrés où les différentes passions sont compatibles avec la convenance. ${ }^{20}$, de cet ouvrage. Le sens général est celui du relativisme social des passions qui sont regardées «comme bien ou mal-faisantes, justement dans la proportion que les hommes sont plus ou moins disposés à sympathiser avec elles» ${ }^{21}$. Ce qui veut dire qu'elles ne sont plus malfaisantes en elles-mêmes. Entre le tome 2 (1788) et le tome 4 (1791

14. T. IV, pp. $4 \mathrm{G} 35=>6 \mathrm{G} 21$.

15. T. IV, pp. $6 \mathrm{G} 22=>7 \mathrm{D} 15$.

16. T. IV, pp. $7 \mathrm{D} 16=>10 \mathrm{G} 5$.

17. T. IV, pp. $10 \mathrm{G} 6=>11 \mathrm{G} 2$.

18. T. IV, pp. $11 \mathrm{G} 3=>12 \mathrm{D} 6$.

19. Traduction nouvelle par l'Abbé Blavet, Valade, Paris 1775,

20. Théorie des sentiments moraux, Valade, Paris 1774, pp. 52-95. Correspondant donc aux pp. 4G1 $\Rightarrow 12$ D6 de cet article «PASSIONS» de Lacretelle l'aîné.

21. T. IV, pp. $4 \mathrm{G} 32=>34=$ Théorie des sentiments moraux, t. 1, p. 52 . 
L'Encyclopédie Méthodique des passions au début du XIX ${ }^{\mathrm{e}}$ siècle une laïcisation des passions s'est opérée; elles ne sont plus jugées mauvaises en fonction du salut chrétien, mais bonnes ou mauvaises en fonction de leur convenance sociale) ${ }^{22}$.

Les deuxièmes et troisièmes parties sont plus traditionnelles, constituées d'aphorismes ou de maximes moraux empruntés à deux grands moralistes.

La deuxième partie ${ }^{23}$ indique finalement comme source: «(Connoissance de l'esprit humain)», c'est-à-dire l'Introduction à la connaissance de l'esprit humain, suivie de Réflexions et de Maximes de Luc de Clapiers de Vauvenargues ${ }^{24}$. Précisément, Lacretelle l'aîné en recopie une partie du «Livre II. Des Passions» ${ }^{25}$. Et de celui-ci il retient soixante-sept aphorismes tirés de dix-huit têtes de chapitres non citées par lui ${ }^{26}$, en laissant de côté les trois dernières: «De l'Estime, du Respect, et du Mépris»; «De l'Amour des objets sensibles» et surtout; «Des Passions en général» ${ }^{27}$.

Que tirer de tous ces aphorismes? Que les passions sont un donné de l'être humain qu'il faut prendre pour ce qu'elles sont: un ressort des actions humaines. Et Lacretelle l'aîné de reprendre de Vauvenargues la formule de «Loke» [sic]: «les passions roulent sur le plaisir \& la douleur: c'en est l'essentiel \& le fond ${ }^{28}$, leurs variations venant simplement des différenciations entre les êtres humains, entre les individus. Leur malfaisance pour le salut de ceux-ci, oubliée! Les passions sont entrées dans le champ de la science de l'homme à construire.

La troisième partie est constituée de tous les aphorismes du chapitre ou de la partie IV «Du Cœur» des Caractères de La Bruyère, livrés tels quels, sans numérotation ${ }^{29}$. Ce qui fait

22. Au départ, peut-être grâce à l'Encyclopédie et Jaucourt.

23. T. IV, pp. $12 \mathrm{D} 7=>17 \mathrm{D} 36$.

24. Briasson, Paris 1746, p. 384.

25. Pp. $43 \Rightarrow 91$ de l'édition de 1746 ou pp. $47 \Rightarrow 83$ de l'édition de 1781 , correspondant donc aux pp. 12D7 => 17D36 du tome IV indiquées ci-dessus à la note 22. 26. Estimant que les donner une à une avec l'équivalence dans notre dictionnaire de «Logique, Métaphysique et Morale» de la Méthodique allongerait un peu notre propos, nous tenons à la disposition du lecteur le tableau de cette équivalence; il suffit de nous le demander sur danielteysseire@gmail.com.

27. Ce qui lui fait passer à côté de cette notation de Vauvenargues, p. 88, promise à un bel avenir: «Les passions s'opposent aux passions, \& peuvent se servir de contre-poids».

28. T. IV, p. 12D7 = Vauvenargues, ouv. cité, p. 43.

29. T. IV, p. 17D 37 => 21D14 (= fin de l'article «PASSIONS» avec la mention de 
Metamorfosi dei

Lumi 10.

L'emergenza

del

sentimento

que les différents paragraphes d'un même aphorisme chez celui-ci deviennent avec Lacretelle autant d'aphorismes séparés; au lecteur de s'y retrouver, comme, par exemple, dans le long aphorisme sur le gouvernement de soi-même ${ }^{30}$. À la vue de l'ensemble de cet article «PASSIONS», cette reproduction du chapitre «Du Coeur» des Caractères du moraliste français par excellence de l'époque classique sert à traiter le sujet de la passion un peu laissée de côté jusquelà par ledit article; celle de l'amour. Ce qui veut dire que, visiblement, les affres et les extases de l'amour préromantique des Lumières de la seconde moitié du XVIII ${ }^{\mathrm{e}}$ siècle ne semblent pas avoir touché Lacretelle l'aîné au point de le pousser à en faire l'analyse - même par morceaux choisis interposés, de Rousseau par exemple - dans le dictionnaire de «Morale» de la Méthodique dont il a la charge!

Dans ces conditions et étant donné tout ce que nous en avons vu, la conclusion que le lecteur doit tirer de cet article «PASSIONS», n'est-elle pas dans cet aphorisme des Caractères recopié par Lacretelle l'aîné:

Toutes les passions sont menteuses, elles se déguisent autant qu'elles le peuvent aux yeux des autres, elles se cachent à elles-mêmes. Il n'y a point de vice qui n'ait une fausse ressemblance avec quelque vertu, \& qui ne s'en aide $^{31}$ ?

Ainsi, les passions sont bel et bien entrées dans le champ de la science de l'homme, mais dans un bien mauvais état! Telle est la leçon dudit article du dictionnaire de «Logique, Métaphysique et Morale» de la Méthodique.

2. La science des passions dans le «Dictionnaire de médecine» de l'E.M.: inventaire analytique détaillé et usage thérapeutique. Avec le dictionnaire «Médecine» tout change. Les passions sont l'objet d'une autre approche; celle d'une science, à tout le moins d'un savoir qui veut au minimum rendre compte

la source de cette troisième partie: «(Caratère [sic] de la Bruyère)». Correspond aux pages 133 => 144 de la belle édition de Coste des Caractères, Hochereau et Panckoucke, Paris 1765.

30. Caractères, ed. citée, pp. 141-143 correspondant aux pp. 20D10 $\Rightarrow 21$ G13 de notre T. IV.

31. T. IV, p. $21 \mathrm{G} 14=>18$, correspondant à un aphorisme, le $69^{\text {ème }}$, de la p. 143 de l'éd. des Caractères référencée ci-dessus. 
L'Encyclopédie Méthodique des passions au début du XIX ${ }^{\mathrm{e}}$ siècle

de cette réalité humaine essentielle qu'elles constituent. Sans aucunement se préoccuper des connotations, positive et surtout négatives, qui lui sont attachées. Les passions sont inhérentes à la condition humaine, peu importe pour quelle signification qui les transcende, mais de quelle manière, telle est la question. Question congruente au phénoménalisme des Lumières. Une fois répondu à cette question, vient l'autre question typique des Lumières: puisque les passions humaines sont là et bien là, qu'est-ce que l'on peut en faire qui puisse être utile à l'être humain et à l'humanité? Voilà ainsi posé les deux points de l'article PASSIONS ${ }^{32}$.

D'abord, en faire un inventaire ${ }^{33}$. Dont la présentation nous est facilitée par le fait que l'auteur des deux entrées de cet article est le directeur-éditeur (au moins depuis 1821, si ce n'est depuis 1815) de l'ensemble du dictionnaire de «Médecine [etc.]», et l'auteur des entrées «MEDECINE MENTALE» et «MEDECINE MORALE» ${ }^{34}$ de l'article général «MEDECINE»: Moreau de la Sarthe ${ }^{35}$. Or, dans cette entrée «MEDECINE MORALE» se trouve une «Table synoptique des objets que comprennent la médecine morale et la physiologie des passions» - nous y voilà - qui nous livre son inventaire général des passions ${ }^{36}$. Voyons ce tableau ci-joint.

32. C'est notre corpus énoncé au début de notre propos: dictionnaire de «Médecine», Veuve Agasse, Paris 1824:

T. XI, pp. 413D14 => 422G29: «PASSIONS, s. f. pl. (Physiolog., philosophie» - c'est la partie inventaire); T. XI, pp. 422G30 => 435D49: «PASSIONS, (Médecine pratique Eீ Hygiène)»- c'est la partie utilité.

33. Avant l'inventaire proprement dit, Moreau de la Sarthe développe une longue introduction (T. XI, p. 413D15 $=>416 \mathrm{G} 12)$ en trois points: $1 /$ définition et causalité générale psycho-physiologique (p. 414G36 $\Rightarrow$ 43) des passions dans le cadre de l'unicité physique/morale de l'homme; 2/ historicité des passions tant au plan individuel qu'au plan social, en fonction du progrès de l'individu et de la civilisation; 3 / typologie générale des passions et de leurs effets organiques et manifestes.

34. T. IX (1816), pp. 136D $\Rightarrow$ 219D et pp. 393D $\Rightarrow$ 451D.

35. Sur Moreau de la Sarthe, voir Véronique Signoret, Louis-Jacques Moreau dit de la Sarthe (1771-1826), Présentation d'un Idéologue méconnu et de son æeuvre, Créteil Université de Paris XII-Val de Marne, 1994, p. 314., et notre contribution "'Le Dictionnaire de Médecine' de l'Encyclopédie méthodique: la méthodicité à l'œuvre» dans l'ouvrage collectif à paraître aux éditions Garnier, ss. la dir. de Martine Groult et Luigi Delia. Nous y soulignons, entre autres, l'attachement de Moreau de la Sarthe au monisme de son maître Cabanis, mettant l'accent sur l'unité psycho-physiologiste ou physio-psychologiste de l'être humain. C'est d'ailleurs ce monisme qui est à l'œuvre dans tout cet inventaire des passions.

36. T. IX (1816), p. 396. 
DES OBJETS QUE COMPRENAENT LA MÉDECINE MOAALE ET LA PHXSIOLOGIE DES PASSIONS

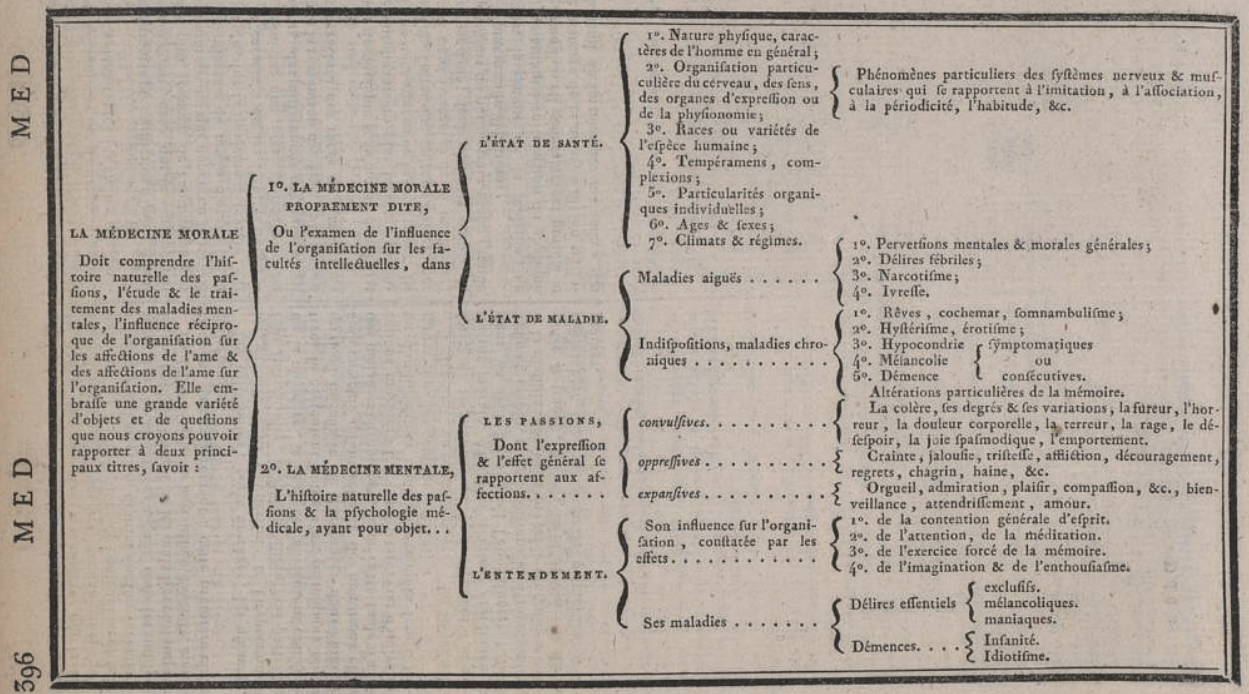

Toutes les passions inventoriées sont réparties en trois grands groupes selon l'effet physio-psychologique dominant. Et chacun de ces grands groupes est analysé selon le plan suivant: une présentation des manifestations physiques et physiognomoniques ${ }^{37}$ d'une ou des passions du groupe considéré; une analyse de type médical des «signes volontaires» et surtout «involontaires» ou organiques des passions de chaque groupe; la mention d'une ou plusieurs œuvres d'art illustrant une ou des passions des divers groupes - manifestation de la formation et de la culture classiques des médecins de cette génération des Lumières fulminantes, autrement dit de l'époque de la Révolution.

Voici ce que cela donne.

37. En se servant - ce qui est notable - du texte de la conférence sur «L'Expression des passions» donnée les 7 avril et 5 mai 1668 par le peintre Le Brun à l'Académie royale de peinture et de sculpture, éditée, en 54 pages avec gravures, à Amsterdam et Paris en 1698. Voir A. Merot, Les conférences de l'Académie royale de peinture et de sculpture au XVII siècle, Ecole nationale supérieure des Beaux-Arts, Paris, 1996, pp. 145-162. 
L’Encyclopédie

Méthodique

des passions

au début

du XIX siècle

$1 /$ Pour les «convulsives ${ }^{38}$,

[Elles] se montrent, dans quelques cas, avec toutes les apparences d'une attaque de nerfs, d'un accès de délire ou de rage. La colère \& la fureur, auxquelles cette remarque s'applique d'une manière particulière, sont toujours annoncées par des regards enflammés, par un œil étincelant, par la rougeur ou quelquefois par une pâleur effrayante de la face, par la décomposition \& le renversement de tous les traits du visage.

Voilà pour les manifestations physiognomoniques. Suit l'énoncé des signes organiques internes.

L'action du cœur \& celle des muscles sont ordinairement augmentées dans le développement de ces passions, lorsque leur violence n'a pas dérangé l'équilibre des organes. Le cerveau est également excité, \& devient capable de la plus violente réaction. Du reste, les signes volontaires sont en petit nombre: ce sont toutes les actions pour menacer, attaquer, combattre. Presque tous les autres signes sont indépendans de la volonté, \& entièrement corporels \& automatiques. Le cercle sous-orbitaire s'enfle, devient livide. Les muscles du nez, des joues, se gonflent aussi: la bouche demeure ouverte; les veines, les muscles du cou se dessinent \& paroissent tendus. Les cheveux sont agités, en désordre ${ }^{39}$.

Quant aux ouvres d'art illustrant ces passions convulsives, Moreau de la Sarthe en donne cinq: le «Laocoon», «la tête du comte Eugolino par Reynolds» et trois tableaux du Poussin, la «Résurection [sic] d'une jeune fille au Japon, par saint François d'Assise», l' «Enlèvement des Sabines» et la «Femme adultère [sur lequel] l'homme placé derrière le Christ offre tous les traits de la colère ${ }^{40}$.

2/ Dans les «oppressives» ${ }^{41}$,

on observe une angoisse plus ou moins vive, une grande oppression, la langueur ou le resserrement de la peau; la foiblesse ou la concentration du pouls; la décoloration de la face, l'alongemenr ou l'affoiblissement des traits du visage, en un mot tous les signes d'une diminution ou d'une concen-

38. T. XI, pp. $416 \mathrm{G} 123=>417 \mathrm{D} 12$.

39. T. XI, pp. 416 G28 $\Rightarrow 52$.

40. T. XI, p. $416 \mathrm{D} 21 \Rightarrow 23$.

41. T. XI, pp. 417D13 => 419G19. 
Metamorfosi dei

Lumi 10.

L'emergenza

del

sentimento tration dans les forces vitales. Les signes involontaires, sans appartenir exclusivement à ces passions, y dominent, surtout dans l'affliction subite, dans la haine, la jalousie, dans l'inquiétude portée à son comble, afflictions qui, par la force de leur expression, se rapprochent des passions convulsives ${ }^{42}$.

Et Moreau de la Sarthe de prendre comme exemple le personnage de Mithridate dans la tragédie éponyme de Racine, dont la jalousie «dissimulée \& concentrée» se traduit par une «pâleur livide et sombre» du visage qui, à l'aveu de Monime, prend «des teintes les plus enflammées [par] le trouble, la fureur, le désespoir» ${ }^{43}$.

3 / Pour les «expansives» ${ }^{44}$, au premier rang desquelles «doivent être placées la joie \& l'amoun»,

les caractères généraux [en] sont l'afflux du sang artériel dans les vaisseaux capillaires du visage; l'épanouissement des traits; la contraction régulière $\&$ douce des muscles, qui augmente le diamètre transversal de la face. Nous devons faire remarquer d'une manière plus particulière, que les muscles zygomatiques ne jouent pas un rôle moins important dans l'expression de ces passions, que celui des muscles frontaux $\&$ sourciliers dans l'expression des passions oppressives ${ }^{45}$.

Fait notable: c'est pour ce type de passions que Moreau de la Sarthe est le plus prolixe en illustrations artistiques ${ }^{46}$. Pour le mépris, le Christ à la colonne du Titien. Pour l'ironie, le jeu contemporain de Talma dans Nicomède de Corneille. Pour l'espérance, le Saint Jérome du Dominiquin et les Trois vertus théologales de Raphaël. Pour l'amour maternel, érigé donc en passion, la Sainte Famille, le Sommeil de l'enfant Jésus et la Madonna della Sedia de Raphaël, et le portrait de «la reine de Médicis» de Rubens ${ }^{47}$. Pour la compassion, la Mort de Saphyre du Poussin. Pour l'attention, l'Ecole d'Athènes de Raphaël et

42. T. XI, p. $417 \mathrm{D} 19=>31$.

43. T. XI, p. $418 \mathrm{G} 25=>36$.

44. T. XI, pp. $419 \mathrm{G} 20=>421 \mathrm{D} 13$.

45. T. XI. p. 419 G21 $\Rightarrow 31$.

46. Raison pour laquelle nous renonçons à donner la référence des lignes précises où sont mentionnées les œuvres. Elles se trouvent dans la partie indiquée à la note 43.

47. T. XI, p. 420G29. Il s'agit sans doute de La Naissance du Dauphin à Fontainebleau. A moins que ce soit Marie de Médicis, reine mère de France. En tous les cas, pour Moreau de la Sarthe «l'une des plus belles expressions composées \& mixtes, que la peinture ait jamais pu atteindre». 
L’Encyclopédie Méthodique des passions au début du XIX siècle
Saint Bruno prêchant la théologie à Reims d'Eustache Le Sueur. Pour la pudeur, la Sainte Famille de Raphaël et Suzanne aux bains de Jean-Baptiste Santerre. C'est dans ce développement sur la pudeur, sentiment manifestement élargi en passion ${ }^{48}$, que Moreau de la Sarthe livre des considérations ethnographiques sur la pudeur des «nègres» dont la peau subit alors «une diminution subite dans le ton noirâtre». La preuve en est donnée par le récit d'un «voyageur trop éclairé pour avoir été un observateur inexact et infidèle» racontant comment une esclave «Madegasse» [= Malgache] nommée Maria avait $\mathrm{eu}$ «son teint de jais» prendre une «nuance rougeâtre», quand sa pudeur avait été alarmée à dessein par la demande du voyageur de voir sa "gorge» ${ }^{49}$.

La conclusion de cette partie inventaire est constituée par un refus et une proclamation. Le refus est celui de toute théorie voulant localiser un siège précis des passions, et en dernier lieu celle de Gall sur les localisations cervicales différenciées des passions. La proclamation est la suivante:

Les considérations qui viennent d'être exposées \& la description rapide des principaux effets des passions sur l'organisation en général, \& sur les traits du visage en particulier, forment, sans doute, la partie principale de l'histoire physiologique des passions. Ce qui concerne leurs causes \& leurs sources diverses, leurs développemens, la liaison secrète qui en rattache le plus grand nombre aux besoins, aux penchans, aux dispositions intellectuelles ou primitives, aux spécialités [= spécificités] organiques, que l'on appelle des idiosynchrasies, appartiennent aussi à cette histoire ${ }^{50}$.

Ce qui revient à confirmer explicitement que les passions sont des objets de science comme les autres et donc, à ce titre de réalités objectives, des instruments utiles à l'humanité.

Ainsi, après avoir fait l'inventaire des passions en fonction de leurs manifestations physio-psychologiques, il convient de les connaître plus organiquement et, surtout, d'en voir leurs effets, utiles ou non. C'est l'objet de la deuxième entrée de l'article PASSIONS, la plus longue, vingt-sept colonnes

48. Ce qui ne doit pas étonner, puisque dès le départ les passions ont été définies très généralement comme «affections de l'âme, modifications de la sensibilité» ( $\mathrm{T}$. XI, p. 413D17/18).

49. T. XI, p. $421 G 23=>51$.

50. T. XI, p. $421 \mathrm{D} 14=>25$. 
Metamorfosi dei

Lumi 10.

L'emergenza del sentimento contre dix-sept et demie ${ }^{51}$. Elles se divisent en quatre parties appuyées sur des observations cliniques au nombre de vingtquatre, certes plus ou moins précises, mais des observations cliniques tout de même qui témoignent du triomphe de la clinique, puisque donc elle est présente dans un dictionnaire général de médecine.

La première est un inventaire pathologique qui, en quelque sorte, prend la suite de l'inventaire analytique ${ }^{52}$. Dans le cadre de l'examen général de «l'influence réciproque des organes sur les passions \& celle des passions sur l'état des organes ${ }^{53}$, ladite première partie considère le premier sens de cette réciprocité; celui du physique sur le moral: l'influence des pathologies organiques sur les passions. Sont donc examinées successivement les influences suivantes propres à créer ou modifier les passions.

1/ Celle du tempérament, ou lymphatique, ou sanguin, ou bilieux sur la constitution des passions, le sanguin ayant des «passions vives» et le bilieux des «passions plus profondes», le mélancolique étant laissé de côté ${ }^{54}$.

2/ Celle du régime, Rousseau étant cité comme ayant eu «l'idée d'un régime qui aurait rendu la pratique de la vertu plus douce \& plus facile» - idée réalisée «d'une manière péremptoire» pour «l'amendement des criminels» dans «les établissements philanthropiques de New-Yorck \& Philadelphie ${ }^{55}$.

3/ Celles, «habituelles \& permanentes», du sexe, de l'âge, «du climat \& des variétés nationales» ${ }^{56}$, tellement connues que Moreau de la Sarthe ne s'y attarde pas.

4/ Celle «des complexions héréditaires» morbides dont le sagace «M. Esquirol» a remarqué «avec raison» qu'elles sont à l'origine des troubles du comportement qui ont précédé «l'interdiction ou la réclusion» des aliénés ${ }^{57}$.

5/ Celle de certaines «dispositions accidentelles \& passagères de l'organisation» dans lesquelles on trouve, dans

51. Rappel. «PASSIONS (Médecine pratique Ẽ Hygiène), T. XI, pp. 422G30 => 435D49.

52. T. XI, pp. $422 \mathrm{G} 43 \Rightarrow 426 \mathrm{G} 14$.

53. T. XI, p. $422 \mathrm{G} 34$ et 35 .

54. T. XI, p. $422 \mathrm{D} 28=>42$.

55. T. XI, p. $422 \mathrm{D} 54=>423 \mathrm{G} 2$.

56. T. XI, p. $423 \mathrm{G} 14$ et 15 .

57. T. XI, p. $423 \mathrm{G} 12=>\mathrm{D} 16$. 
L'Encyclopédie

Méthodique des passions au début du XIX siècle

l'ordre, l'hypocondrie, le spasme intestinal, les troubles passagers, la crise nerveuse, la grossesse, les maladies chroniques gastro-intestinales, la phtisie, la puberté et la menstruation, les changements climatiques, les médicaments et certaines substances, comme les «boissons aromatiques \& spiritueuses, le café \& le vin de Champagne, qui éveillent si doucement la pensée, \& qui donnent tant de grâce, un mouvement si facile à l'imagination», et la fièvre créatrice ${ }^{58}$.

La deuxième partie de cet inventaire pathologique s'occupe de l'influence inverse, celle du moral sur le physique, celle des passions sur l'organisme ${ }^{59}$. Elle peut s'exprimer en un seul mot: «commotions», "plus ou moins fortes, plus ou moins vives ${ }^{60}$. Qui touchent différents organes, «le cœur, le diaphragme \& les viscères de la région précordiale ${ }^{61}$ ou d'ailleurs, plus ou moins fortement selon la nature physiologique de l'individu et la nature de la passion, convulsive, oppressive ou expansive. Ainsi «la colère \& les passions convulsives en général, agissent principalement sur la circulation \& sur l'état $\mathrm{du}$ foie ${ }^{62}$, tandis que la joie, passion expansive, «augmente l'action des vaisseaux capillaires, la respiration cutanée, la force musculaire» ${ }^{63}$, alors que «la tristesse \& les affections oppressives en général [...] paraissent agir plus particulièrement sur les fonctions de la peau, sur la digestion \& sur la nutrition, occasionnant, à la longue, le dépérissement et une langueur générale» ${ }^{64}$. Suit le tableau très sombre des pathologies organiques causées par ces passions oppressives - les plus mauvaises: «l'inflammation, l'engorgement du foie, du mésentère, du cœur, l'endurcissement, l'hépatisation des poumons, les adhérences à la plèvre, \&c. \&c.» ${ }^{65}$. Et Moreau de la Sarthe d'ajouter que «les passions, dont les effets sur les différents organes \& sur leurs diverses fonctions sont si remarquables, n'exercent pas une influence moins puissante
58. T. XI, pp. $424 \mathrm{G} 1 \Rightarrow 426 \mathrm{G} 14$.
59. T. XI, pp. 426G15 $\Rightarrow$ 429D24.
60. T. XI, p. 426G26-28.
61. T. XI, p. 426G58-59.
62. T. XI, p. 427D26-28.
63. T.XI, p. $427 \mathrm{D} 36$ et 37.
64. T. XI, p. $427 \mathrm{D} 43 \Rightarrow 45$.
65. T. XI, p. $428 \mathrm{G} 24=>27$. 
Metamorfosi dei

Lumi 10.

L'emergenza del sentimento

sur le cerveau lui-même et l'entendement» ${ }^{66}$. À preuve, les passions «qui se rapportent à des intérêts politiques ou religieux» ${ }^{67}$ qui suscitent l'enthousiasme produisant «un recueillement qui absorbe toute l'attention, tout l'intérêt, \& qui suspend ainsi les rapports du cerveau avec les divers genres d'irritation ou d'impression externe ou interne» ${ }^{68}$. C'est par ce modèle de perturbation obsessionnelle du cerveau par une ou des passions que l'on peut expliquer à la fois toute création artistique et intellectuelle et l'aliénation mentale:

Ainsi, tantôt elles [ = les passions] absorbent, elles captivent exclusivement l'intérêt, de telle sorte que ce qui les concerne, devient l'objet d'une idée fixe, \& tantôt elles brisent en quelque sorte pat leur véhémence \& par la soudaineté de leur commotion [on retrouve le terme de départ pour désigner l'effet fondamental des passions], la chaîne des idées, affoiblissent, exaltent l'entendement, \& occasionnent un état de stupeur plus ou moins grave ou un véritable délire ${ }^{69}$.

Avec la troisième partie de cet inventaire organique le lecteur entre dans les considérations sur l'utilité des passions. Comme cette partie a un titre, donnons-le: «Des effets des passions relativement à l'hygiène et à la thérapeutique» ${ }^{70}$. Il indique clairement que les passions sont hygiéniques, puisque «la vie ne peut véritablement se soutenir sans l'ébranlement nerveux, sans l'excitement du cerveau, \& sans l'augmentation du mouvement, que le jeu habituel \& journalier d'un petit nombre de passions entretient dans les organes ${ }^{71}$. Cette «influence vivifiante $\&$ salutaire ${ }^{72}$ des passions est également à l'œuvre dans la thérapeutique de certaines maladies, comme le mal «du pays (nostalgie), quelques hypocondries \& une espèce particulière de consomption ou de spleen, qui porte au suicide» ${ }^{73}$. Et Moreau de la Sarthe de donner cinq observations cliniques ou exemples de guérison de ces maladies par

66. T. XI, p. $428 \mathrm{G} 28=>32$.

67. T. XI, p. 428G32 et 33.

68. T. XI, p. $428 \mathrm{D} 49=>52$.

69. T. XI, p. $429 G 59=>D 7$.

70. T. XI, p. $429 \mathrm{D} 25=>431 \mathrm{G} 43$.

71. T. XI, p. 429D36 $\Rightarrow$ 40. Lignes venant de Bordeu cité en substance.

72. T. XI, p. $431 G 25$.

73. T. XI, p. $429 D 55=>58$. 
L’Encyclopédie Méthodique des passions au début du XIX ${ }^{e}$ siècle le recours à toute passion provoquant «la réaction du système nerveux \& les irradiations du cerveau» ${ }^{74}$ qui en sont l'effet organique. Ainsi, cette «Madame $\mathrm{M}^{* *}$, âgée de quarante à cinquante ans, tombée dans la plus affreuse mélancolie» suicidaire à la suite de revers de fortune dus à la Révolution puis aggravés ensuite, qui fut guérie par ses malheurs mêmes qui la poussèrent à s'investir, dirions-nous aujourd'hui - à se passionner donc "pour une place de concierge dans un hospice [devenue] l'objet de toute son ambition \& de toutes ses espérances» ${ }^{75}$.

Avec la quatrième partie intitulée «De l'influence des passions dans le traitement des maladies ${ }^{76}$, Moreau de la Sarthe semble redondant avec ce qui précède. Il n'en est rien, car elle constitue, avec onze observations cliniques, un véritable élargissement vers une méthode générale de traitement des maladies mentales et donc des malades mentaux. En deux points successifs:

1/ Le traitement moral en général ${ }^{77}$.

2/ Les maladies ressortissant de ce traitement ${ }^{78}$.

Ces deux points étant très exactement explicités et liés à leur jointure par et dans les vingt lignes qui suivent:

Les maladies particulières qui peuvent être plus souvent \& plus utilement combattues par l'effet de certaines passions que par les médicamens les plus énergiques, sont non seulement les maladies mentales, mais encore toutes les affections chroniques, qui rentrent dans la classe des névroses, \& qu'il est possible de guérir, par une perturbation, par une commotion [revoilà le maître-lot] assez profonde pour rompre tout-à-coup une série de mouvemens associés d'une manière vicieuse \& contraire à l'état normal ou habituel des propriétés vitales, qu'il importe de rétablir. Nous plaçons dans cette catégorie, les différentes espèces de spasmes ou de convulsions, capables de se propager par imitation; la danse de Saint-Guy; diverses impotences qui on été confondues avec la paralysie; quelques cas d'épilepsie, d'hystérie, d'hypocondrie; plusieurs fièvres intermittentes, \& divers

74. T. XI, p. $430 \mathrm{G} 59$ et 60.

75. T. XI, pp. $430 \mathrm{D} 53=>431 \mathrm{G} 10$.

76. T. XI, pp. $431 G 44 \Rightarrow 435 \mathrm{D} 48$.

77. T. XI, pp. $431 \mathrm{G} 44 \Rightarrow 433 \mathrm{D} 35$.

78. T. XI, pp. $433 \mathrm{D} 36=>434 \mathrm{D} 50$. 
Metamorfosi dei

Lumi 10.

L'emergenza

del

sentimento symptômes ataxiques que l'on a vus quelquefois se manifester tout-à-coup, au milieu des maladies aigües ${ }^{79}$.

Voilà donc et en quoi consiste cette médecine morale basée sur la «commotion» des passions par des contre-passions, et la liste bien large des pathologies auxquelles elle peut s'attaquer. C'est tout simplement la première psychiatrie largement dimensionnée.

La conclusion de cette quatrième partie élargit encore le propos en évoquant les divers types de médecines mettant en jeu «l'influence du moral sur le physique» ${ }^{80}$. Elle tient lieu en même temps de conclusion générale de l'article, valorisant ainsi la dimension psychologique de la médecine et de sa pratique $^{81}$, synthétisée dans cette citation latine de Boerhaave: «Non semper quaerenda est medicina, ex materie medica \& per pharmacia ${ }^{82}$, la médecine ne doit pas toujours demander à la matière médicale et à la pharmacie.

Pour conclure, je voudrais m'arrêter sur cette quatrième partie consacrée à la médecine morale et-répétons le mot - à cette première psychiatrie ${ }^{83}$. Pour bien la situer historiquement et rendre sinon donner à Moreau de la Sarthe sa place dans la constitution de celle-ci ${ }^{84}$.

Le texte de cette quatrième et même d'un morceau de cette troisième parties de cette deuxième entrée de PASSIONS analysée dans les pages précédentes, n'a pas été écrit un peu avant que ne soit édité en 1824 le tome XI du dictionnaire de «Médecine» de la Méthodique, non! Avec les mêmes observations cliniques, il provient d'un écrit intitulé: Quelques observations sur différentes maladies, A la guérison desquelles les ressources pharmaceutiques n'ont point concouru, suivies de considérations sur la consomption, et de réflexions physiologiques

79. T. XI, p. $433 \mathrm{D} 36=>56$.

80. T. XI, p. $435 \mathrm{G} 18$.

81. Moreau de la Sarthe ayant longuement insisté un peu auparavant sur l'importance des qualités de psychologue que devrait avoir tout médecin et qu'il n'a pas toujours, pour s'attacher «la confiance des malades» et, plus généralement, afin d'assumer cette dimension psychologique des maladies (T. XI, pp. 433G34 => D35).

82. T. XI, p. 435D36-37.

83. Sur cette dénomination, voir encore notre contribution à l'ouvrage référencé plus haut à la note 34 .

84. Dans les ouvrages du binôme français de l'histoire de la psychiatrie, Gladys Swain et Marcel Gauchet, Moreau de la Sarthe semble plutôt inconnu. 
L'Encyclopédie

Méthodique des passions au début du XIX siècle sur l'emploi médical des passions, considérées comme des modifications du système nerveux, susceptibles d'être comparées à l'action des médicamens qu'elles peuvent souvent remplacer avec avantage.

Sa date d'édition, chez Croullebois et Gabon à Paris, est l'An VII de la République. Autrement dit entre l'automne 1798 et l'automne 1799. Rédigé en 1798 et peut-être même avant, il est contemporain du texte de Pinel également édité en l'an VII: "Recherches et Observations sur le traitement moral des aliénés» ${ }^{85}$. Ils s'inscrivent tous les deux, sans doute avec d'autres, dans ce que je n'hésite pas à appeler la révolution intellectuelle - à tous le moins la mutation radicale de l'an $\mathrm{VI}^{86}$. En quoi consiste-t-elle?

En conformité avec la stabilisation institutionnelle que veut incarner le Directoire ${ }^{87}$, toutes les nouvelles institutions intellectuelles, éducatives et sanitaires voulues par la Convention se mettent en place ${ }^{88}$. Parmi elles, au sommet, l'Institut National $^{89}$ avec sa Classe des Sciences morales et politiques tenue par les Idéologistes. C'est là que, en l'an IV et au début de l'an V, c'est-à-dire pendant toute l'année 1796, le médecin philosophe Cabanis lit cinq des six premiers «Mémoires ${ }^{90} \mathrm{de}$ ce qui va devenir ses Rapports du physique et du moral de l'homme, manifeste du monisme de la nature de l'être humain, réalité physio-psychologique ou psycho-physiologique. A partir de quoi la distinction entre le psychologique et le physique n'a plus lieu d'être, en particulier quand il s'agit de traiter des pathologies qui ne sont pas exclusivement des blessures physiques et, bien sûr et surtout, les affections morales.

De cette mutation radicale de l'an VI, Cabanis donc, Pinel et Moreau de la Sarthe sont trois protagonistes importants.

85. Tous deux également publiés an l'an VII dans les Mémoires de la Société médicale d'émulation, séante à l'Ecole de Médecine de Paris: pour l'an VI de la République française. Seconde année - Société dont ils sont tous les deux membres.

86. 22 septembre 1797 - 21 septembre 1798. C'est aussi l'année de la $1^{\text {ère }}$ édition du tome Ier de la Nosographie philosophique de Pinel, à Paris chez Maradan.

87. Date de séparation de la Convention: 4 Brumaire an IV / Lundi 26 octobre 1795.

88. Voir l'important ouvrage de M. S. Staum, Minerva's Message: Stabilizing the French Revolution, McGill-Queen's University Press, Montréal \& Kingston 1996, p. 342.

89. Date de sa séance inaugurale: 15 Germinal, an IV, 4 avril 1796.

90. Dont les deuxième et troisième traitent de l'«Histoire physiologique des sensations» à laquelle se réfère Moreau de la Sarthe à la page 24 de ses «Quelques observations $[\ldots] »$. 
Metamorfosi dei

Lumi 10.

L'emergenza

del

sentimento

Cabanis, parce qu'il en est - on vient de le voir - le théoricien; Pinel, parce que, médecin à Bicêtre en 1793 puis à la Salpêtrière en 1795, il est le praticien par excellence des insensés atteints d'affections morales; Moreau de la Sarthe, parce que, chirurgien aux armées en 1793-1794, il a acquis l'expérience du praticien de terrain en charge de soldats aux troubles et pathologies multiples, surtout à Nantes entre les théâtres d'opérations militaires de Vendée et de Bretagne. Pour cette triade de médecins,

1/ fréquentant peu ou prou les mêmes institutions savantes médicales,

2/ attachée au néo-hippocratisme des Lumières ${ }^{91}$ sur lequel repose le naturalisme thérapeutique contempteur de la poly-pharmacie,

3/ adepte de la réunion de la philosophie, de l'histoire naturelle de l'homme et de la médecine dans une grande science de l'homme ${ }^{92}$,

Pour cette triade de médecins donc, le principe premier de l'unité de l'homme et le principe second de la réciprocité des rapports du physique et du moral - nous dirions du psychologique - ne peuvent que conduire à cette vérité que les passions sont à la fois objets et instruments du traitement médical ${ }^{93}$. Passions, contre-passions, telle est la vraie thérapeutique des affections complexes qui touchent l'être humain.

Dans de telles conditions, que penser de la bonne vieille morale de la maîtrise des passions? Tout simplement, qu'elle est devenue, qu'elle est hors sujet!

91. C'est d'ailleurs chez Hippocrate que se trouve déjà le jeu passions / contre-passions. N'est-ce pas cela en effet qui est à l'œuvre dans le récit de Démocrite d'Abdère, fou de rire par compensation de son affliction démesurée au spectacle de ses contemporains?

92. La Nosographie philosophique ou la méthode de l'analyse appliquée à la médecine de Pinel, monument de l'histoire de la science médicale, est aussi de l'an VI.

93. Ce qui va donner le sujet de thèse (dédiée à Pinel) de celui que la tradition considère comme le grand homme de la psychiatrie française, Esquirol: Des Passions, Considérées comme Causes, Symptômes et moyens curatifs de l'Aliénation mentale; Thèse Présentée et soutenue à l'Ecole de Médecine de Paris, le 7 Nivôse an 14 [...], soit 8 ans après notre an VI et sa mutation radicale! 
Parte seconda

Sentimento e retorica

aA 

Metamorfosi dei

Lumi 10.

L'emergenza del

sentimento

\section{Madame du Deffand:} dall'estetica alla retorica dell'emozione

Debora Sicco

La marchesa Marie de Vichy Chamrond, meglio nota come Madame du Deffand, ha rivestito nel XviII secolo un ruolo di primo piano, sia in qualità di animatrice del celebre $s a$ lon di Saint-Joseph sia in qualità di corrispondente di molti importanti protagonisti della scena culturale e politica del tempo, fra i quali si annoverano Voltaire, Montesquieu, Maupertuis, d'Alembert, Julie de Lespinasse, il conte Scheffer (ambasciatore di Svezia), Horace Walpole, la duchessa di Choiseul. A partire da queste lettere, che hanno indotto Sainte-Beuve ad accostare la sua prosa a quella di Voltaire e a definirla «un de nos classiques par la langue et par la pensée, et l'un de plus excellents» ${ }^{1}$, si tenterà di mettere in luce come l'atteggiamento della marchesa nei riguardi dell'emozione sia mutato nel corso del tempo, accompagnando e anticipando la metamorfosi subita da tale idea negli anni del tournant des Lumières.

1. C.A. de Sainte-Beuve, Lettres de la marquise du Deffand, in Causeries du lundi, Garnier, Paris 1857, vol. 1, p. 412. Per quanto riguarda l'accostamento a Voltaire a cui si è accennato: «Elle est, avec Voltaire, dans la prose, le classique le plus pur de cette époque, sans même en excepter aucun des grands écrivains» (ivi, p. 413). 
Metamorfosi dei Lumi 10. L'emergenza del sentimento

60
Madame du Deffand, infatti, ha manifestato per buona parte della propria vita una spiccata avversione nei confronti dell'emozione in tutte le sue sfaccettature, nonché di ciò che definisce sprezzantemente "romanzesco". Lo testimonia, oltre alla corrispondenza con il président Hénault, la sua reputazione di aridità, rimasta viva negli anni; ad esempio, «Stendhal ha una vera prevenzione verso Madame du Deffand che giudica colpevole di un'arrogante negazione del sentimento e corifea di quel bon ton francese che maschera l'impotenza» ${ }^{2}$. Non si può, tuttavia, ignorare che a un certo punto il suo modo di intendere l'emozione muta radicalmente, come emerge con nettezza dal confronto tra il già accennato scambio epistolare con Hénault e quello, molto più corposo, con Horace Walpole. Indubbiamente, la marchesa ha aderito a lungo al severo codice estetico-comportamentale tipico della mondanità settecentesca, rispettando con scrupolo le regole mondane e l' "etichetta" che contrassegna anche il genere epistolare. Tuttavia, da un certo momento in poi, ella passa da una concezione estetico-letteraria, che guarda con diffidenza se non con aperto disprezzo all'emozione, a una vera e propria retorica dell'emozione, fondata sul valore persuasivo di quest'ultima.

L'ipotesi alla base di questo articolo è che all'origine di questa "metamorfosi" vi sia la singolare convergenza tra una significativa evoluzione del clima culturale e un evento biografico. Per quanto riguarda la prima, la sua più emblematica testimonianza in Francia è la pubblicazione, nel 1761, della Nouvelle Héloïse di Rousseau; per quanto invece riguarda il secondo, si allude all'incontro con Horace Walpole, avenuto nel 1766, che costituisce una sorta di spartiacque tra due diversi modi di rapportarsi all'emozione e al sentimento da parte di Madame du Deffand. Lettrice attenta, ella sa apprezzare la nuova letteratura sentimentale, romanzi inglesi in primis, e si sofferma a riflettere sulla possibilità di una retorica del sentimento che non sconfini nel cattivo gusto. La centralità che lo stile $\mathrm{e}$, più in generale, il buon gusto letterario hanno fra $\mathrm{i}$ suoi valori rende particolarmente interessante l'intreccio fra emozione e retorica che emerge dalla sua corrispondenza,

2. B. Craveri, Madame du Deffand e il suo mondo, Adelphi, Milano 1982, pp. 108109; cfr. Stendhal, De l'amour, in Euvres complètes, a cura di V. Del Litto, 1963, vol. III, pp. 145-46. 
soprattutto da quella con Walpole. Qui, infatti, la marchesa si confronta in prima persona con la difficoltà di enucleare la nuova visione retorica della sensibilità che, pur convincendola intimamente, non riesce a soppiantare del tutto la precedente concezione estetico-letteraria, alla quale peraltro anche Walpole sembra ostinatamente fedele.

\section{2. «Je n’ai ni tempérament ni roman». La corrispondenza con}

\section{Hénault}

Nel corso della sua lunga vita (25 settembre 1696 - 23 settembre 1780), Madame du Deffand ha scelto di cimentarsi in un solo genere: quello epistolare. Questa esclusiva preferenza per una scrittura occasionale e dialogica, che la induce fra l'altro a respingere il consiglio di Voltaire di scrivere un diario, rispecchia sia la sua personalità sia la socialità tipica dei salotti. Per riprendere le parole di Benedetta Craveri, «questo genere di comunicazione estemporanea è profondamente congeniale alla marchesa la cui intelligenza, educata alla scuola mondana, ha sempre bisogno di un interlocutore per esprimersi, si nega ogni iniziativa e preferisce piuttosto reagire alle sollecitazioni degli altri» ${ }^{3}$. In un certo senso, la scrittura epistolare rappresenta la prosecuzione delle conversazioni del salon ${ }^{4}$ di Saint-Joseph, e come queste deve avere uno stile ironico e lieve, espressione di un esercizio dell'intelligenza che non sconfina mai nella pedanteria.

Madame du Deffand si conforma dunque, almeno inizialmente, a una precisa estetica epistolare, influenzata dal contesto del salon: assai raramente di carattere privato, le lettere erano invece destinate a essere lette ad alta voce e a intrattenere gli ospiti, che traevano spunto dagli aggiornamenti degli assenti per conversare e divertirsi. Non c'era spazio per una corrispondenza incentrata sulla comunicazione di stati d'animo personali e di sentimenti autentici, che sarebbe stata percepita come inopportuna. L'adesione della marchesa a questo modo di intendere la comunicazione epistolare è testimoniata dalle lettere che, durante il suo soggiorno a Forges per una cura termale nell'estate del 1742, ella ha scambiato con François

3. B. Craveri, Madame du Deffand e il suo mondo, cit., p. 147.

4. Di fatto, le lettere permettevano realmente a chi era lontano di continuare a partecipare alla vita del salon, ovvero all'essenza della vita mondana e culturale settecentesca, contribuendo a sua volta ad arricchirla. 
Metamorfosi dei Lumi 10. L'emergenza del sentimento

Hénault, a tutti noto come le président per la sua carica di magistrato presso il parlamento di Parigi ${ }^{5}$. Tali lettere rivelano sia il tono della loro lunga relazione, incentrata sulla comune vita sociale piuttosto che sulla profondità dei sentimenti, sia l'insofferenza e lo scetticismo di Madame du Deffand nei confronti del sentimento e delle sue manifestazioni.

Ella prende apertamente le distanze da ogni sentimentalismo persino quando si tratta di esprimere a Hénault il valore che attribuisce alla sua compagnia: «Vous m'êtes aussi nécessaire que ma propre existence, puisque, tous les jours, je préfère d'être avec vous à être avec tous les gens que je vois: ce n'est pas une douceur que je prétends vous dire, c'est une démonstration géométrique que je prétends vous donner» ${ }^{6}$. Rifuggendo imbarazzanti dichiarazioni e frasi melense, la marchesa riconduce i sentimenti all'ambito delle scienze esatte; l'ammissione di apprezzare più di ogni altra la compagnia dell'interlocutore si riduce così a una semplice constatazione di fatto. Nel complesso, la sua corrispondenza con Hénault, pur caratterizzata da un tono amabile, lascia trapelare un certo disincantato distacco fra loro e si concentra più sui fatti che sui sentimenti ${ }^{7}$ : ogni lettera rappresenta una sorta di resoconto degli eventi salienti delle loro giornate, piene di incontri, nomi propri e dettagli mondani.

Qualche decennio dopo, lettere simili saranno espressamente richieste da Walpole alla marchesa, assai recalcitrante ad assecondarlo; ma negli anni Quaranta del Settecento ella è ancora ben lontana dall'idea che si possano affidare le proprie emozioni a uno scambio epistolare. La testimonianza più emblematica di questo atteggiamento è senza dubbio la risposta alla lettera inviatale da Hénault il 12 luglio 1742. Al président, che pur conoscendone il carattere si arrischia a scriverle di aver particolarmente sentito la sua mancanza in una bella serata di chiaro di luna, ella replica senza alcuna accondiscendenza $^{8}$, concludendo seccamente: «Je n’ai ni tempérament ni

5. Hénault è stato anche membro dell'Académie française. Ha scritto opere teatrali e un Abrégé chronologique de l'histoire de France (1744).

6. Madame du Deffand, lettera a Hénault del 5 luglio 1742, in Lettres de Madame du Deffand (1742-1780), Impression-Novoprint, Barcelona 2018, p. 31.

7. B. Craveri, Madame du Deffand e il suo mondo, cit., p. 44: «La minimizzazione del sentimento sembra essere il filo conduttore di questo dialogo epistolare in cui la diffidenza prevale sulla confidenza, l'ironia sulla tenerezza».

8. «C'est le clair de lune, ce sont de certaines circonstances qui font que vous 
roman $»^{9}$. La critica che rivolge alla romantica galanteria di Hénault riguarda tanto il piano del sentimento quanto quello dello stile, ai suoi occhi costantemente intrecciati. In primo luogo, la dichiarazione di Hénault, suggerendo la dipendenza del sentimento - quantomeno della sua intensità - da mutevoli circostanze esteriori come il chiaro di luna, può essere letta come l'espressione maldestra di una carenza di sentimento, piuttosto che della sua sovrabbondanza. In secondo luogo, il président ha la non lieve colpa di aver fatto ricorso a un linguaggio degno di un eroe della letteratura preziosa, che Madame du Deffand detesta, e in cui ravvisa una forma di metafisica dell'emozione, lontana dall'emozione autentica.

Dal suo punto di vista, la forma ha una grande importanza: se inappropriata, essa può infatti compromettere anche il contenuto, inducendo a dubitare della sua autenticità o gettando su di esso il discredito del ridicolo. Da questa convinzione derivano serie riserve sull'opportunità di esprimere i propri sentimenti, soprattutto attraverso le formule abusate della scrittura epistolare o slanci poetici più o meno convenzionali. Madame du Deffand cercherà di insegnarlo, qualche anno più tardi, anche alla nipote Julie de Lespinasse: «Ou il ne faut point écrire à ses amis, ou bien il faut que les lettres soient une conversation; les assurances d'attachement et d'amitié sont si communes, et si fort d'usage pour ceux qui ne s'aiment point, que ceux qui s'aiment doivent s'en abstenir ${ }^{10}$. La raccomandazione alla nipote, così come il rimprovero rivolto a Hénault, è anche un invito a non cadere nel cattivo gusto, inteso come assenza di naturalezza ${ }^{11}$ e contrapposto dalla marchesa al "romanzesco", a cui si accompagnano inesorabilmente banalità e ridicolo.

me désirez; je suis regrettée et souhaitée suivant les dispositions où la beauté du temps met votre âme; moi, je vous désire partout, et ne sache aucune circonstance qui put me rendre votre présence moins agréable» (Madame du Deffand, lettera a Hénault del 14 luglio 1742, in Lettres, cit., p. 41).

\section{Ibidem.}

10. Madame du Deffand, lettera a Mlle de Lespinasse del 16 gennaio 1753; citazione tratta da B. Craveri, Madame du Deffand e il suo mondo, cit., p. 189. Cfr. anche Madame du Deffand, lettera a Walpole del 20 giugno 1779, in Lettres, cit., p. 895: «Je haïs plus que jamais les phrases et les lieux communs, ils dénotent une disette de sentiments et de pensées».

11. Cfr. Madame du Deffand, lettera a Walpole del 17 maggio 1767, in Lettres, cit., p. 183: «Le bon goût est ce qui approche de la nature, ou ce qui imite parfaitement ce qu'on veut représenter». 
Metamorfosi dei

Lumi 10.

L'emergenza

del

sentimento

Sulla base di queste premesse, è meglio astenersi dall'esprimere i propri sentimenti, lezione che Hénault è costretto a fare propria. Così, egli risponde alla marchesa con un'irresistibile, riuscita parodia del sentimentalismo, rinnegato con una carrellata dei suoi più comuni clichés:

Ce que vous appelez roman dans votre lettre, les souvenirs, le clair de lune, etc. il me semblait que tout cela n'était point ridicule. Eh bien, soit! Je vous demande pardon pour tous les ruisseaux passés, présents et à venir, pour leurs frères les oiseaux, pour leurs cousins les ormeaux et pour leurs bisaieuls les sentiments. M'en voilà corrigé, et mes lettres ne seront plus qu'agréables pour vous, par tout ce que je pourrais ramasser des nouvelles de la ville et que j'imaginerai qui pourra vous amuser. Je reprends donc le style historique, et je ne parlerai de moi que quand cela amènera des faits ${ }^{12}$.

In questo passo, Hénault, pur mettendo inizialmente in dubbio l'esistenza di una connessione necessaria tra ciò che Madame du Deffand definisce "romanzo" e il ridicolo, promette di rinunciare a ogni forma di sentimentalismo e di assecondare in futuro i suoi desideri, limitandosi nelle sue lettere alla cronaca delle novità parigine che potrebbero interessarla. Una trentina di anni dopo, la marchesa si troverà in un'analoga posizione con Walpole, ma con una grande differenza. Infatti, mentre la corrispondenza con Hénault rappresenta un ottimo esempio di una concezione più arcaica e prettamente estetica dell'emozione, dominata dalla preoccupazione dell'efficacia letteraria, nelle lettere a Walpole Madame du Deffand tenterà almeno di abbozzare una retorica dell'emozione, capace di dare spazio all'espressione di sentimenti autentici, non artefatti e simulati come quelli delle formule epistolari o della letteratura preziosa.

3. «Soyons amis, mais sans amitié... Aimons-nous sans nous aimer»: la corrispondenza con Walpole

Fra le ragioni del radicale mutamento d'opinione di Madame du Deffand sulla possibilità di esprimere l'emozione, vi è indubbiamente l'incontro avvenuto nel 1766 con Horace

12. C.-J.-F. Hénault, lettera a Madame du Deffand del 18 luglio 1742, in Correspondance complète de la Marquise du Deffand avec ses amis le président Hénault, Montesquieu, d'Alembert, Voltaire, Horace Walpole (1865), Slatkine, Genève 1989, 2 voll., vol. I, p. 69. 
Walpole, diventato poi celebre come autore del romanzo gotico The Castle of Otranto. Questo inglese, di vent'anni più giovane, ispira presto alla quasi settantenne ${ }^{13}$ marchesa, cieca da circa dodici anni, un sentimento più simile all'amore che all'amicizia, ben definito da Lionel Duisit come «amitié passionnée» ${ }^{14}$. Sulla base di tali premesse, non sorprende che ella volesse affidare alla corrispondenza intrapresa al momento del ritorno in Inghilterra di Walpole sia i propri sentimenti di amicizia/amore nei suoi confronti sia le proprie più intime riflessioni, svelandogli così la sofferenza di un'esistenza tiranneggiata senza tregua dalla solitudine e dalla noia, malgrado le apparenze mondane. Walpole, al contrario $^{15}$, attraverso queste lettere aspirava a delineare un affresco della società del suo tempo e a ritrovare lo spirito del secolo di Luigi XIV.

Egli temeva terribilmente il ridicolo ${ }^{16}$, ma le sue frequenti schermaglie epistolari con la marchesa sono principalmente ascrivibili alla loro diversa visione della sensibilità e dell'emozione. Mentre Walpole continua a percepire ogni manifestazione emotiva come elemento perturbatore dell'estetica epistolare, Madame du Deffand, incoraggiata dalla tardiva esperienza biografica del sentimento, è sempre più convinta dalla nuova visione retorica dell'emozione. Se già all'epoca delle lettere a Hénault ella percepiva i limiti di un discorso puramente estetico, come dimostra la sua ferma avversione per le effusioni epistolari fini a se stesse, è soltanto nella corrispondenza successiva che propone un'alternativa, provando ad argomentare a favore della nuova concezione retorica

13. Cfr. a questo proposito C. Ferval, Madame du Deffand. L'Esprit et l'amour au XVIII siècle, Fayard, Paris 1933, p. 8: «C'est en plein hiver de l'âge que, pour la première fois, fortement, profondément, sa sensibilité sera émue».

14. L. Duisit, Madame du Deffand épistolière, Droz, Genève 1963, p. 11.

15. Cfr. a questo proposito anche B. Craveri, Madame du Deffand e il suo mondo, cit., pp. 373-374: «Walpole e la marchesa ripongono nell'attività epistolare aspettative molto diverse».

16. Madame du Deffand, che la viva simpatia provata da subito nei suoi confronti non ha privato della caratteristica lucidità, ne è consapevole: «Un des principaux traits du caractère de Walpole, et l'on peut dire (parce qu'il le portait à l'excès), une de ses principales faiblesses, était la crainte de se rendre ridicule». Préface, in Lettres de la marquise Du Deffand à Horace Walpole, depuis compte d'Oxford, écrites dans les années 1766 à 1780; auxquelles sont jointes des lettres de Madame du Deffand à Voltaire, écrites dans les années 1759 à 1775, publiées d'après les originaux déposés à StarwberryHill, Treuttel et Würtz, Paris-Strasbourg 1812, 4 voll., vol. I, p. IV. 
Metamorfosi dei

Lumi 10.

L'emergenza

del

sentimento dell'emozione, principalmente attraverso i romanzi inglesi. Nell'estate del 1742, ella conosce già Pamela di Richardson, di cui vorrebbe non aver ancora concluso la lettura; il peculiare rapporto con Walpole sembra aver però affinato in lei la sensibilità per quel genere letterario, con tutte le sue implicazioni. Ciò spiega forse anche le sue reazioni ambivalenti alla Nouvelle Héloïse di Rousseau, opera che ha rivestito un ruolo preponderante nell'affermazione del clima culturale evocato all'inizio.

Infatti, pur evocando spregiativamente in più di una lettera la Nouvelle Héloïse, Madame du Deffand trova che in questo libro vi siano «des endroits fort bons» ${ }^{17}$. Scrive inoltre di essere impegnata a rileggerlo, e questo lascia supporre un certo interesse da parte di una donna che non si fa scrupolo ad abbandonare i libri che le dispiacciono e che dichiara apertamente di ignorare molti dei volumi da lei posseduti a favore di un numero relativamente ristretto di letture. Tuttavia, benché nel giudicare l'autore non si faccia fuorviare dalla propria antipatia personale nei suoi confronti ${ }^{18}$, Madame du Deffand non può che restare perplessa di fronte allo stile di Rousseau. Il vigore con cui riesce a rendere e a trasmettere le emozioni la attrae profondamente, ma ella resta sempre un'aristocratica del secolo di Luigi XIV, che preferisce la nobile misura agli eccessi dettati dalla passione e non esita a farsi portavoce di una crociata in difesa del classicismo. Ella ammette di trovare più congeniale lo stile di Voltaire, paragonando la chiarezza di Rousseau a quella dei lampi e il suo ardore a quello della febbre. In altri termini, pur subendo suo malgrado il fascino di Rousseau e riconoscendone confusamente la grandezza, Madame du Deffand non lo sente proprio al punto da rinnegare in suo nome il classicismo della propria formazione.

Queste oscillazioni emergono con nettezza nella corrispondenza con Walpole, tanto più che egli la invita ripetutamente a non cadere nel cattivo gusto delle esternazioni sen-

17. Madame du Deffand, lettera a Walpole del 26 giugno 1768, in Lettres cit., p. 232.

18. Cfr. ad esempio la lettera del 25 giugno 1764. Nell'undicesimo libro delle Confessions scopriamo che quest'antipatia era condivisa, nonostante un iniziale slancio simpatetico suscitato in Rousseau dalla cecità della donna. J.-J. Rousseau, Confessions, in Euvres complètes, a cura di B. Gagnebin e M. Raymond, Gallimard, Paris 1959-1995, 5 voll., vol. I, p. 555. 
timentali, ovvero a eliminare ogni dichiarazione di amicizia e ogni discorso triste, a evitare il piano personale e a trasformare le sue lettere in una sorta di gazzetta ${ }^{19}$ : «Si vous voulez que je vous lise, racontez-moi des histoires, des commérages mondains avec beaucoup de noms propres et ne me parlez jamais de vos sentiments» ${ }^{20}$. Queste parole rivelano chiaramente sia il carattere imperioso di Walpole, che non si fa scrupoli a essere brusco ai limiti della scortesia, sia la sua insofferenza a ogni effusione sentimentale. La sua brutale sincerità si accompagna a una sorta di ricatto: se Madame du Deffand non asseconderà le sue aspettative, egli non leggerà più le sue lettere. La marchesa, avendo a cuore la prosecuzione della corrispondenza, talvolta tenta di rabbonirlo conformandosi ai suoi desideri, talaltra tenta di aggirare il suo veto di parlare di sentimenti, persuasa che «on n'est point maître de ses sensations ${ }^{21}$. Questa considerazione, in virtù della quale le secche esortazioni di Walpole a correggersi suonano tanto irrealizzabili quanto tiranniche, riguarda sia i sentimenti che ella nutre nei suoi confronti sia la tristezza che egli le rimprovera. Anche la tristezza, infatti, è ascrivibile in parte al temperamento, in parte alle cattive condizioni di salute di Madame du Deffand, che in entrambi i casi può solo subire l'influenza della componente fisica su quella morale e constatare l'impotenza della volontà.

Di particolare interesse, nel corso di questa corrispondenza durata quasi quindici anni ${ }^{22}$, sono i tentativi della marchesa di respingere le critiche di Walpole e di rivendicare il proprio diritto a esprimere ciò che sente, senza censure

19. A questo proposito, cfr. M. Charrier-Vozel, Madame du Deffand épistolière: de la lettre à la gazette, «Revue de l'aire»,38 (2012), pp. 133-144.

20. Citazione tratta da A. Maurois, Morceaux choisis, a cura di E.G. Le Grand, Cambridge University Press, Cambridge 1931, p. 68. Il capitolo VII di quest'opera è interamente dedicato a Madame du Deffand.

21. Madame du Deffand, lettera del 20 marzo 1772, in Lettres, cit., p. 493. Madame du Deffand aveva affermato qualcosa di analogo già nella lettera del 15 luglio 1770: «Je laisse toujours les portes de mon âme ouvertes pour y recevoir le plaisir; je désirerais de barricader celle par où entre le regret, l'ennui et la tristesse; mais mon âme est une chambre dont le destin ou le sort ne m'ont pas donné la clef» (ivi, p. 362).

22. Nel corso di questa corrispondenza, i due si scambiano circa 1700 lettere, 955 delle quali sono giunte fino a noi. Ben 840 di queste sono di Madame du Deffand, soltanto 100 di Walpole (e per la maggior parte si tratta di frammenti), che aveva chiesto che le altre fossero distrutte. Cfr. B. Craveri, Madame du Deffand e il suo mondo, cit., p. 379 . 
Metamorfosi

dei

Lumi 10.

L'emergenza

del

sentimento

imposte. Tale rivendicazione affonda le sue radici in un'esigenza di sincerità: «Vous voyez à quel point je suis triste; ne m'en sachez pas mauvais gré, et donnez-moi la liberté de me montrer à vous telle que je suis» ${ }^{23}$. Per non dover continuamente dissimulare i propri sentimenti, Madame du Deffand propone che ognuno di loro possa esprimersi come preferisce, senza per questo andare incontro a umilianti rimproveri: «Croyez-vous me faire plaisir en me répétant sans cesse que vous ne voulez point d'amis, que vous ne voulez point de liaisons? Cela est dur et triste à entendre. Supportez mes douceurs comme je supporte vos rigueurs; parlons chacun notre langue et vivons en paix» ${ }^{24}$. Purtroppo, però, Walpole è inflessibile nel rifiutare una corrispondenza basata sul tono dell'amicizia e della confidenza, costringendola spesso a ritornare sui suoi passi e a fare ammenda per quanto ha osato scrivere.

Così, al tentativo di rivendicare, quando e per quanto possibile, la legittimità di esprimere l'emozione nel più ampio senso del termine, Madame du Deffand alterna diverse e brillanti strategie retoriche. Un esempio particolarmente riuscito è offerto dalla lettera del 30 aprile 1768, nella quale «elle parodie le style allégorique des héros de l'Astrée, improvisant une sorte de réplique alphabétique de la carte du tendre» ${ }^{25}$ :

J'ai déchiré mon dictionnaire à la lettre A, amitié, affection, ardeur, attendrissement. Pour amour, affectation et artifice, ils n'y ont jamais été. J'y laisserai, si vous le permettez, attention [...]. Au C, caresse, contrainte seront retranchés; constance et confiance resteront. Jugez du reste de l'alphabet par ce commencement, qu'avez-vous à craindre de mes lettres à l'avenir? ${ }^{26}$

In più di un'occasione ella si spinge fino a parodiare lo stesso Walpole; per esempio, nella lettera del 30 settembre 1766 gli fa elencare i capi d'accusa che senza dubbio vorrebbe presentare contro di lei, mostrandone la leggerezza: come potrebbe l'innocente richiesta di ricevere due volte alla settimana noti-

23. Madame du Deffand, lettera a Walpole del 1 novembre 1766, in Lettres, cit., p. 161

24. Madame du Deffand, lettera a Walpole del 15 giugno 1767, citazione tratta da

B. Craveri, Madame du Deffand e il suo mondo, cit., p. 413.

25. L. Duisit, Madame du Deffand épistolière, cit., pp. 61-62.

26. Citazione tratta da A. Maurois, Morceaux choisis, cit., p. 72. 
zie di un amico malato fare di lui un ridicolo personaggio di romanzo? In questo modo, ella cerca di richiamarlo al buon senso, talvolta anche ostentando e ingigantendo le "intemperanze" che Walpole le rimprovera, sia per alleggerire con tono scherzoso la tensione che costantemente attraversa il loro scambio epistolare, sia per mettere in luce ogni possibile attenuante in proprio favore. Dopotutto, ella è persuasa che ognuno è fatto a modo proprio, e accetta anche Walpole per quello che è, insieme persino all'apparente assurdità di essere amici «sans amitié ${ }^{27}$.

4. «Le ton de roman est à la passion ce que le cuivre est à l'or»: una retorica dell'emozione

Il difficoltoso tentativo di conciliare la volontà di esprimere ciò che prova con le diverse aspettative di Walpole rende cruciale la riflessione di Madame du Deffand sulla retorica dei sentimenti. Innanzitutto, anche se il confine tra amicizia e amore non è così marcato e invalicabile come pretende che $\operatorname{sia}^{28}$, nelle arringhe a propria difesa ella tende a spostare il capo di imputazione dai sentimenti al linguaggio con cui questi vengono espressi. Il problema, a suo avviso, non è esprimere sentimenti ed emozioni, ma farlo convenientemente: l'errore non sta in quel che scrive, ma in come lo scrive. Di conseguenza, mentre lo stile è frequentemente messo sotto accusa e ritrattato, l'amicizia è ribadita persino nelle lettere scritte per ottenere il perdono dopo un'analoga confessione. Si può ipotizzare, come Benedetta Craveri, che nel far questo «Madame du Deffand è fondamentalmente sincera e probabilmente inconsapevole di eludere una realtà sentimentale con una categoria estetica» ${ }^{29}$. In ogni caso, la rivalutazione dell'emozione, intesa nel più ampio senso del termine, influisce anche sui giudizi estetici della marchesa, inducendola ad ampliare i propri orizzonti e a convincere altri della necessità di fare altrettanto.

27. Madame du Deffand, lettera a Walpole del 5 agosto 1766 . Citazione tratta da W. Klerks, Madame du Deffand. Essai sur l'ennui, Universitaire Pers, Leiden 1961, p. 44.

28. Cfr., ad esempio, la lettera del primo ottobre 1766. Madame du Deffand, Lettres, cit., p. 151.

29. B. Craveri, Madame du Deffand e il suo mondo, cit., pp. 385-386. 
Metamorfosi dei

Lumi 10.

L'emergenza

del

sentimento

Lettrice esigente, ella coglie con chiarezza i limiti di molte opere che, pur fedeli ai canoni letterari, non riescono a trasmettere alcuna emozione, preferendone altre malgrado la sua strenua difesa del classicismo in ambito letterario ${ }^{30}$. In compenso, apprezza le opere attraverso le quali le emozioni sono mirabilmente dipinte e trasmesse al lettore: come scrive in una lettera del primo marzo 1769, «j'aime qu'on me peigne les passions ${ }^{31}$. I suoi giudizi, spesso sorprendentemente attuali, si basano per sua esplicita ammissione su un criterio soggettivo: «Je ne juge que par sentiment; si je demande à mon esprit une opération quelconque, je reconnais alors que je n'en ai point du tout» ${ }^{32}$. Madame du Deffand ribadisce questa assoluta soggettività (e indipendenza) di giudizio in una lettera dell'8 agosto 1773: «Vous avez grand tort de me consulter; vous ne savez donc pas comment je juge? Par deux sensations, ennui ou plaisir; jamais je n'examine les causes ${ }^{33}$.

Proprio in questa lettera, in risposta alle osservazioni critiche di Walpole sul teatro francese (a suo avviso frutto dell'educazione più che di un'effettiva conoscenza delle passioni $^{34}$ ), Madame du Deffand sorvola piuttosto rapidamente su Shakespeare, che ancora non la convince appieno ${ }^{35}$, per esprimersi più diffusamente e positivamente sui romanzi inglesi ${ }^{36}$. Questi (ad esempio Tom Jones di Henri Fielding) le

30. Lionel Duisit ha messo bene in evidenza questa contraddizione: «D'un côté la marquise se sert du goût pour juger les ouvrages, mais en même temps, elle condamne les produits de ce goût en reconnaissant qu'ils leur manque quelque chose d'essentiel pour que l'âme en soit ébranlée» (Madame du Deffand épistolière, cit., p. 46).

31. Madame du Deffand, lettera a Voltaire del primo marzo 1769, in Lettres, cit., p. 274.

32. Madame du Deffand, lettera a Walpole del 28 giugno 1768, in Lettres, cit., p. 234.

33. Madame du Deffand, lettera a Walpole dell' 8 agosto 1773, in Lettres, cit., p. 579. Benché tale criterio non sia né pretenda di essere "scientifico", occorre riconoscere che «elle ne se trompe pas lorsqu'il s'agit de reconnaître le génie, même s'il choque ses goûts ou ses idées» (L. Duisit, Madame du Deffand épistolière, cit., p. 49).

34. Walpole si sofferma, in particolare, su Zaïre di Voltaire e Mithridate di Racine. 35. A proposito della ricezione settecentesca di Shakespeare, in cui in Francia ha rivestito un ruolo fondamentale Voltaire, cfr. K.E. Larson e H. Schelle (a cura di), The Reception of Shakespeare in Eighteenth-century in France and Germany, «Michigan Germanic Studies», 15 (1989); F. Ritchie e P. Sabor (a cura di), Shakespeare in the Eighteenth Century, Cambridge University Press, Cambridge 2012.

36. Cfr. a questo proposito G. Rageot, Madame du Deffand, Albin Michel, Paris 1937, p. 233: «Elle fut l'une des premières à goûter les romans anglais, dont elle vantait le naturel». 
sembrano superiori a quelli francesi, nonostante alcuni difetti fra cui spicca la lunghezza eccessiva: «À l'égard de vos romans, j'y trouve des longueurs, des choses dégoûtantes, mais une vérité dans les caractères (quoiqu'il y en a une variété infinie) qui me fait démêler dans moi-même mille nuances que je n'y connaissais pas» ${ }^{37}$. Convinta di questi pregi, Madame du Deffand non esita a illustrarli a Walpole, assai meno entusiasta dei romanzi inglesi, e perora la loro causa presso Voltaire, cercando di persuaderlo a leggerli:

Monsieur, vous n'avez point lu les romans anglais; vous ne les mépriseriez pas, si vous les connaissiez. Ils sont trop longs, je l'avoue, et vous faites un meilleur emploi du temps. La morale y est en action, et n'a jamais été traitée d'une manière plus intéressante. On meurt d'envie d'être parfait avec cette lecture, et l'on croit que rien n'est si aisés8.

È evidente in questo passo l'eco delle osservazioni di Diderot $^{39}$, il cui Éloge de Richardson è inviato dalla marchesa a Walpole; agli apoftegmi dei moralisti, ella preferisce nettamente la morale messa in atto dai personaggi, più coinvolgente $\mathrm{e}$ convincente. Nei romanzi inglesi, i personaggi (per esempio quelli di Richardson: Pamela, Clarissa, Grandisson) e i loro sentimenti sono dipinti secondo natura, quindi molto più efficacemente rispetto agli eroi di Calprènede ${ }^{40} \mathrm{o}$ di Mademoiselle de Scudéry. A suo avviso, a fare la differenza è appunto la capacità di toccare la sensibilità dei lettori, capacità che non esita a riconoscere anche nelle opere di alcuni autori francesi, ad esempio Sedaine: «Ce Sedaine a un genre qui fait un grand effet, il a trouvé de nouvelles cordes pour exciter la sensibilité; il va droit au cour, et laisse là tous les détours d'u-

37. Madame du Deffand, lettera dell' 8 agosto 1773, cit., p. 580.

38. Madame du Deffand, lettera a Voltaire del primo ottobre 1759, in Lettres, cit., p. 72.

39. Cfr. L. Duisit, Madame du Deffand épistolière, cit., p. 42, e, per la posizione di Diderot, L. Hunt, Inventing Human Rights: A History, W.W. Norton \& Co., New York, 2007, pp. 54-55; trad. it. La forza dell'empatia. Una storia dei diritti dell'uomo, Laterza, Roma-Bari 2010, p. 37: «Uno dei più eloquenti difensori del romanzo [Pamela] fu Diderot, autore dell'articolo sul diritto naturale dell'Encyclopédie ed egli stesso romanziere. Quando Richardson morì, nel 1761, Diderot scrisse un elogio nel quale paragonò lo scrittore ai maggiori autori dell'antichità: Mosè, Omero, Euripide e Sofocle».

40. Autore del romanzo Cassandre e di Cléopatre, opere a proposito delle quali Madame du Deffand scrive: «C'est le plus détestable style». Lettera del 3 dicembre 1779, in Lettres, cit., p. 909. 
Metamorfosi dei

Lumi 10.

L'emergenza

del

sentimento

ne métaphysique que je trouve détestable en tous genres» ${ }^{41}$. L'avversione per la metafisica professata senza mezzi termini dalla marchesa è, insieme a quella per il "romanzesco" evocata sopra, una costante del suo pensiero; lei stessa, nella lettera del 27 gennaio 1771 rivela: «C'est une antipathie naturelle que j'ai pour les croisades, et cela dès mon enfance. Je haïs Don Quichotte et les histoires de fous; je n'aime point les romans de chevalerie, ni ceux qui sont métaphysiques; j'aime les histoires et les romans qui me peignent les passions et les vertus dans leur naturel et leur vérité» ${ }^{42}$.

Questa totale mancanza di inclinazione per il "romanzesco" diventa per Madame du Deffand un vero e proprio tratto distintivo, al quale lei stessa fa appello in occasione della prima, inattesa e avvilente schermaglia epistolare con Walpole. Costui, di fronte a una confidenza che reputa eccessiva, esprime la propria insofferenza per le lagnanze dell'interlocutrice e ne critica pesantemente lo stile: «Suis-je fait pour être le héros d'un roman épistolaire? Et comment est-il possible, Madame, qu'avec autant d'esprit que vous avez, vous donnez dans un style qui révolte votre Pilade, car vous ne voulez pas que je me prenne pour un Orondate?» ${ }^{43}$. Questa accusa suscita l'incredula indignazione della marchesa, che ribatte: «Revenons aux emportements romanesques: moi, l'ennemie déclarée de tout ce qui en a le moindre trait, moi qui leur ai toujours déclaré la guerre, moi qui me suis fait des ennemis de tous ceux qui donnaient dans ce ridicule, c'est moi qui en suis accusée aujourd'hui!» ${ }^{44}$. Allorché Walpole ripeterà il rimprovero, ella tornerà a suggerire che sia lui a fraintenderne l'atteggiamento, adducendo a conferma di ciò la sua diversa reputazione: «Le reproche que vous me faites d'aimer le romanesque fait rire tous ceux qui me connaissent; jamais personne n'en a été moins soupçonné; je trouve assez singulier d'être si peu connue de vous» ${ }^{45}$.

Effettivamente, Madame du Deffand accomuna in un'uni-

41. Madame du Deffand, lettera a Voltaire del 16 luglio 1768.

42. Le Lettres portugaises, pubblicate anonime nel 1669 come traduzione di cinque lettere di una religiosa portoghese a un ufficiale francese, sono oggi ritenute dalla maggioranza degli studiosi un romanzo epistolare di Gabriel de Guilleragues.

43. Orondate è un personaggio del romanzo Cyrus di Madeleine Scudéry.

44. Madame du Deffand, lettera a Walpole del 21 aprile 1766, in Lettres, cit., p. 122.

45. Madame du Deffand, lettera a Walpole dell' 8 luglio 1772, in Lettres, cit., p. 528. 
ca condanna le elegie di Madame de la Suze, le Lettres portugaises $^{46}$ e i romanzi di Mademoiselle de Scudéry. Ella è anche molto critica nei riguardi della cognata di lei, colpevole a suo avviso di elemosinare l'amicizia di Bussy-Rabutin e di avere uno stile monotono e noioso. Ancora una volta, per Madame du Deffand la difficoltà consiste nell'esprimere in modo adeguato i propri sentimenti: quando è sincera, l'emozione non si traduce facilmente in parole, anzi, come Rousseau ha mostrato può arrivare a soffocare la parola. Tuttavia, a suo parere è comunque possibile esprimere l'emozione in una lettera: Madame de Sévigné lo dimostra. Quest'ultima rappresenta un fondamentale modello di scrittura epistolare sia per Madame du Deffand sia per Walpole ${ }^{47}$, che aveva nei suoi confronti un'autentica venerazione, al punto da definirla la sainte de Livry e da collezionarne autografi e altre "reliquie". Conscia di ciò, la marchesa tenta di servirsene in propria difesa, per convincere l'irascibile interlocutore che uno stile gradevole può far risultare anche il linguaggio dei sentimenti semplice e naturale.

Questa strategia è destinata a rivelarsi fallimentare: nonostante la sincera ammirazione per Madame de Sévigné, Walpole ritiene che dal suo epistolario andrebbero espunte le lettere in cui ella esprime nel modo più toccante il proprio amore di madre ${ }^{48}$, riducendola pressappoco a una redattrice di gazzette (lo stesso ruolo in cui avrebbe voluto confinare Madame du Deffand). Se la tenerezza è un errore da biasimare anche nelle lettere di colei che considera il modello dell'eloquenza epistolare, allora decisamente nessuno stile può renderla accettabile. Malgrado ciò, Madame du Deffand persevera nel tracciare una distinzione estetica fra diverse manifestazioni sentimentali e passionali. Mentre il "romanzesco" non cessa di essere sdegnosamente respinto, la passione

46. Ivi, pp. 136-137 (lettera del 26 maggio 1766): «Vous m'avez irritée, troublée, et qui pis est, gelée: me comparer à Madame de la Suze! Me menacer de m'écrire pour réponse une portugaise! Ce sont les deux choses du monde que je hais le plus; l'une pour sa dégoûtante et monotone fadeur, et l'autre pour ses emportements indécents».

47. Cfr. in merito J.-Y. Huet, Madame de Sévigné en Angleterre: Horace Walpole et Madame du Deffand, «Revue de l'histoire littéraire de la France», 96 (1996), n. 3, pp. 404435 .

48. A proposito di queste lettere alla figlia, cfr. A. Bernet, Madame de Sévigné. Mère passion, Perrin, Paris 1996. 
Metamorfosi

dei

Lumi 10.

L'emergenza

del

sentimento

ha diritto a uno spazio, purché sincera ed esposta con grazia, ossia a patto di rispettare l'irrinunciabile connubio di naturalezza e stile che la marchesa identifica con il buon gusto. Ai suoi occhi, questo ideale si realizza compiutamente nelle lettere di Madame de Sévigné:

Tout son esprit n'était que passion, imagination et sentiment; elle ne voyait rien avec indifférence et peignait les amours de sa jardinière avec la même chaleur qu'elle aurait peint ceux de Cléopatre et de madame de Clèves. Ce n'est point qu'elle fût romanesque, elle en était bien loin; le ton du roman est à la passion ce que le cuivre est à l'or ${ }^{49}$.

Per quanto insista su questa distinzione, di fatto piuttosto sfuggente e soggettiva, Madame du Deffand si ritrova imprigionata nell' impasse del sentimento, che non può più limitarsi a ignorare, ma di cui non riesce nemmeno a rendere conto in maniera soddisfacente. Il riconoscimento dei limiti del classicismo, avvertiti con sempre maggior forza, non la conduce al suo completo superamento. Impantanata in questa dolorosa contraddizione, nell'ultimo periodo della sua vita Madame du Deffand oscilla fra l'inclinazione a un aperto riconoscimento del valore cruciale del sentimento e il rifiuto di ammetterlo come valida fonte della creazione artistica (da cui deriva l'ostentato fastidio tanto verso la nuova quanto verso l'antica Eloisa ${ }^{50}$ ). La sua corrispondenza, in particolare quella con Walpole, è emblematica dell'affermarsi nel Settecento di una mutata sensibilità, che conduce allo scontro tra una visione prevalentemente estetica dell'emozione e una nuova visione retorica della stessa. Lungi dal rimanere estranea o indifferente a questo radicale cambiamento, Madame du Deffand ne fa l'esperienza in prima persona, finendo però per rimanere drammaticamente divisa fra l'esigenza di esprimere l'emozione e la difficoltà di riuscirvi adeguatamente.

49. Madame du Deffand, lettera del primo febbraio 1772.

50. Cfr. ad esempio Madame du Deffand, lettera a Walpole del 21 aprile 1766, in Lettres, cit., pp. 122-123: «Soyez Abailard, si vous voulez, mais ne comptez pas que je suis jamais Héloïse. Est-ce que je ne vous ai jamais dit l'antipathie que j'ai pour ces lettres-là? J'ai été persécutée de toutes les traductions qu'on en a faites et qu'on me forçait d'entendre; ce mélange, ou plutôt ce galimatias de dévotion, de métaphysique, de physique, me paraissait faux, exagéré, dégoûtant». 
Metamorfosi dei

Lumi 10.

L'emergenza del

sentimento
I linguaggi delle emozioni in Inghilterra tra Sette e Ottocento: arte, poesia e teatro

Claudia Corti

Vorrei iniziare la mia argomentazione muovendo sia dal ruolo istituzionale e normativo svolto dalla Royal Academy (supremo vanto della cultura britannica), sia dalla sempre più esuberante critica giornalistica, a proposito del dibattito sui rapporti tra letteratura e arti figurative, che sta alla base del fenomeno epocale della primaria importanza attribuita a emozioni, passioni, sensazioni e sentimenti, in terra d'Albione, a partire dal primo Settecento fino almeno alla seconda metà dell'Ottocento. Il principale tratto distintivo di tale fenomeno culturale è l'assorbimento, nel tradizionale parallelo dell' ut pictura poèsis, dell'arte drammatica, quale punto di intersezione tra prerogative e funzioni delle famose «arti sorelle» ${ }^{1}$.

Alla Royal Academy, sono soprattutto le lezioni di Henry Fuseli (anglicizzazione del nome dell'artista svizzero Hein-

1. Si veda C. Corti e M.G. Messina (a cura di), Poesia come pittura nel Romanticismo inglese, Liguori, Napoli 2004. Inoltre, studi fondamentali in tale prospettiva sono J. Barrell, The Political Theory of Painting from Reynolds to Hazlitt, Yale University Press, New Haven-London 1986; S.S. Kenny (a cura di), British Theatre and the Other Arts 1660-1800, Folger Library, Washington 1990. 
Metamorfosi dei

Lumi 10.

L'emergenza

del

sentimento rich Füssli, trasmigrato in Inghilterra) e di John Opie (inglese nativo), a costituire i principali referenti concettuali.

Fuseli, che comincia a insegnare nel 1801, recupera subito, fin dall'esordio accademico, «the brilliant antithesis ascribed to Simonides, that painting is mute poesy, and poetry speaking painting», distinguendo accuratamente, nella loro comunità funzionale, tra le rispettive modalità espressive $\mathrm{e}$ comunicative:

As poetry and painting resemble each other in their uniform address to the senses, for the impression they mean to make on our fancy, and by that on our mind, so they differ as essentially in their materials and their modes of application, which are regulated by the diversity of the organs they address - ear and eye. Successive action communicated by sounds, and time, are the medium of poetry; form displayed in space, and momentaneous energy, are the elements of painting ${ }^{2}$.

Pare semplice intuire come simili affermazioni abbiano costituito il terreno dell'elevazione dell'arte drammatica a speciale funzione di intercomunicabilità tra parole e figure, intermediazione di tempo e spazio, mutuazione di azione e forma.

Sembra evidente come il teatro (tanto amato dal pittore svizzero) divenga, su tali basi teoriche, una perfetta combinazione delle arti sorelle, nella misura in cui condivide parole per l'orecchio e immagini per l'occhio; l'azione del poema e la forma del quadro; la dimensione cronologica del racconto e la dimensione spaziale della tela. E sembra altrettanto pleonastico aggiungere come quell' «energia», richiesta al dipinto in forma solo «momentanea», fissata nel momento della pennellata, abbia invece un vasto orizzonte di esplicazione nella durata del testo drammatico.

Tuttavia, laddove emerge in maniera definitiva la superiorità assoluta dell'arte drammatica, è nella inimitabile sua capacità di rappresentare le emozioni; laddove sia il poeta sia l'artista sono limitati dall'artificiosità e l'astrazione che distinguono i generi di appartenenza, il drammaturgo possiede l'esclusiva risorsa di affidare al corpo dinamico dell'attore, ai suoi sensi e alle sue prerogative fisiche, il compito di raffigu- 
I linguaggi

delle emozioni

in Inghilterra

tra Sette

e Ottocento:

arte, poesia e teatro

rare in parole, gesti e multiformi posture, le infinite e mobili sfumature del carattere di un personaggio; in tal modo sollecitando, per effetto riflessivo, le più forti reazioni emotive nel pubblico:

The exhibition of character in the conflict of passions is the legitimate sphere of dramatic invention. It inspires, it agitates us by reflective self-love, with pity, terror, hope, fear; whatever makes events, and time and place, the minsters of character and pathos, let fiction of reality compose the tissue, is its legitimate claim: it distinguishes and raises itself above historic representation by laying the chief interest on the actors, and moulding the fact into mere situations contrived for their exhibition ${ }^{3}$.

A Fuseli fa eco Opie, che insegna all'Accademia nel 1806, riproponendo il parallelo poesia/pittura con implicazioni trasparentemente drammaturgiche:

To a painter or poet nothing can be useless: whatever is great, whatever is beautiful, whatever is interesting, and whatever is dreadful, must be familiar to his imagination, and concur to store his mind with an inexhaustible variety of ideas $[. .$.$] to embellish sentiment and give effect of truth.$ [...] He must, further, observe the power of the passions in all their combinations, and trace their changes ${ }^{4}$.

La correlazione del bello al sinergismo tra funzioni mentali e corporee, nonché la capacità del letterato o artista di rappresentare le passioni, appaiono decisamente investite di intenzioni metadrammatiche.

Non la pensavano diversamente i critici teatrali del periodo $^{5}$. Le riviste più attive nel dibattito di idee attorno alla capacità delle arti sorelle di copiare le emozioni sono sicuramente, in Inghilterra, «The Artist» e «The Examiner», dove si assiste alla progressiva emancipazione dell'arte drammatica dal ruolo di puro intrattenimento a quello, più profondo, di disciplina estetica e morale, di valenza non inferiore a quella delle sue consorelle. Due dati emergono infatti: che il teatro

3. Ivi, pp. 195-196. Corsivi dell'autore.

4. J. Opie, Lecture II, On Invention, in R.N. Wornum (a cura di), Lectures on Painting by the Royal Academicians, Barry, Opie, and Fuseli, Bohn, London 1848, p. 270.

5. Per un'ottima sintesi dell'argomento, si veda soprattutto J.R. Heller, Coleridge, Lamb, Hazlitt, and the Reader of Drama, University of Missouri Press, Columbia 1990. 
Metamorfosi dei

Lumi 10.

L'emergenza

del

sentimento è parte integrante della letteratura, e che non da meno delle forme letterarie, la forma drammaturgica sussume le capacità delle arti plastiche, prestando loro le proprie dinamiche esecutive. Come scrive James Northcote, «take all your ideas from the descriptions of the poets, and all your actions and expressions of the passions from the stage, and you will be a hopeful painter» ${ }^{6}$. Il caso emblematico è quello di Poussin, il quale, notoriamente debitore, forse fin troppo, verso i poeti, deve a quelli specificatamente drammatici non solo la qualità dell'invenzione, ma, di più, la capacità di raffigurare le emozioni, perché le "passioni provengono dal teatro» ${ }^{7}$. Il teatrante, consapevole che espressione, carattere, situazione e azione da lui materializzate sul proscenio sono fenomeni transeunti, ma comunque desideroso di lasciare una traccia indelebile sul pubblico, fa appello alla propria presenza corporea, fino al limite della prevaricazione psicologica (e forse anche fisica!) sullo spettatore:

The Actor must supply the deficiency by violent and decided gestures, actions, and expressions of the countenance, such as may be seen and understood at all distances, and by all the various capacities that compose his audience; and, in as much as his voice is raised above all common modes of speaking, so his expression of the passions and their actions, may be carried beyond all common modes of expression or action, and this may be at times an excuse for his out-stepping the modesty of Nature ${ }^{8}$.

Leigh Hunt, uno dei maggiori critici letterari e teatrali, facendo del periodico «Examiner» il proprio campo di battaglia ideologico, offre un'affermazione radicale, definitiva: «The business of an actor is with the passions, rather than the syllables of his characteres. Let him feel the various emotions of the immortal Hamlet, and let him do his best to impart that feeling to us» ${ }^{9}$.

E basta scorrere le recensioni teatrali, per rendersi conto di come non si parli, per descrivere gli spettacoli, che di passioni, sentimenti, emozioni: «conflict of passions», «burst

\footnotetext{
6. «The Artist», I (1807), n. 9, p. 2.

7. Ibid.

8. Ivi, n. 20, pp. 3-4. Corsivo dell'autore.

9. «The Examiner», XXIV (1808), p. 415.
} 
I linguaggi

delle emozioni

in Inghilterra

tra Sette

e Ottocento:

arte, poesia e teatro

of feelings», «study of tempers and passions», «character of passion», «tide of passions», «variety of passions», «flexibility of emotions», e così via all'infinito, sono le espressioni impiegate per rendere conto del successo o meno di una performance, per cui l'abilità nel personificare le passioni diviene la vera pietra di paragone per giudicare il livello di recitazione degli attori.

Mentre appronta conferenze per la Royal Academy, Fuseli è anche caporedattore per il teatro della «Analytical Review» (dove si firma con la sigla Z.Z.), distribuendo qua e là qualche suo succoso aforisma. Appunto nell' Aphorism $36^{10}$ suddivide l'arte in tre categorie: l'arte epica, correlata al «sublime», che «stupisce»; l'arte storica, correlata alla «verità circostanziata», che «informa» e l'arte drammatica, che riguarda direttamente le emozioni e perciò ci «commuove» ${ }^{11}$. La predilezione di Fuseli proprio per la forma drammatica, lo induce a sottolineare le affinità ideative e compositive tra Shakespeare e Raffaello da un lato, e tra Michelangelo e Milton dall'altro, sulla base dei diversi modi di rapportarsi alle emozioni ( «Things came to Raphael and Shakespeare; Michelangelo and Milton came to things») ${ }^{12}$. Essendo le passioni la spinta propulsiva nei rapporti intersoggettivi, ne consegue l'obbligo di «consider it as the unalterable law of Nature that all your power upon others depends on your own emotions» ${ }^{13}$. E per l'appunto la capacità di rappresentare il confronto/scontro tra i caratteri, determinati dalle rispettive sfere emotive, costituisce la superiorità dell'arte drammatica, di cui Shakespeare è paradigma: «By this radiant recollections of associated ideas, the spontaneous ebullitions of nature, selected by observation, treasured by memory, classed by sensibility and judgment, Shakespeare became the supreme master of passions and the ruler of our hearts» ${ }^{14}$.

\section{Il primato delle emozioni}

In Inghilterra, per tutto il corso del Settecento, e soprattutto nella seconda metà del secolo, il dibattito teorico sulle emo-

10. Gli Aforismi sono raccolti nel terzo volume dell'opera omnia curata da J. Knowles.

11. H. Fuseli, Aphorism 36, in Knowles, cit., vol. III, p. 74.

12. Id., Aphorism 215, ivi, p. 141.

13. Id., Aphorism 200, ivi, p. 137.

14. Id., Lecture III, in Knowles, cit., vol. II, p. 145. 
Metamorfosi dei

Lumi 10.

L'emergenza

del

sentimento zioni si era sviluppato collegandosi prima alla teoria estetica, quindi alla teoria drammaturgica, e infine a entrambe insieme, trattate come due aspetti paralleli e complementari di un medesimo fenomeno, la cui base teorica più semplice era riconducibile alla celebre definizione fornita nel 1649 da Cartesio nel Traité des passions de l'âme. Le passioni sono simultaneamente sensoriali ed emotive, ma anche spirituali, in quanto veicolate dai movimenti dello spirito, che le trasportano attraverso il corpo: «Des perceptions ou des sentiments, ou des émotions de l'âme, qu'on rapporte particulièrement à elle, et qui sont causées, entretenues et fortifiées par quelque mouvement des esprits» ${ }^{15}$. Tale trasporto corporeo degli spiriti animali determina ben identificabili azioni, specificamente riferibili a questa o quella passione: «Ce qui est en l'âme une passion est communément en le corps une action» ${ }^{16}$.

La formula cartesiana era stata recepita in àmbito estetico nell'opera che di fatto apre la strada alla fisiognomica moderna, ovvero la Conférence sur l'impression de differents caractères des passions di Charles Le Brun, del 1698, la cui traduzione inglese, apparsa nel $1701^{17}$, viene subito assunta quale punto di riferimento per le future teorie sia sull'arte, sia sulla recitazione teatrale, soprattutto per la semplice ma operativa distinzione tra passioni "irascibili" e "concupiscibili", con cui Le Brun contrapponeva paura e odio - irascibili, da un lato, a dolore e amore - concupiscibili, dall'altro. Queste emozioni, definite «primarie», vengono veicolate soprattutto dalla mobilità dei muscoli facciali, la quale si ricollega al sistema modale musicale dell'antico teatro greco.

Anche il secondo libro di A Treatese of Human Nature di David Hume (1739), appunto Of the Passions, ${ }^{18}$ articola le emozioni per coppie oppositive, perché ogni emozione presuppone dialetticamente il proprio contrario; e quindi: piacere/ dolore, virtù/vizio, amore/odio, orgoglio/umiltà, desiderio/ avversione, speranza/paura. Hume usa come perfetti sino-

15. R. Descartes, Passions de l'âme (1649), Librairie Philosophique, Paris 1966, p. 84.

16. Ivi, p. 66.

17. Conference of M. Le Brun upon Expression, general and particular, J. Smith, London 1701.

18. D. Hume, A Treatise of Human Nature (1739), a cura di L.A. Selby-Bigge (1896), Oxford University Press, Oxford 1978. 
I linguaggi

delle emozioni

in Inghilterra

tra Sette

e Ottocento:

arte, poesia e teatro nimi i termini impressione, emozione e passione, per quelli che definisce «principi universali», ossia gli elementi costitutivi, essenziali della natura umana ${ }^{19}$, e che pone gerarchicamente al di sopra della ragione. Ricordiamo una delle sue più celebri affermazioni: "Reason is, and ought only to be the slave of the passions, and can never pretend to any other office than to serve and obey them ${ }^{20}$. E proprio giocando sulla relatività e complementarietà tra impressioni, emozioni o passioni, definisce l'attività della mente con una splendida metafora metateatrale: "The mind is a kind of theatre, where several perceptions successively make their appearance; pass, re-pass, glide away, and mingle in an infinite variety of postures and situations» ${ }^{21}$.

3. Retorica delle passioni e arte performativa: fisiognomica, patognomica e caricatura

Il tentativo fallito di Aaron Hill di fondare un'accademia di arte drammatica, risultò invece nel primo dei veri e propri trattati inglesi moderni di retorica e arte performativa. In The Art of Acting, il trasparente sottotitolo pone sul medesimo piano, in base al comune utilizzo delle passioni fondamentali, arti letterarie, arti plastiche e arte drammaturgica: An Essay on General use to the practicers of many elegant Arts, adapted in particular to the Stage $e^{22}$.

Più o meno negli stessi anni, uno scienziato, James Parsons, tiene una serie di conferenze alla Royal Society, che vengono pubblicate nelle Philosophical Transactions della Società con il titolo Human Physiognomy Explained ${ }^{23}$. Quest'opera stabilisce l'antica fisiognomica (risalente addirittura ad Aristotele, e poi nel corso dei secoli, tra i tanti, a Michele Savonarola, Bartolomeo della Rocca, Giovan Battista Della Porta, Jerónimo Cortés, ecc.) come disciplina da trattarsi direttamente all'interno della teoria delle passioni, ad uso, nuovamente,

19. Come Hume conferma nella parziale revisione del Treatise, ossia Enquiries Concerning Human Understanding and Concerning Principles of Morals (1749), a cura di L.A. Selby Bigge e P.H. Nidditch, Oxford University Press, Oxford 1975.

20. D. Hume, Treatise of Human Nature, cit., p. 415.

21. Ivi, p. 253.

22. A. Hill, The Art of Acting (1753), in The Works of the Late Aaron Hill, s.e., London 1779, vol. IV. p. 2.

23. J. Parsons, Human Physiognomy Explained, Royal Society Publishing, London 1749 . 
Metamorfosi dei

Lumi 10.

L'emergenza

del

sentimento

tanto di poeti e artisti, quanto di drammaturghi. La centralità del trattato di Parsons è la sostituzione teorica degli «spiriti animali» di Cartesio con i «muscoli del volto», ripresi da Le Brun, quali vettori fisici delle emozioni, e le illustrazioni che fornisce provengono indifferentemente da testi pittorici o testi drammatici. Sia le teorie sull'arte che quelle sul teatro assorbono velocemente l'idea del movimento muscolare, tant'è che Hogarth nella Analysis of Beauty $(1753)^{24}$, così come Engel nelle Ideen zu einer Mimik (1785-1786) ${ }^{25}$ sottolineano entrambi, per applicazioni diverse (pittura e dramma) il significato profondo della muscolatura facciale. Samuel Foote, drammaturgo e attore, anch'egli autore di un trattato di recitazione, fu uno dei primi ad accogliere la lezione delle conferenze di Parsons; concentrando l'attenzione soprattutto sul sentimento dell'ira, la rabbia, precisa con grande convinzione come «in every degree of this Passion, the Muscles are contracted, and their Force increased, occasioned by the Blood or Spirits being rapidly driven from the other Parts of the Body, to extream and muscular ones» ${ }^{26}$.

Più avanti nel secolo, William Cooke, in uno studio staminale di vera e propria teoria drammaturgica, The Elements of Dramatic Criticism (1775) parla ancora di movimenti muscolari come di ciò su cui deve lavorare particolarmente l'artista, o il poeta, o il drammaturgo: "As a painter, in order to represent the various attitudes of the body, ought to be intimately acquainted with muscular motion, so no less intimately acquainted with emotions, and characters ought a poet to be, in order to represent the various attitudes of the mind ${ }^{27}$. Le passioni che il drammaturgo assegna ai personaggi devono essere avvertite nella profondità dell'anima dall'attore, perché egli possa riprodurle sulla scena, nella mobilità del volto, così come il pittore, per poter copiare i grandi maestri, deve conoscere non meno di loro le leggi dell'espressività e del movimento muscolare ${ }^{28}$.

24. W. Hogarth, The Analysis of Beauty (1753), W. Strahan, London 1772.

25. J.J. Engel, Ideen zu einer Mimik (1785), G. Olms, Hildesheim 1968.

26. S. Foote, A Treatise of the Passions, as far as They Regard the Stage, Corbet, London 1747, p. 12.

27. W. Cooke, The Elements of Dramatic Criticism, G. Kearsly, London 1775, p. 54.

28. Ivi, pp. 197-198. 
I linguaggi

delle emozioni

in Inghilterra

tra Sette

e Ottocento:

arte, poesia e teatro

Sotto l'influenza di questi (e ovviamente altri, trattati) ${ }^{29}$ che divennero subito molto noti ai critici teatrali, si inizia a valutare l'efficacia degli attori in base alla mobilità del volto, espressiva delle emozioni cui devono dare corpo. Molti apologisti dei massimi performers settecenteschi, a partire e finire con i mitici David Garrick a John Philip Kemble, fondavano le lodi sull'estrema «flessibilità dei muscoli facciali», mentre, per contro, molti altri attori, bravissimi, ma un po' meno popolari e osannati, venivano spesso accusati di non saper variare a dovere i propri lineamenti, con quella frequenza che la mobilità delle passioni personificate avrebbe richiesto ${ }^{30} . \mathrm{E}$ da tutto il discorso critico che si snoda sulle riviste specialistiche dell'epoca, nasce una tipica ambiguità, riguardo a chi, tra il pittore e il drammaturgo (e conseguentemente l'attore) influenzi l'altro: sono i teatranti, con la propria retorica facciale, a fornire $\mathrm{i}$ «modelli» agli artisti per i loro ritratti, o sono invece i pittori, a imporre a scrittori di drammi e attori un'ampiamente condivisa pratica accademica, per rappresentare una scena teatrale che vuole apparire realistica?

In ogni caso, per poeti, artisti, drammaturghi e attori, una chiara suddivisione delle emozioni in rigide categorie formali risolveva molti problemi di rappresentazione. A teatro, la fedeltà tecnica a determinate pratiche recitative diviene meno importante del segnale visivo che il «ritratto» del personaggio sulla scena invia agli spettatori. La mimica facciale, la postura, il gesto, significano sia in un quadro sia in una scena l'aderenza del personaggio a questa o quella passione; perciò gli attori possono specializzarsi in questa o quella passione, mentre $\mathrm{i}$ pittori ritrattisti possono lavorare su di un numero limitato di gesti ed espressioni, immediatamente identificabili e riferibili a specifiche, elementari emozioni. E perciò la fisionomia del volto e l'espressività del corpo si impongono come forti stru-

29. Ottima la panoramica proposta in J. Roach, The Player's Passion: Studies in the Science of Acting, University of Delaware Press, Newark-London 1995, cap. V; J. Pascoe, Romantic Theatricality, Cornell University Press, Ithaca-London 1997, passim; M. Pointon, Showing: Women, Possession and Representation in English Visual Culture 1665-1800, Oxford University Press, Oxford-London 1997, capp. III-IV.

30. A tale proposito, non posso che rinviare a due studi fondamentali quali S. West, The Image of the Actor: Verbal and Visual Representation in the Age of Garrick and Kemble, Pinter, London $1991 \mathrm{e}$ W. Worthen, The Idea of the Actor: Drama and the Ethics of Performance, Princeton University Press, Princeton 1984. Cfr. anche C. Corti, $L a$ scena delle Muse cit., pp. 7-60. 
Metamorfosi dei Lumi 10. L'emergenza del sentimento menti di comunicazione, per veicolare l'essenza di intensi momenti drammatici (che a teatro si chiamavano «points») all'interno di un codice interpretabile e apprezzabile tanto da frequentatori di teatri quanto da frequentatori di gallerie d'arte. Gli attori vengono esortati a studiare la pittura per perfezionare la mimica e la gestualità, e parallelamente i critici teatrali possono imputare a un attore che quel particolare gesto o un determinato atteggiamento non sembrano essere stati catturati dalla mano di un bravo artista.

Dopo che la fisiognomica si era imposta come base di valutazione critica per l'analisi dei caratteri umani, la versione inglese dei Phisiognomische Fragmente di Johann Kaspar Lavater, pubblicata in cinque volumi nella traduzione di Thomas Holcroft (significativamente, un drammaturgo!) tra il 1789 e il 1798, come Essays on Physiognomy, venne a dare a questa disciplina uno statuto di indiscutibile (almeno per i tempi!) attendibilità scientifica. La premessa teorica del lavoro monumentale è piuttosto semplice: «Each individual has his character, and every character has a physiognomy proper to it; it is this which gives, if I may express myself, the tone to the look, to the gesture, to the carriage, to the mien, to the gait, to all our movements active or passive ${ }^{31}$.

Muovendo da tale principio elementare, Lavater procede a un'accurata analisi degli specifici tratti del volto, in relazione ai caratteri che questi trasmettono, interpolando osservazioni teoriche sia sulla moralità sia sulla bellezza. L'impianto speculativo di tipo estetico risente palesemente delle teorie portate avanti nel corso del Settecento prima in Germania (dall'Aesthetica di Alexander Gotttlieb Baumgarten, 1750-1758, fino alla kantiana Kritik der Urteilskraft, 1790) e poi in Francia, e parzialmente in Italia. Dopo quello tedesco, è sicuramente il dibattito inglese, con il conte di Shaftesbury, Francis Hutcheson, William Hogarth, Alexander Gerard, Edmund Burke, Henry Home, e molti altri ancora ${ }^{32}$, a determinare una linea di pensiero che va ben oltre gli ancora influenti criteri neoclassici sia nell'analisi della funzione delle arti, sia nel problema del gusto, della psicologia dell'artista, della natura dell'esperienza estetica, il ruolo dell'immaginazione e del sentimento, e più don 1800, vol. IV, p. 229.

32. Cfr. C. Corti, La scena delle Muse, cit., pp. 23-33. 
I linguaggi

delle emozioni

in Inghilterra

tra Sette

e Ottocento:

arte, poesia e teatro

ancora la concezione del bello. Il bello corrisponde - com'è della tradizione platonica e plotiniana - al buono; e perciò, afferma Shaftesbury, «all Beauty is Truth», tutta la bellezza è verità (frase notoriamente cara al poeta Keats), memore, a sua volta, della classica equazione goethiana poesia = vero. $\mathrm{E}$ allora, solo i lineamenti veri, autentici, naturali, costituiscono la bellezza di un volto: «True Features make the Beauty of a Face ${ }^{33}$. Per contro, sostiene Alexander Gerard, «bad affections, expressed at their worst, throw deformity upon the finest features» ${ }^{34}$. Secondo Henry Home, «the External Signs of Emotions and Passions» costituiscono «a natural language», per cui afferma perentorio che «we can read the character of a man in his face» ${ }^{35}$. Più prudente appare la posizione di Hogarth, il quale, pur riconoscendo, nel capitolo Of the Face dell'Analysis of Beauty, che "the face is the index of the mind", avverte comunque come l'apparenza fisica sia il risultato di molti fattori, per cui dubita che la fisiognomica possa essere una disciplina scientificamente affidabile ${ }^{36}$.

Lavater ha invece una fede assoluta nella corrispondenza tra vita interiore e apparenza esteriore, e il suo lunghissimo trattato discute minutamente non solo il significato dei tratti del viso, occhi, naso, soprattutto bocca, ma addirittura dei capelli, e poi della mano, e infine - elemento prezioso quanto quello dei lineamenti del volto ai fini di un'estetica non solo artistica in senso lato, ma specificamente drammaturgica - della voce:

Make a note, I tell the physiognomist, of the human voice - its height, depth, strength, weakness, dullness, clarity, roughness, or charm - its naturalness or artificiality - find out what voices and tones go oftenest together. And if you have a good ear, you will surely manage to deduce the class of forehead, temperament, and character from the voice ${ }^{37}$.

L'influsso di Lavater e in generale della fisiognomica sulla letteratura, l'arte e il teatro, in Inghilterra, è incredibilmente

33. A.A. Cooper Shaftesbury, Characteristics of Men, Manners, Opinions, Times (1727), Grant Richards, London 1900, p. 142.

34. A. Gerard, En Essay on Taste (1759), Scholars Facsimilies and Reprints, University of California Press, Cainesville 1963, p. 44.

35. H. Home, Elements of Criticism, Johnston, London 1765, vol. II, p. 116.

36. Hogarth, Analysis of Beauty, cit., p. 125.

37. Lavater, Essays on Physiognomy, cit., vol. IV, p. 461. 
Metamorfosi dei

Lumi 10.

L'emergenza

del

sentimento

vigoroso. Fuseli supervisionò la traduzione inglese, fornendo materiale illustrativo anche usufruendo dell'aiuto del suo amico poeta e artista William Blake. Nella lista delle sottoscrizioni figuravano artisti e incisori interessati al teatro e alla ritrattistica teatrale, come John Bell, Frances Bourgeois, Francesco Bartolozzi, Thomas Macklin, John Boydell, Samuel de Wilde ${ }^{38 .}$ In misura maggiore rispetto ad altre opere precedenti, la Physiognomy di Lavater diede impulso alla teoria dell'infinita varietà dei caratteri, rivelati dagli atteggiamenti del viso e del corpo. Fuseli si fa promulgatore par excellence della teoria fisiognomica in campo artistico:

[The artist] must make himself master of the muscles, tendons, and ligaments that knit the bones or cover and surround them, their antagonism of action and reaction, their issues, their insertions, and the variety of shapes they assume; when they indicate energy or slackness of action or of frame $[\ldots]$ they furnish the characters of the passions, and by their irritability become the echoes of every impression ${ }^{39}$.

E qui si capisce subito come la teoria fisiognomica fosse lì, bell'e pronta, a uso dei drammaturghi e dei teatranti.

Henry Siddons - mediocre attore figlio della grande attrice Sarah Kemble Siddons, nota ai più come Mrs. Siddons tout-court, ma anche con gli appellativi di «The Divine», e financo «The Queen» - non fu forse neanche un accettabile critico teatrale; e tuttavia si rivelò buon teorico della recitazione, spalleggiando e diffondendo lo studio della fisiognomica in un suo fortunato manuale pratico per l'attore, Practical Illustrations of Rhetorical Gesture ${ }^{40}$, adattamento più familiare del pomposo trattato Ideen zu Einer Mimik di Johann Engel.

Per un altro verso, nei suoi Memoirs, Elizabeth Craven, attrice, capocomica e acuta critica di arti drammaturgiche, considera la fisiognomica assolutamente indispensabile, affinché l'esecutore teatrale possa attualizzare per il pubblico tutta la tessitura emozionale e passionale del testo drammatico:

38. Cfr. J. Carlson, The Theatre of Romanticism, Cambridge University Press, Cambridge-London 1991, passim; F. Burwick, Illusion and the Drama: Critical Theory of the Enlightenment and Romantic Era, Pennsylvania University Press, University Park 1991, cap. II; C. Corti, Shakespeare illustrato, Bulzoni, Roma 1996, cap. I.

39. Lavater, Essays on Physiognomy, cit., vol. II, pp. 321-322.

40. H. Siddons, Practical Illustrations of Rhetorical Gesture, adapted to the English Drama from a Work on the Subject by M. Engel, Phillips, London 1807, pp. 10-11. 
I linguaggi

delle emozioni

in Inghilterra

tra Sette

e Ottocento:

arte, poesia e teatro
Powerful acting requires the physiognomy to be fully seen, else the performance of passions would be lost. All the movements of the soul should be displayed in the physiognomy; the muscles which extend, the veins which swell, the skin which reddens, prove our interior emotions, without which it is impossible to exhibit great talent. It is a part of acting as necessary as that of speaking ${ }^{41}$.

L'attenzione dei teorici drammatici per il movimento, l'azione, l'energia per l'appunto dell'apparato muscolare quali veicoli delle passioni dell'anima, invita a riflettere sul fatto che proprio la mobilità muscolare verrà studiata, tra Sette e Ottocento, di per se stessa, dando così vita a una branca specifica della fisiognomica, che prenderà il nome di patognomica. Fuseli concettualizza così, nei suoi sintetici, drastici, assolutizzanti aforismi, le distinzioni tra la disciplina primaria e la disciplina derivata:

Physiognomy teaches what is homogeneous and what is heterogeneous in forms.

The solid parts of the body are the base of physiognomy, the muscular that of pathognomy; the former contemplates the animal at rest, this in action.

Pathognomy allotts expression to character.

Those who allow physiognomy to regulate the great outlines of character, and reject its minute discriminations, admit a language and reject its elements ${ }^{42}$.

Appunto sulla base delle leggi della patognomica, Fuseli valuta le varie performance dei maggiori attori e attrici inglesi del periodo, che recensisce puntualmente sui fogli della «Analytical Review» (allora prezioso punto di riferimento del pensiero radicale e giacobino in Inghilterra) ${ }^{43}$, dimostrandosi e confermandosi sempre tanto osservatore perspicace quanto critico spietato.

Gli apparati iconografici apposti ai trattati di fisiognomica e patognomica consistono fondamentalmente in schizzi di volti, dove la passione manifestata dal movimento dei mu-

41. A.M. Broadley e L. Melville (a cura di), The Beautiful Lady Craven: The Original Memoirs of Elizabeth Baroness Craven, Bodley Head-Bell \& Cockburn, London-Toronto 1826 , vol. I, pp. 235-236.

42. J. Fuseli, Aphorisms 157-160, in Knowles, cit, vol. III, p. 313. Corsivi dell'autore. 43. Cfr. C. Corti, Rivoluzione e Rivelazione: William Blake tra profeti, radicali e giacobini, Giannini-Liguori, Napoli 2004, cap. I. 
Metamorfosi dei

Lumi 10.

L'emergenza

del

sentimento

scoli facciali viene retoricamente stilizzata, in forme accentuate e stilemi forti. Non c'è di che stupirsi se questi ritratti immaginari divennero fonte di informazione e illustrazione preziosissima per i grandi attori, che a quelli si ispiravano direttamente sul palcoscenico, per raffigurare una determinata emozione.

Teoricamente, le passioni dovrebbero riguardare solo l'arte tragica, in base alla distinzione formulata già alla fine del Seicento tra tragedia come rappresentazione delle passioni e commedia come descrizione degli umori (humours), intendendo per humour, secondo la definizione moderna del migliore commediografo inglese del periodo, William Congreve, "a singular and unavoidable manner of doing or saying anything, Peculiar or Natural to one man only, by which his speech and actions are distinguish'd from those of other men ${ }^{44}$. Tale distinzione resta operativa per tutto il Settecento, e travalica nel teatro romantico, condivisa da attori, drammaturghi e critici teatrali. Una scrittrice di drammi molto apprezzata nel primo Ottocento, nonché finissima (tuttora per noi oggi!) analista e teorica del teatro a lei contemporaneo come Joanna Baillie, per esempio, mentre ricerca nella rappresentazione tragica, in linea con la patognomica, «that variety of fine fleeting emotion which nature in moments of agitation assumes», richiede invece sia agli autori di commedie, sia agli attori comici, di porre attenzione nel «distinguish one man from another by some strange whim or imagination, which is ever uppermost in his thoughts, and influences every action» ${ }^{45}$.

A dire il vero, le illustrazioni apposte ai trattati sulle emozioni, soprattutto quelle relative alla mobilità dei muscoli facciali, proprio perché fornivano modelli di tratti fisiognomici e patognomici molto accentuati, spesso espansi fino ai

44. W. Congreve, Concerning Humour in Comedy (1695), Crowder, Ware \& Payne, London 1773, p. 165. Sebbene legato sia alla filosofia medievale, sia al concetto moderno di personalità, il termine «humour», come lo usa Congreve, indica gli aspetti più fondativi dell'arte comica; la definizione venne poi impiegata quale giustificazione degli elementi eccessivi e grotteschi della commedia contemporanea.

45. J. Baillie, Introduction, in A Series of Plays, Cadell \& Davies, London 1800, pp. 5052. Si veda anche ciò che esprime al riguardo la migliore commentatrice, a oggi, della figura della Baillie, ovvero C. Burroughs, Closet Stages: Joanna Baillie and the Theater Theory of British Romantic Women Writers, University of Pennsylvania Press, Philadelphia 1997, soprattutto nel cap. IV. 
I linguaggi

delle emozioni

in Inghilterra

tra Sette

e Ottocento:

arte, poesia e teatro limiti del grottesco, venivano ampiamente utilizzate quale giornalistica fonte di ispirazione anche per le parti comiche, parimenti offrendo un utile strumento alla critica ufficiale, seriosa, delle commedie rappresentate nei grandi teatri londinesi, Covent Garden e Drury Lane nella season invernale, soprattutto Haymarket nella parentesi estiva. Tant'è che, esattamente come gli attori tragici, anche gli attori comici venivano valutati sulla base della capacità di «disporre»e «alterare» i muscoli del viso, per raffigurare una determinata emozione (evidentemente con finalità ed effetti diversi).

Ebbene, l'esasperazione dei tratti somatici (fisiognomica) e muscolari (patognomica), divulgata dai trattati sulle emozioni umane, sortisce il risultato che così come la critica sulla recitazione tragica ricerca analogie artistiche soprattutto con la pittura a carattere storico, per descrivere l'arte dell'attore tragico, parallelamente la critica della recitazione comica si avvale di riferimenti alla caricatura. L'Abbé Le Blanc fu uno dei primi a sottolineare le affinità espressive e comunicative (elettive?) tra la recitazione comica e quell'arte figurativa - tanto cara agli inglesi! - della caricatura, lanciata da Hogarth e proseguita a fine Settecento da esimi artisti incisori quali Thomas Rowlandson e James Gillray. Leggiamo questa ironica, un po' mordace critica del peraltro bonario connaisseur-des-arts francese pellegrino in Inghilterra:

The English, if you'll permit me to use a term of painting, which can alone express my idea, love caricaturas; they are more struck with a large face and a great nose, designed by Callot, than with a noble and graceful countenance, trac'd by Correggio. [...] The more an actor finds caricatura in his part, the more he thinks there ought to be of it, in his action; and thus, he endeavours to express the humour of it, more by the grimaces of his face than the proper modulation of his voice ${ }^{46}$.

Anche i critici appaiono spesso perplessi davanti a spettacoli - non solo commedie, ma anche tragedie - dove un eccesso di «coloratura» nella mimica facciale trasforma il personaggio, tanto quello «passionale» quanto quello «caratteriale», in una maschera ridicola o capricciosa. Molti deprecano la

46. J.B. Le Blanc, Letters on the English and French Nations, translated from the French, London-Dublin 1747, vol. II, pp. 6-7. 
Metamorfosi dei

Lumi 10.

L'emergenza del sentimento tendenza della recitazione ad assumere tonalità «caricaturali» che, se relativamente accettabili nella commedia, lo sono molto meno nella tragedia. E tuttavia, anche nelle recensioni positive, è un dato di fatto che il metalinguaggio mutuato dalla caricatura impregna di sé il linguaggio critico. Come dimostra, per esempio, una nota di John Hunt sulla recitazione contemporanea, stimolata dall'apertura della season nel novembre del 1808: gli attori erano sì delle «caricature», delle maschere esorbitanti, eppure, "their faces smirked upon us from the superb engravings with a sidelong satisfaction» ${ }^{47}$.

Sembra dunque lecito concludere che le teorie patemiche, fisiognomiche e patognomiche, con l'idea sottostante dell'infinita varietà di espressioni, penetrano tutto il discorso critico-teorico prima settecentesco e poi ottocentesco sulle arti plastiche e sull'arte drammatica, considerate affini sulla base di una comune appartenenza alla rappresentazione sia delle passioni (arte tragica), sia delle caratteristiche individuali (arte comica): entrambe, comunque, emozioni; e che, in un caso o in un altro, sembrano essere gli attori, nella straordinaria fama raggiunta dai teatranti in Inghilterra, tra Sette e Ottocento, i più idonei a fornire «ritratti», di portata semantica universale quelli tragici, modellati sulle passioni staminali, di contenuto più soggettivo quelli comici, determinati da peculiarità di atteggiamenti psichici. Perché solo sul palcoscenico «that combination of beauties arising from voice, air, gesture, and expression», fa sì che solo «a great Performer rivets the attention, and electrifies the heart» ${ }^{48}$. 
Metamorfosi dei

Lumi 10.

L'emergenza del

sentimento
Musique et émotion au XVIII siècle: quelques regards sur une question sans réponse

Pierre Saby niques de ma vie ${ }^{1}$ (1935-1936):

Je considère la musique, par son essence, impuissante à exprimer quoi que ce soit: un sentiment, une attitude, un état psychologique, un phénomène de nature, etc. L'expression n'a jamais été la propriété immanente de la musique. La raison d'être de celle-ci n'est d'aucune façon conditionnée par celle-là. $\mathrm{Si}$, comme c'est presque toujours le cas, la musique paraît exprimer quelque chose, ce n'est qu'une illusion et non une réalité. C'est simplement un élément additionnel que, par une convention tacite et invétérée, nous lui avons prêtée, imposée, comme une étiquette, un protocole, bref, une tenue, et que, par accoutumance ou inconscience, nous sommes arrivés à confondre avec son essence ${ }^{1}$.

Il formulait ainsi de façon radicale une conception de l'art musical comme art autonome, c'est-à-dire ne désignant que lui-même, et pour lequel il revendiquait une liberté d'exercice pleine et entière, indépendante de toute nécessité fonc- 
Metamorfosi dei

Lumi 10.

L'emergenza

del

sentimento tionnelle liée à l'expression d'autre chose que lui-même. Il prolongeait et développait ainsi, dans le contexte du courant «néo-classique» dont il fut un temps l'un des tenants, des idées qui avaient été esquissées ou même véritablement formulées par certains musicographes de la fin du XVIII ${ }^{\mathrm{e}}$ siècle, en anticipation de ce que l'on a pu appeler au XIX ${ }^{\mathrm{e}}$ les théories «formalistes» de la musique. Par exemple, Michel-PaulGuy de Chabanon, musicien et homme de lettres français, qui écrivait quant à lui en 1779:

La Musique par son essence n'est point un Art d'imitation: elle se prête à imiter autant qu'elle le peut; mais cet office de complaisance ne peut la distraire des fonctions que sa nature même lui impose. L'une de ces fonctions nécessaires, est de varier à chaque instant ses modifications, d'allier dans le même morceau le doux et le fort, le trainant et le détaché, l'articulation fière, \& celle qui est affectueuse. Cet art, ainsi considéré, est d'une inconstance indisciplinable: tout son charme dépend de ses transformations rapides $[\ldots]^{2}$.

Ou encore, La Cépède, en 1785:

Quelques vives que soient les peintures offertes par une symphonie, il sera aisé de voir qu'elles ne seront que des images vagues. L'on pourra être ému par le tableau de toutes les passions, éprouver tous les sentimens, être témoin pour ainsi dire de toutes les affections; mais il sera bien difficile, \& presque toujours impossible de reconnaître le fond des événements par lesquels on sera ému $[\ldots]^{3}$.

On peut esquisser un commentaire de ces fragments dans plusieurs directions complémentaires, qui nous serviront à introduire notre propos. On voit tout d'abord que la fonction expressive de la musique pose question de longue date, mais que si certains s'attachent à la discuter, c'est qu'elle a fait de plus longue date encore l'objet d'une forme de dogme, lié de façon plus générale à la doctrine des arts comme imitation de la nature, idée sur laquelle nous reviendrons. On voit d'ailleurs, dans les mêmes textes, que si le «sentiment», la «passion», l' «affection», le caractère expressif lié à des

2. M.P.G. de Chabanon, Observations sur la musique et principalement sur la métaphysique de l'art, Pissot, Paris 1779, pp. 75-76.

3. B.G.É. de Laville, comte de La Cépède, La Poétique de la musique, Imprimerie de Monsieur, Paris 1785, p. 330. 
Musique et émotion au Xvil' siècle: quelques regards sur une question sans réponse concepts inscrits dans le champ moral ou celui des affects («articulation fière, affectueuse»), constituent un aspect central du discours, cela s'inscrit dans une problématique plus large, qui est celle de l'imitation de la nature en général, laquelle concerne aussi les phénomènes physiques observables («peintures», «images», «événements»). Chez La Cépède comme chez Chabanon, dans d'autres pages, c'est d'abord l'imitation de la nature physique qui fait l'objet de la réflexion critique. Chabanon explique ailleurs, en prenant des exemples qui évoquent à l'évidence les célèbres Stagioni de Vivaldi - le chant des oiseaux, le murmure des ruisseaux, l'orage - que l'identification, la prétendue reconnaissance par l'auditeur des phénomènes imités est une chimère, et ne procède en réalité que de la pure convention. Pour La Cépède, à défaut de pouvoir peindre la nature physique observable par l'homme et extérieure à lui, la musique semble rester capable d' «imiter» la nature humaine (l'intériorité) et donc d'évoquer, faire reconnaître, puis éventuellement faire «éprouver» des affects; dans le propos de Chabanon, on comprend que la musique peut, certes, émouvoir mais que, d'une part, elle le fait par la seule mise en ouvre de ses moyens techniques, strictement endogènes (les jeux d'intensité - le «doux», le «fort»-, «l'articulation»...) et non en imitant un modèle extérieur à elle, d'autre part, qu'elle séduit par son caractère insaisissable, ses transformations, la mobilité de ses caractères. Sur cet aspect, nous serons également amené à revenir.

Comme on le sait, la source du concept d'imitation de la nature dans les arts se trouve chez les anciens grecs, Platon d'abord, puis Aristote. À titre de rappel, on peut en redire ici quelques mots, qui nous paraissent indispensables à la cohérence de notre propos, en particulier pour ce qui concerne la conception platonicienne de la musique:

- un réseau de correspondances et de circulation des concepts met en communication l'harmonie céleste (l'âme de l'univers, régie par une harmonie numérique), l'harmonie de l'individu (âme humaine), l'harmonie de la cité (organisation politique);

- la musique terrestre, dont l'harmonie est régie par le nombre, est et doit être le reflet de l'harmonie céleste. 
Metamorfosi

dei

Lumi 10.

L'emergenza

del

sentimento
Elle est pourvue d'un ethos qui change selon le mode, le rythme, le genre. De ces prémisses procède l'idée de la musique imitant les passions et porteuse d'expression. Mais au-delà, elle a le pouvoir d'agir sur l'âme humaine, et il y a des musiques bénéfiques, car leur mode, surtout, est porteur d'ethos positif : les jeunes gens élevés au contact de musiques belles et pures s'imprégneront des vertus qui en émanent et contribueront naturellement à l'harmonie de la cité. Inversement, certaines musiques doivent être bannies de la cité, voire, interdites aux poètes, parce que porteuses, donc génératrices, de passions «négatives» (Livres III, VI, X de La République).

Ces conceptions trouvèrent un relais et un écho manifestes dans les études antiques de la Renaissance, notamment en Italie, au sein des Accademie (l'idée que la musique a du pouvoir sur les humains est manifestement pour quelque chose, au-delà de la jouissance physique et intellectuelle que procurent les arts, dans l'intérêt que les pouvoirs politiques, donc les princes - florentins, mantouans, par exemple -, portent à la musique. Ce qu'écrit encore Rousseau sur le sujet dans la seconde moitié du XVIII ${ }^{\mathrm{e}}$ siècle dans son Dictionnaire de Musique atteste de la manière dont il comprend Platon, et suggère que, peut-être, l'idée interpelle encore les penseurs:

Platon ne craint pas de dire qu'on ne peut pas faire de changement dans la Musique qui n'en soit un dans la constitution de l'État, et il prétend qu'on peut assigner les Sons capables de faire naître la bassesse de l'âme, l'insolence et les vertus contraires $[\ldots]^{4}$.

Dans l'Italie de la fin du $\mathrm{xvI}^{\mathrm{e}}$ siècle, la quête de la musique des anciens grecs débouche sur l'invention du style de la monodie accompagnée, et la chimère de la restauration de la tragédie antique aboutit à la création d'un genre nouveau, le dramma per musica. La déclamation chantée, par opposition à la polyphonie savante, est alors explicitement pensée comme vecteur de la sensibilité, de l'affectivité, par exemple

4. J.-J. Rousseau, article «Musique», Dictionnaire de Musique, in CEuvres complètes, a cura di B. Gagnebin e M. Raymond, Gallimard, Paris 1959-1995, 5 voll., vol. V, pp. 915-926. 
Musique et émotion au XVIII siècle: quelques regards sur une question sans réponse

par Giulio Caccini, qui écrit dans la Préface de son recueil $L e$ Nuove Musiche, paru en 1601 à Florence ${ }^{5}$ :

Lo affetto in chi canta altro non è che per la forza di diverse note, e di vari accenti co'l temperamento del piano, e del forte una espressione delle parole, e del concetto, che si prendono à cantare atta à muovere affetto in chi ascolta.

(Le pathétique chez celui qui chante n'est autre qu'une certaine expression du mot et de sa signification, apte à émouvoir la passion chez qui l'écoute; et cela est par la puissance de certaines notes, l'énergie de certains accents que permet la modulation du «piano» et du «forte»).

Le genre du dramma per musica (ou favola per musica: Peri, Caccini, Monteverdi, Gagliano...) constitue l'un des lieux d'exercice privilégiés de ce pouvoir de la musique, dans le cadre d'un spectacle dramatique et musical, voué à la représentation des passions, dans la filiation de la Mimésis aristotélicienne. L'évolution du genre au XVIII ${ }^{\mathrm{e}}$ siècle, envisagée dans ses grandes lignes, consacre une dichotomie poétique et musicale de plus en plus marquée entre le recitativo (dialogue, progrès de l'action...) et l'aria, dévolue à l'expression de l'affect (l'action s'arrête), et potentiellement terrain favorable à la cristallisation de stéréotypes, qui finissent presque par rendre les arie interchangeables, par famille ou types d'affect (vendetta, lamento, di sogno, jubilo..., qui font le genre de l'opera seria au XVIII ${ }^{\mathrm{e}}$ siècle), sur la base des moyens choisis par le compositeur pour «traduire » en musique un affect particulier, et - à peu de choses près - unique d'un bout à l'autre de l' aria (la partie centrale de l'aria col da capo apporte éventuellement quelques nuances, mais on termine l'aria en en reprenant la $1^{\text {re }}$ section): choisi parmi beaucoup d'autres exemples possibles, l'aria d'Irene, à la fin du premier acte du Bajazet d'Antonio Vivaldi (1735, Carnaval de Vérone), se signale ainsi par son caractère plutôt homogène: en dépit du texte du libretto évoquant la lutte de deux sentiments, la mise en musique traduit surtout l'agitation du personnage, secoué par les émotions.

5. G. Caccini, Le Nuove Musiche, Marescotti, Firenze 1701. 
Metamorfosi dei

Lumi 10.

L'emergenza

del

sentimento

96
Cette aria appelle, au demeurant, au moins deux commentaires distincts:

Tout d'abord, elle met en lumière de manière aiguë la problématique inhérente à toute musique vocale ayant prétention à «exprimer», ou à valoriser le poème: par exemple, les vocalises virtuoses développées sur le «a» médian de «battaglia» sont évidemment déclenchées par l'idée d'agitation violente qui est portée par le mot, que l'on songe à une bataille réelle ou, par analogie, au conflit des émotions. Mais si le mot est le moteur du geste musical, l'apparition de l'émotion en constituant l'objectif, l'objet produit, sitôt que l'on s'attarde sur la voyelle en question (et on s'y attarde longuement), se dégage inévitablement de sa sujétion au mot pour, en réalité, prendre le pouvoir, et devenir un objet prioritairement musical. Dans l'écriture de Vivaldi, le mot est d'ailleurs, à plusieurs reprises, coupé pour une respiration ou une cadence, etc.

On comprend aussi, à partir de là, quel problème particulier peut poser le développement d'une virtuosité remettant en question la prééminence de la poésie, pour les amateurs de musique attachés à la fonction du chant comme vecteur privilégié de l'émotion. Certes, l'émotion pourra naître du seul contact avec la virtuosité pure, mais cette approche est au XVIII ${ }^{\mathrm{e}}$ siècle encore majoritairement anachronique, et elle reste étrangère, notamment, à la sensibilité française. Non contents de condamner les excès spectaculaires des chanteurs italiens, les hommes de culture français sont très réticents, au début du siècle, devant le développement de la musique purement instrumentale (genres de la sonate, du concerto), dont les italiens sont, à nouveau, les promoteurs (la musique pour cordes, en particulier), et ce dans la mesure où cette musique, détachée au moins en apparence de toute idée poétique, est suspectée de ne rien imiter et, par conséquent, de ne véhiculer aucune émotion. C'est une grande partie du propos, par exemple, de Jean Laurent Lecerf de la Viéville (1674-707), dans son ouvrage intitulé Comparaison de la musique italienne et de la musique française, paru en $1704^{6}$. C'est aussi dans cette perspective qu'il faut comprendre le mot attribué au philosophe Bernard Le Bovier de Fontenelle:

6. J.L. Lecerf de La Viéville de Fréneuse, Comparaison de la musique italienne et de la musique française, Foppens, Bruxelles 1704. 
Musique et émotion au XVIII' siècle: quelques regards sur une question sans réponse
«Sonate, que me veux-tu?», de même que la déclaration de François Couperin, dans la Préface de son Premier Livre de clave$\operatorname{cin}(1713)^{7}$ : «J'avouerai de bonne foi que j'aime beaucoup mieux ce qui me touche que ce qui me surprend». La problématique «virtuosité instrumentale vs chant et expression» constitue jusque tard dans le siècle l'un des sujets développés par la critique musicale naissante, dans le «Mercure de France», puis «Le Journal de Paris» notamment, à propos des musiciens et des œuvres entendues au Concert Spirituel parisien, la virtuosité spectaculaire des instrumentistes étant parfois mise en cause, parce que perçue comme étrangère à une démarche d'expression des affects...

L'héritage des anciens, notamment de Platon, concernant

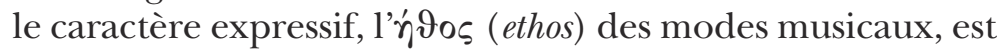
à nouveau au cœur d'un autre débat, très actif notamment en France, concernant la question du tempérament musical.

On se contentera ici de rappeler que le terme de tempérament désigne la façon, variable au cours de l'histoire de la musique européenne, d'accorder les instruments à clavier, de façon à rendre le système sonore naturel compatible avec la pratique musicale. En effet:

- les calculs développés à partir du principe de la division des cordes vibrantes (dont l'origine est pythagoricienne) permettent, de façon combinatoire, de déterminer la grandeur exacte des intervalles de la musique (intervalles «purs»), du plus simple qui est en même temps le plus grand, c'est-à-dire l'octave (division de la corde par 2), en passant par les consonances (quinte, tierce), jusqu'aux dissonances et aux intervalles les plus petits (demi-ton).

- or, le système sonore naturel ainsi matérialisé est un système «ouvert»: si, partant sur un clavier d'un son grave donné (un $u t$ ), on accorde successivement une série de cordes de quinte en quinte «pure», on finit par obtenir des intervalles trop grands, et les rapports d'intervalles de tierce, et surtout d'octave ne sont pas exacts ${ }^{8}$. La question

7. F. Couperin, Pièces de clavecin [...], Premier Livre, chez l'Auteur, et Boivin, Paris 1713.

8. Précisément: la douzième quinte pure, si\#, qui devrait se trouver dans un rapport d'octaves justes avec l'ut initial, se trouve en réalité trop haute d'un comma. On trouve un exposé clair des prémisses théoriques et de la pratique courante du 
Metamorfosi dei

Lumi 10.

L'emergenza

del

sentimento

des tierces peut être résolue de diverses façons, mais il est indispensable pour la pratique musicale que les octaves soient parfaitement justes, faute de quoi toute pratique polyphone serait impossible: il faut donc diminuer les quintes, de façon à obtenir des octaves justes: c'est ce que l'on appelle le tempérament. Les tempéraments pratiqués dans toute la musique ancienne, jusque dans le XVIII $^{\mathrm{e}}$ siècle, sont dits inégaux, parce que l'on diminue certaines quintes plus que d'autres. De là, une réalité objective: toutes les tonalités ne «sonnent» pas de la même façon, parce que les intervalles de même nature qui les composent (par exemple do-sol en do majeur, et mi-si en $m i$ majeur), alors qu'ils ont la même position et la même fonction dans les deux gammes, ne sont pas exactement de même grandeur acoustique; de même, le résultat est que certains demi-tons sont plus grands que d'autres, et que les «grands» et les «petits» demi-tons ne sont pas distribués de manière identique dans les différentes gammes ou tonalités.

La conséquence de l'inégalité des tempéraments est le développement, particulièrement en France, de systèmes que l'on pourrait qualifier de «psycho-acoustiques», ou «psycho-esthétiques", attribuant à chaque tonalité, ou à certaines d'entre elles, une sorte d'ethos particulier, un caractère expressif lié à un certain type d'émotion (en France, le traité Les passions de l'âme de René Descartes est toujours au XVIII siècle une lecture influente, au même titre que le Discours sur la méthode, et l'on sait que le philosophe entend aborder la question des émotions en physicien plutôt qu'en moraliste: l'émotion serait le résultat d'un processus dont l'origine est de nature physique). En 1722, Jean-Philippe Rameau consacre à la question un chapitre de son Traité de l'harmonie $e^{9}$. Des auteurs divers produisent ce type de système - plus ou moins détaillé, concernant un nombre plus ou moins grand de tonalités, avec entre eux certaines convergences et aussi des divergences... dont on peut observer, à des degrés divers, la

tempérament inégal vers la moitié du XVIII ${ }^{\mathrm{e}}$ siècle dans les Élémens de musique théorique et pratique, suivant les principes de M. Rameau di J. Le Rond d'Alembert, Bruyset, Lyon 1752 et 1762, chapitre VII, pp. 39-50.

9. J.P. Rameau, Traité de l'harmonie réduite à ses principes naturels, Ballard, Paris 1722, livre II, chapitre XXIV, «De la propriété des modes \& des tons». 
Musique et émotion au XVIII' siècle: quelques regards sur une question sans réponse

mise en œuvre dans les compositions musicales (le ton de fa \#, réputé particulièrement malaisé et inutilisable parce que trop grinçant, est visité par Bach dans ses deux grandes Passions (Johannes Passion et Matthäus Passion) au moment où l'évangéliste rapporte le reniement et les remords de l'apôtre Pierre, à la fin de la vocalise déployée sur les mots «weinete bitterlich» («il sortit, et pleura amèrement»). Les compositeurs continuèrent assez longtemps à faire référence dans leurs compositions à ce type de conceptions, alors même que s'était engagée la bataille pour le «tempérament égal». Le choix d'une tonalité principale susceptible de porter une émotion, un affect en particulier procède, au fond, d'une conception statique de la peinture des émotions (pour un morceau, un affect), conception accordée à la mise en œuvre d'architectures musicales simples (formes en deux sections, par exemple), au sein desquelles les changements de ton (les modulations) ne se font que pour visiter des tons très proches du ton principal, souvent un seul ton voisin, le plus proche. L'évolution du langage musical en général (textures, formes, procédés d'écriture, notion émergente de thème et surtout de développement sur les thèmes, requiert des possibilités de modulations plus fréquentes, moins prévisibles, plus éloignées, et appelle inévitablement une démarche d'égalisation du tempérament (toutes les quintes atténuées de la même manière), de façon à pouvoir utiliser tous les tons sans dissonances criantes). Rameau fut, en France, le principal promoteur du tempérament égal, arguant que les différences entre les intervalles sont, au fond, inappréciables, et que l'expression d'un morceau vient de la façon dont aménage les changements de ton, plutôt que du choix initial de la tonalité principale. Ses adversaires objectent que le langage musical ne peut que perdre ainsi toute sa faculté à peindre, et à faire éprouver sentiments, passions, émotions... Ainsi Jean-Jacques Rousseau, dans l'article «Ton» de son Dictionnaire de Musique:

Ces Tons diffèrent entre eux [...] par les diverses altérations des Sons \& des Intervalles, produites en chaque Ton par le Tempérament [...].

De-là naît une source de variétés \& de beautés dans la Modulation. De-là naît une diversité \& une énergie admirable dans l'expression. De-là naît enfin la faculté d'exciter des sentimens différens avec des Accords semblables frappés 
Metamorfosi

dei

Lumi 10.

L'emergenza

del

sentimento

en différens Tons. [En un mot, chaque Ton, chaque Mode, a son expression propre qu'il faut savoir connoître, \& c'est-là un des moyens qui rendent un habile Compositeur maître, en quelque manière, des affections de ceux qui l'écoutent [...].

C'est pourtant de cette agréable \& riche diversité que M. Rameau voudroit priver la Musique, en ramenant une égalité \& une monotonie entière dans l'Harmonie de chaque Mode, par sa règle du Tempérament $[\ldots]^{10}$.

L'argumentation de Rameau (pour résumer: c'est de l'agencement des différents changements de ton à l'intérieur d'un morceau que naissent l'expression et le sentiment, et non du choix de la tonalité principale) anticipe, en quelque sorte, celle des théoriciens de la fin du siècle concernant l'inanité de la fonction prétendûment «imitative» de la musique ${ }^{11}$. Le débat sur cette question a été actif tout au long du siècle chez les musicographes et esthéticiens français: Du Bos ${ }^{12}$, Batteux $^{13}$, Estève ${ }^{14}$, par exemple; puis encore d'Alembert, Rousseau, Diderot...: il s'agit d'intégrer la musique à un système général des Beaux-Arts imitant la nature. À défaut de pouvoir attribuer à la musique des pouvoirs d'imitation de la nature physique - le monde observable - similaires à ceux de la peinture ou de la poésie, on s'attache à lui reconnaître la prééminence dans le domaine de l'imitation des affects. Nous convoquerons ici Jean-Jacques Rousseau, auteur d'un très beau texte auquel il semble avoir tenu particulièrement, puisqu'après l'avoir écrit pour l'article «Imitation, en musique» de l'Encyclopédie, il le reproduisit une première fois dans son Dictionnaire ${ }^{15}$, ainsi que dans son Essai sur l'origine des langues $^{16}$.

10. J.-J. Rousseau, Dictionnaire de Musique, cit., Article «Ton», pp. 516-517.

11. Notamment (voir supra, au début de notre étude) M.P.G. de Chabanon, Observations sur la musique et principalement sur la métaphysique de l'art, Pissot, Paris 1779 et De la musique considérée en elle-même et dans ses rapports avec la parole, les langues, la poésie et le théâtre, Pissot, Paris 1785, et B.G.É. de Laville, comte de La Cépède, La Poétique de la musique, Imprimerie de Monsieur, Paris 1785.

12. J.B. Du Bos (abbé), Réflexions critiques sur la poésie et sur la peinture, Mariette, Paris 1719.

13. C. Batteux, Les Beaux Arts réduits à un même principe, Durand, Paris 1746.

14. P. Estève, L'Esprit des Beaux-Arts, Bauche, Paris 1753.

15. Article «Imitation». Voir supra.

16. Essai sur l'origine des langues, où il est parlé de la mélodie et de l'imitation musicale, 
Musique et émotion au XVIII' siècle: quelques regards sur une question sans réponse

C'est un des grands avantages du musicien, de pouvoir peindre les choses qu'on ne saurait entendre, tandis qu'il est impossible au peintre de représenter celles qu'on ne saurait voir; et le plus grand prodige d'un art qui n'agit que par le mouvement est d'en pouvoir former jusqu'à l'image du repos. Le sommeil, le calme de la nuit, la solitude et le silence même, entrent dans les tableaux de la musique. On sait que le bruit peut produire l'effet du silence, et le silence l'effet du bruit, comme quand on s'endort à une lecture égale et monotone, et qu'on s'éveille à l'instant qu'elle cesse. Mais la musique agit plus intimement sur nous, en excitant par un sens des affections semblables à celles qu'on peut exciter par un autre; et comme le rapport ne peut être sensible que l'impression ne soit forte, la peinture, dénuée de cette force, ne peut rendre à la musique les imitations que celleci tire d'elle. Que toute la nature soit endormie, celui qui la contemple ne dort pas, et l'art du musicien consiste à substituer à l'image insensible de l'objet celle des mouvemens que sa présence excite dans le cœur du contemplateur. Non-seulement il agitera la mer, animera la flammes d'un incendie, fera couler les ruisseaux, tomber la pluie et grossir les torrens; mais il peindra l'horreur d'un désert affreux, rembrunira les murs d'une prison souterraine, calmera la tempête, rendra l'air tranquille et serein, et répandra de l'orchestre une fraîcheur nouvelle sur les bocages. Il ne représentera pas directement ces choses, mais il excitera dans l'ame les mêmes sentimens qu'on éprouve en les voyant ${ }^{17}$.

En un raccourci certes trompeur, mais nous semble-t-il néanmoins significatif, autorisons-nous à mettre en regard de ce passage ce qu'écrivait Chabanon en 1779:

On chante, on note les sons que l'on a dans la tête: ces sons ne sont pas l'expression de la chose, ils sont la chose même ${ }^{18}$.

In fine, ainsi que nous le suggérions en ouverture de ces quelques notes, le débat sur la nature et le périmètre possible de l'imitation en musique conduira logiquement à la contes-

rédigé à partir de 1755, publié par Du Peyrou, à qui Rousseau avait confié le manuscrit. Voir OEuvres posthumes de J.-J. Rousseau, vol. III, Genève 1781, pp. 211-327.

17. Chapitre XVI, «Fausse analogie entre les couleurs et les sons». Rééd.: texte établi et présenté par Jean Starobinski, Gallimard, coll. «folio essais», Paris 1990, pp. 106-107.

18. M.P.G. de Chabanon, Observations sur la musique, cit., p. 146. 
Metamorfosi

dei

Lumi 10.

L'emergenza

del

sentimento

102 tation du dogme lui-même, contestation qui se trouve d'ores et déjà plus qu'à l'état latent sous la plume de Chabanon...

On terminera ce survol en évoquant deux aspects de la musique du dernier XviII ${ }^{\mathrm{e}}$ siècle, entretenant tous deux des relations avec la question de l'émotion. Le premier point touche à l'esthétique musicale classique dans l'une de ses manifestations, peut-être, caractéristiques : l'exemple choisi sera tiré de l'œuvre de Christoph Willibald Gluck. Le second point que nous évoquerons renvoie à ce que l'on a coutume de nommer en allemand le courant empfindsam (en français, «sensible»), notamment mis en œuvre, de façon emblématique, jusque tard dans le siècle, dans certaines pièces de l'un des fils de Johann Sebastian Bach, Carl Philipp Emanuel, mais à l'occasion aussi dans la musique de ses illustres contemporains, par exemple Wolfgang Amadeus Mozart.

En 1762, Christoph Willibald Gluck donne à Vienne, sur un poème de Ranieri de' Calzabigi, Orfeo ed Euridice, le premier de ses opéras «réformés»: par ce terme, il faut entendre «épurés», débarrassés de tous les stéréotypes, excès, déviances ou pratiques jugées déplacées par Gluck, de l'opera seria tel qu'il avait évolué sous le règne des chanteurs vedettes.

Au-delà de la notoriété de l'ouvrage, on s'est parfois interrogé quant à la nature de l'aria célèbre «Che farò senza Euridice», le caractère apaisé, serein, voire, lumineux, en tout cas au début, du morceau, semblant à certains commentateurs tout à fait inadapté à la situation (Orfeo vient de perdre son épouse Euridice, définitivement prisonnière du royaume des morts), et ne reflétant pas, il s'en faudrait de beaucoup, l'émotion alors éprouvée par le personnage, et potentiellement attendue chez le spectateur. La plastique de la mélodie, sa fluidité, la tonalité en mode majeur, la couleur de l'orchestre à cordes... ne semblent en effet véhiculer aucun désespoir, a fortiori aucune espèce de révolte. Or, on peut avoir de cet état de fait une lecture éventuellement plus pertinente en rétablissant la solidarité du couple constitutif «récitatif/aria», l'aria en question étant précédée d'un assez long récitatif. En effet dans le récitatif, la démarche de peinture, d'expression des émotions qui agitent Orfeo est explicitement mise en ouvre : sentiment tragique et expression du pathos y sont suscités avec beaucoup de maîtrise et d'efficacité par le compositeur. Le couple récitatif/aria apparaît alors 
Musique et émotion au XVIII' siècle: quelques regards sur une question sans réponse comme indissociable, la section déclamée concentrant en son sein l'intensité, voire, la violence des émotions, et l'aria fonctionnant comme exutoire et comme lieu de purification, d'épuration de la passion, via la plastique sereine de la mélodie et les autres caractères musicaux qui lui sont associés. Dans un cas comme celui-ci, l'aria n'est plus le lieu de l'épanouissement ou du déchaînement de l'émotion, mais celui de son épuration, de sa canalisation; la perfection du chant devient le moyen par lequel on peut se rendre maître de l'émotion... et de la beauté naît une émotion nouvelle, qui transfigure toutes les autres. La doctrine baroque selon laquelle, en quelque sorte, «l'œuvre émeut, donc elle est belle» se trouverait alors comme renversée: «l'œuvre est belle, donc elle émeut». C'est, en quelque sorte, l'essence de la dualité baroquisme/classicisme en art qui se trouve concentrée dans un couple d'objets constitutifs essentiels au genre de l'opéra: le récitatif, l' aria ${ }^{19}$.

Quant aux manifestations du courant Empfindsam dans la musique de la fin du XviII ${ }^{\mathrm{e}}$ siècle, on en trouve maint exemple dans la production de tous les compositeurs majeurs du temps, spécialement d'origine germanique. L'un des traits les plus caractéristiques des musiques qui procèdent de ce courant est bien celui de la mobilité expressive, ce en quoi nous rejoignons le contenu de certains des textes que nous avons évoqués plus haut. Cette mobilité, érigée parfois en caractère générique de l'art musical (rappelons Chabanon: «mais cet office de complaisance [l'imitation] ne peut la distraire des fonctions que sa nature même lui impose. L'une de ces fonctions nécessaires, est de varier à chaque instant ses modifications [...]. Cet art, ainsi considéré, est d'une inconstance indisciplinable : tout son charme dépend de ses transformations rapides»), peut être lue comme le signe d'un passage: passage d'une esthétique du sentiment

19. Une problématique similaire peut être observée, à notre sens, dans le Don Giovanni de Mozart (Acte II, sc. 10): la tempête et le tourbillon émotionnels du récitatif «In quali eccessi, ô Numi» se déversent, mais surtout s'apaisent, se «classicisent», dans l'aria qui suit, «Mi tradi quell'alma ingrata», dont les virtuoses volutes largement déployées ont souvent été jugées déplacées, et comprises comme de vaines concessions à une virtuosité vocale dépourvue de consistance dramatique. Or il ne saurait s'agir d'une inconséquence de la part d'un compositeur parvenu définitivement au sommet de son art de musicien de théâtre, et qui composa les deux morceaux en question dans un geste second, pour la reprise viennoise de l'ouvrage en 1788, après la création à Prague en 1787. 
Metamorfosi

dei

Lumi 10.

L'emergenza

del

sentimento

104 à une esthétique de la sensibilité (c'est-à-dire, au fond, de l'aptitude au sentiment), à une esthétique de l'expressivité plus que de l'expression. Au cœur de cette esthétique du «sensible», la mobilité du langage devient en soi une source d'émotion, et la mobilité des affects possibles, dont il ne subsiste parfois que des signes furtifs, peut conduire à une forme d'indétermination du sentiment, alors même que le langage présente tous les symptômes d'une expressivité brûlante: ne sont plus très éloignées les conceptions romantiques de la musique (notamment instrumentale, qui ne fait pour l'auditeur l'objet d'aucune pré-détermination signifiante), musique comme art de l'obscur et de l'intériorité insondable, musique non plus accompagnatrice du drame ou medium de l'émotion, mais musique comme drame, musique comme émotion. Dans certaines pièces de la fin du XviII ${ }^{\mathrm{e}}$ siècle, l'un des signes les plus visibles de cette approche semble être la mise en cause de la forme, de l'architecture du morceau, critère pourtant central de toute démarche artistique classique. Nous donnerons de cet aspect des choses deux exemples nous semble-t-il significatifs, et laisserai ainsi le dernier mot aux musiciens: on peut, dans l'œuvre de Carl Philipp Emmanuel Bach, écouter dans cette perspective le rondo pour clavier en do mineur extrait du cinquième des recueils «Für Kenner und Liebhaber» (1785): alors même que la forme rondo est a priori une forme claire et simple, celle-ci se dilue dans une succession de volte-face expressives qui finissent par en détruire les contours. Une impression similaire se dégage, dans un contexte tout différent pourtant, de la première aria du personnage de Cherubino, dans le premier acte des Nozze di Figaro de Wolfgang Amadeus Mozart (1786): «Non so più cosa son, cosa faccio...", chante d'abord le jeune adolescent, comme s'il portait, au-delà des émois sensuels et amoureux de son âge, le trouble d'une génération d'artistes en proie, au moins pour les plus «sensibles» d'entre eux, au vertige devant les abîmes grands ouverts que commence à leur laisser entrevoir une façon nouvelle de sentir, de penser, de composer la musique: la première partie de l'aria se conforme à un schéma traditionnel ternaire (ABA), virtuellement appelé à faire fonction de section initiale, possiblement, d'une forme en deux grandes sections, mais la fin du morceau avec son alternance finale de phrases suspendues, d'élans et de silences, évacue à peu près, au niveau perceptif en tout cas, 
Musique et émotion au XVIII siècle: quelques regards sur une question sans réponse toute référence à un discours formel clair, en même temps que s'accroît l'intensité éruptive d'une sensibilité mobile, à fleur de peau, hésitante entre plusieurs affects.

C'est ainsi, on le comprend, toute l'aventure de l'art musical au travers de cet «âge classique» que constituent, pour nous, les $\mathrm{XVII}^{\mathrm{e}}$ et $\mathrm{XVIII}^{\mathrm{e}}$ siècles réunis, qui se dévoile dans la perspective de cette question cruciale: la mise en œuvre de l'émotion, des émotions, par le truchement du geste compositionnel... Depuis les enthousiasmes fondateurs des madrigalistes du premier Seicento, érigeant en dogme la quête de la traduction des affects, et explorant presque méthodiquement les moyens de la mener à bien, jusqu'aux fécondes incertitudes des musiciens des Lumières, frayant finalement les voies inexplorées d'un pèlerinage dont ils sentent bien que par la musique, il les ramènerait à l'obscur ${ }^{20}$, si jamais ils en avaient quitté l'empire, l'histoire du classicisme musical, de son épiphanie et de sa maturation, nous apparaît le plus souvent comme celle de la lutte organique et fraternelle que se livrent, dans le champ des œuvres du génie, exigence rationnelle d'une part, séductions des sens et caprices de l'émotion, d'autre part. Et les certitudes revendiquées sur le sujet, au début du xxe siècle, par Stravinsky ou certains de ses confrères nous apparaissent, finalement, presque aventureuses, du fait même de leur posture simplificatrice.

20. Par la musique vers l'obscur. Essai sur la musique bourgeoise et l'éveil d'une conscience allemande au XVII siècle et aux origines $d u$ XIX , est le titre d'une thèse de doctorat ès lettres, publiée à tirage limité à Marseille en 1942, par le poète, romancier et dramaturge Marcel Beaufils, qui fut professeur d'esthétique au Conservatoire National Supérieur de Musique Paris de 1949 à 1969. L'ouvrage a été réédité aux éditions Robert Laffont sous le titre Comment l'Allemagne est devenue musicienne (Paris 1983). 

Parte terza

Sentimento

e politica

aA 

Metamorfosi dei

Lumi 10.

L'emergenza del

sentimento
Coltivare le emozioni tra solitudine e socialità. Gli esempi di Shaftesbury e Zimmermann

Elisa Leonzio nella storia delle idee

Affermata come una sorta di topos nella germanistica del XX secolo di lingua tedesca e internazionale - in Italia varrebbe da solo l'esempio di Croce ${ }^{1}$-, l'influenza di Shaftesbury sulla letteratura e sulla filosofia tedesca del Settecento ha però sofferto e in parte continua ancora oggi a soffrire di una lettura parziale.

A segnarla è stata in primis l'analisi offerta da Dilthey, che nella seconda edizione (1922) della sua monumentale biografia di Schleiermacher, presenta Shaftesbury come la principale fonte del teologo accanto a Spinoza ${ }^{2}$.

Altrettanto importante sul piano della storia delle idee è l'interpretazione che di Shaftesbury dà Cassirer nel suo celebre scritto del 1932 Filosofia dell'illuminismo ${ }^{3}$. Cassirer in-

1. B. Croce, Shaftesbury in Italia, «La critica», 23 (1925), pp. 1-27.

2. W. Dilthey, Leben Schleiermachers, in Id., Gesammelte Schriften, voll. 13-14, Vandenhoeck \& Ruprecht, Göttingen 2006; trad. it. La vita di Schleiermacher, 2 voll., Liguori, Napoli 2008.

3. E. Cassirer, Die Philosophie der Aufklärung, Meiner, Hamburg 2007; trad. it. La filosofia dell'illuminismo, La Nuova Italia, Firenze 1999. 
Metamorfosi dei

Lumi 10.

L'emergenza

del

sentimento

110 siste sul nesso presente tra estetica e teologia nel pensiero di Shaftesbury che verrebbe esemplificato dall'analogia, presente nel Sensus communis e nel Soliloquio, tra cosmo e opera d'arte, ovvero tra l'attività creatrice di Dio e quella dell'artista. Shaftesbury, a detta di Cassirer, offrirebbe in essi una rilettura, pur radicalmente modificata, dell'idea platonica dell'arte come imitazione, presentando l'artista, novello Prometeo, come un secondo artefice, che nell'opera d'arte crea un microcosmo secondo le medesime leggi con le quali Dio ha creato il macrocosmo ${ }^{4}$. E queste leggi egli le coglie mediante un'intuizione intellettuale, che a sua volta è di nuovo tema di ascendenza platonica. La creazione artistica diventa quindi qualcosa di soggettivo, frutto dell'immedesimazione dell'artista nel genio universale, cioè in Dio. In questo richiamo al genio, nel ricorso al mito di Prometeo e nell'assenza di norme prescrittive per la realizzazione dell'opera d'arte, Cassirer vede l'elemento di modernità del pensiero di Shaftesbury, che traghetta l'estetica e l'arte tedesca dal classicismo al romanticismo.

Le argomentazioni di Cassirer avevano alla propria base la convinzione che si debba rintracciare nel platonismo la fonte principale della filosofia shaftesburiana e questa lettura sembrerebbe in realtà essere avvalorata da una tradizione interpretativa che era già settecentesca. Basti pensare che la fortuna di Shaftesbury in Germania è legata al giudizio che della sua filosofia diede Leibniz nel 1715 a commento dell'uscita, nel 1714, della seconda edizione delle Characteristicks of Men, Manners, Opinions and Times, la raccolta delle proprie opere voluta e organizzata dallo stesso Shaftesbury nel 1711. Nella Histoire critique de la république des Lettres Leibniz sottolinea la consonanza tra il proprio pensiero e quello di Shaftesbury soprattutto per quel che riguarda il concetto di armonia e della sua filosofia loda in particolar modo «il ritmo del discorso, il nuovo platonismo e la maniera di argomentare attraverso continue domande $»^{5}$.

4. A.A. Cooper Shaftesbury, Soliloquy, in Id., Characteristics of Men, Manners, Opinions, Times, Cambridge University Press, Cambridge 2004, p. 93.

5. G.W. Leibniz, Éloge critique des æuvres de Milord Shaftesbury par Mr. le Baron de Leibnits, communiqué par Mr. Coste, «Histoire critique de la république des Lettres», $\mathrm{X}$ (1715), pp. 306-327, qui p. 323: «Le tour du Discours, [...] le Platonisme nouveau, la manière d'argumenter par interrogations». 
Coltivare le emozioni tra solitudine e socialità. Gli esempi di Shaftesbury e Zimmermann

Questo accostamento al platonismo, questa vera e propria "platonizzazione" del suo pensiero, ha allo stesso tempo giovato e nuociuto a Shaftesbury. Gli ha giovato nel momento in cui la cultura tedesca ha cercato un'alternativa all'imperante materialismo e meccanicismo di stampo francese e ha creduto di trovarla nel mondo inglese, in quella linea di discendenza che da Platone arriva a Shaftesbury attraverso il platonismo di Cambridge. Gli ha però nuociuto nel momento in cui in Germania ha preso gradualmente piede una corrente antiplatonica, che verrà superata solo con la pubblicazione dell'edizione Zweibrücker dell'opera platonica tra il $1781 \mathrm{e}$ il 1787. Se si ripercorre brevemente la storia delle traduzioni tedesche di Shaftesbury si notano sostanziali somiglianze nell'andamento: numerose sono le traduzioni e le citazioni di stralci dalle sue opere fino alla metà del Settecento, che rappresenta apparentemente l'apice della sua fortuna. Dopodiché occorre attendere la fine degli anni Settanta e gli anni Ottanta per la traduzione integrale delle Characteristicks e la riscoperta di Shaftesbury.

Gli studi più recenti, da circa quindici anni a questa parte, tendono a mettere in dubbio la lettura platonica di Shaftesbury e puntano piuttosto sullo stoicismo come modello alternativo $^{6}$. Nonostante questo spostamento di interpretazione dal paradigma platonico a quello stoico, lo studio della ricezione di Shaftesbury è stato però solo parzialmente corretto. L'epoca privilegiata per saggiare l'influenza della filosofia shaftesburiana è adesso l'illuminismo, ma si considera prevalentemente la prima metà del Settecento. Dopodiché si accetta l'idea che egli cada in disgrazia, come Platone, fino a essere recuperato, come Platone, negli ultimi decenni del secolo. Ma a questo punto lo sguardo degli studiosi è nuovamente rivolto a ciò che sta per venire, al romanticismo $\mathrm{e}$ all'idealismo.

La tesi che si vorrebbe qui sostenere è invece quella di una sopravvivenza di Shaftesbury anche, e proprio, nella seconda metà del secolo, insistendo sull'influenza che le sue concezioni antropologiche e la sua psicologia morale hanno esercitato sulla filosofia popolare tedesca e sulle sue principali ramificazioni, l'antropologia e la psicologia empirica. Certo, egli non

6. Cfr. H.-G. Dehrmann, Shaftesburys stoischer Sokratismus, "Aufklärung», 22 (2010), pp. 77-103. 
Metamorfosi dei

Lumi 10.

L'emergenza

del

sentimento

viene più tradotto per circa un trentennio e scompare dalle discussioni ufficiali, ma la sua presenza rimane viva in ambiti più privati (si pensi alla corrispondenza di Mendelssohn e Hamann) e divulgativi, in conformità a quella tendenza alla diffusione del sapere in sedi extra-accademiche che è caratteristica fondamentale della filosofia popolare tedesca.

La riflessione sulle emozioni e sul loro rapporto con la solitudine e la socialità rappresenta un terreno ideale in cui mettere alla prova questa tesi, ripercorrendo alcune delle posizioni di Shaftesbury sull'argomento e cercandone poi le tracce nell'opera di Zimmermann, prototipo del medicofilosofo del tardo Settecento e autore di alcuni saggi e di uno studio monumentale sulla solitudine.

\section{Solitudine e socialità: che cosa è naturale?}

Nel 1730, in occasione della sua lezione inaugurale Sulla natura sociale dell'uomo in qualità di professore di morale all'università di Glasgow, Francis Hutcheson, figura di spicco dell'illuminismo scozzese e principale esponente, accanto a Shaftesbury, della filosofia del moral sense, sottolineava come fosse in atto una "disputa sul senso in cui la vita sociale può essere considerata naturale per l'uomo» ${ }^{7}$. E spiegava:

Anche se scrittori recenti hanno dichiarato che questa socievolezza [sociabilitas] è la fonte di quasi ogni nostro dovere, nessuno sembra aver spiegato in modo sufficientemente chiaro, in generale, quali cose possono essere propriamente definite naturali per l'uomo, che cosa sia poi questa nostra socialità [socialitas] e infine quali parti della nostra natura ci rendano adatti e inclini alla società, che sia essa società civile o una società non soggetta all'autorità umana ${ }^{8}$.

7. F. Hutcheson, Inaugural Lecture on the Social Nature of Man, in T. Mautner (a cura di), Hutcheson: Two Texts on Human Nature, Cambridge, Cambridge University Press, Cambridge 1993, pp. 124-147, p. 134: «Dispute about the sense in which this social life can be said to be natural to man». L'originale latino del testo, De naturali hominum socialitate. Oratio inauguralis, è invece contenuto in F. Hutcheson, Collected Works, Olms, Hildesheim 1990, vol. VII, pp. 171-200.

8. Ivi, p. 127: «And even if most recent writers have declared this sociality [sociabilitas] to be the source of almost every duty, they nevertheless do not seem to have sufficiently explained, in general, what things are properly called natural to man, nor, more specifically, what this sociality [socialitas] of ours is, nor, finally, which parts of our nature render us fit for and inclined towards society, be it civil society or a society not subject to human authority». Rispetto alla traduzione di Mautner appare però utile accogliere la proposta di Piirimäe e Schmidt, che riservano sociality al latino socialitas e ricorrono invece a sociability per rendere sociabilitas in 
Coltivare le emozioni tra solitudine e socialità. Gli esempi di Shaftesbury e Zimmermann

Con queste parole Hutcheson dava voce a uno dei problemi sentiti come più urgenti nell'Europa del XVIII secolo, ossia quello di legittimare specifiche forme di convivenza e di governo e il relativo complesso di leggi che ne era alla base facendo ricorso alla loro maggior o minore naturalezza. La celebre definizione aristotelica che vedeva nell'uomo uno zoon politikon, un animale politico, aveva cominciato a esser messa in discussione almeno a partire dal XVI secolo e ciò rischiava di minare la sovranità politica e incrinare la ragion di stato. Era in gioco la possibilità stessa di una legge naturale e di una giustizia e giurisprudenza universali. La risposta di Grozio a questo problema era stata la teorizzazione di un $a p$ petitus societatis, ossia di un desiderio universalmente diffuso in tutti gli uomini di vivere pacificamente in uno stato per garantirsi la sopravvivenza. Nel corso del XviII secolo questa soluzione viene variamente ridiscussa, affermata, negata o comunque riformulata ${ }^{9}$. Da un lato vi è chi la respinge, come Rousseau che, sulla scorta di Mandeville, propende per un progressivo sviluppo della sociabilità, ma ne nega il carattere innato, e chi invece, come appunto Hutcheson, ma anche Shaftesbury, la interpreta come una spontanea inclinazione dell'uomo a essere benevolo nei confronti dei propri simili e a cercarne la compagnia. In particolare, Hutcheson ne sottolinea il carattere disinteressato, contrapponendosi a interpretazioni invece più utilitaristiche, incentrate cioè sull'interesse del singolo e sull'amor di sé.

In questo quadro si comprende facilmente l'interesse e la varietà di interpretazioni spesso antagonistiche che nel Settecento suscitò anche il polo opposto alla socievolezza e società, quello della solitudine ${ }^{10}$. Nelle sue manifestazioni originarie la solitudine era un fenomeno di stampo principalmente religioso: l'ascesi e poi il monachesimo significavano, infatti, "essere soli con Dio". Con il trascorrere dei secoli, e in particolare in epoca rinascimentale, si compie il passaggio a una forma mondanizzata di solitudine: fa la sua comparsa

modo da conservare la differenza terminologica presente nell'originale. Cfr. E. Piirimäe e A. Schmidt., Introduction: Between Morality and Anthropology - Sociability in Enlightenment Thought, «History of European Ideas», 41 (2015), n. 5, pp. 571-588.

9. Cfr. U. Im Hof, Das gesellige Jahrhundert. Gesellschaft und Gesellschaften im Zeitalter der Aufklärung, Beck, München 1982.

10. Cfr. K. Wittler, Einsamkeit. Ein literarisches Gefühl im 18. Jahrhundert, «DVjs», 87 (2013), n. 2, pp. 186-216. 
Metamorfosi

dei

Lumi 10.

L'emergenza

del

sentimento

114 l'immagine dell'artista che proprio nella solitudine può esplicare la propria forza creatrice.

Con l'illuminismo si compie un ulteriore passo in questo processo di mondanizzazione: al centro della speculazione filosofica settecentesca vi è, infatti, l'uomo come singolo individuo, e l'interesse va quindi sempre più alla sfera della vita privata, al singolo considerato di per sé, isolato dalla società o inserito in un contesto sociale ristretto, quello della casa e della famiglia. Emblematica di questa scoperta dell'individualità è la fortuna di un romanzo come il Robinson Crusoe, modello esemplare dell'uomo che nella solitudine esplica le proprie capacità e si realizza pienamente. Ci troviamo qui di fronte alla descrizione di una solitudine "attiva", "produttiva" e dalla forte valenza pedagogica: nella solitudine l'uomo può realizzare liberamente la propria personalità senza dover sottostare a condizionamenti esterni. D'altra parte, però, l'individuo è impensabile senza il contesto sociale in cui vive e senza un fondamento (naturale o artificialmente indotto è, come si è visto, motivo di discussione) che sancisca i suoi obblighi verso i concittadini e verso lo stato.

Emergono in questo contesto una serie di interrogativi così riassumibili: per natura l'uomo è socievole o è solitario? E che cosa contribuisce maggiormente alla sua felicità e piena realizzazione, la solitudine o la vita in comune? E, ancora, è possibile che l'uomo sia spontaneamente incline a qualcosa che però gli nuoce? Si può essere soli e asociali pur nella società? E al contrario, essere socievoli e praticare la socialità pur nella solitudine? ${ }^{11}$

Sembrano paradossi, e in qualche caso poco più che giochi di parole, eppure alcune di queste formulazioni sono state davvero utilizzate nel corso del Settecento. L'esempio più significativo è quello di Kant che nella Idee zu einer allgemeinen Geschichte in weltbürgerlicher Absicht ${ }^{12}$ (Idea per una storia universale in prospettiva cosmopolitica), pubblicata nel novembre del 1784 sulla «Berlinische Monatsschrift», uti-

11. Sull'enorme interesse mostrato dagli illuministi per il tema della solitudine si veda fra gli altri W. Haug, Programmierte Einsamkeit. Zur Anthropologie eines narrativen Musters, in A. e J. Assmann (a cura di), Einsamkeit. Archäologie der literarischen Kommunikation, vol. VI, Fink, München 2000, pp. 59-75.

12. I. Kant, Idee zu einer allgemeinen Geschichte in weltbürgerlicher Absicht, in Id., Kant's Gesammelte Schriften, Akademie Ausgabe, Königlich Preußische Akademie der Wissenschaften, Berlin 1900 sgg., vol. VIII, pp. 15-32. 
Coltivare le emozioni tra solitudine e socialità. Gli esempi di Shaftesbury e Zimmermann

lizza l'espressione «ungesellige Geselligkeit des Menschen», la «socievolezza asociale dell'uomo». Secondo Kant l'uomo prova al contempo due inclinazioni antagoniste: da un lato è propenso all'aggregazione sociale, in quanto riconosce che solo in essa egli può sviluppare appieno la propria natura di uomo; dall'altro sente una forte inclinazione ad accentuare la propria singolarità e a isolarsi, poiché prova l'istinto di affermare la propria volontà in modo assoluto e incontra in ciò la resistenza degli altri, così come del resto oppone lui resistenza, quando sono gli altri a voler affermare in modo assoluto la loro. Kant parla in questo caso di una «ungesellige Eigenschaft», una qualità asociale che fa parte anch'essa della natura umana. È proprio questa resistenza, però, il motore che risveglia le altre forze nell'uomo, che lo scuote dallo stato di torpore e pigrizia e lo induce a cercare il proprio posto, e possibilmente un posto di spicco, in mezzo agli altri uomini, sviluppandosi pienamente e passando così da uno stato di rozzezza al dispiegamento della cultura. Spiega Kant:

Senza quelle qualità, peraltro indegne, dell'asocialità e della resistenza che da essa scaturisce e che ciascuno inevitabilmente incontra nell'affermazione egoistica di sé, tutti i talenti resterebbero per l'eternità in una forma embrionale, confinati in una vita da pastori dell'Arcadia, in uno stato di perfetta armonia, soddisfazione e amore reciproco: gli uomini, mansueti come le pecore che accudiscono, conferirebbero alla propria esistenza un valore di poco superiore a quanto ne possiede il loro bestiame; non saprebbero riempire il vuoto della creazione riconoscendo il suo scopo, come natura razionale. Si renda quindi grazia alla natura per l'intolleranza nei confronti dell'altro, per la superbia che spinge a una competizione malevola, per la sete di possesso e di dominio che non viene mai soddisfatta ${ }^{13}$.

13. Id., p. 21: «Ohne jene an sich zwar eben nicht liebenswürdige Eigenschaften der Ungeselligkeit, woraus der Widerstand entspringt, den jeder bei seinen selbstsüchtigen Anmaßungen notwendig antreffen muß, würden in einem arkadischen Schäferleben bei vollkommener Eintracht, Genügsamkeit und Wechselliebe alle Talente auf ewig in ihren Keimen verborgen bleiben: die Menschen, gutartig wie die Schafe, die sie weiden, würden ihrem Dasein kaum einen größeren Werth verschaffen, als dieses ihr Hausvieh hat; sie würden das Leere der Schöpfung in Ansehung ihres Zwecks, als vernünftige Natur, nicht ausfüllen. Dank sei also der Natur für die Unvertragsamkeit, für die mißgünstig wetteifernde Eitelkeit, für die nicht zu befriedigende Begierde zum Haben oder auch zum Herrschen! Ohne sie würden alle vortreffliche Naturanlagen in der Menschheit ewig unentwickelt schlummern». Sul tema si veda A. Belwe, Ungesellige Geselligkeit: Kant: Warum die 
Metamorfosi dei Lumi 10.

L'emergenza del sentimento
Il sovvertimento è qui completo, in quanto sono gli istinti più bassi e malevoli a essere naturali; è naturale la mancanza di socievolezza, che però è conditio sine qua non per la nascita della società. Se l'asocialità è naturale e positiva, non altrettanto può dirsi invece della solitudine "arcadica", da idillio pastorale, che Kant deride. Essa è un tipo di solitudine cosiddetta passiva, dell'uomo contento, o che si accontenta, che indugia e gode nella e della propria condizione appartata e indulge alle fantasticherie e al sentimentalismo. Ci si trova qui davanti a quella che, in un tentativo di classificazione delle forme della solitudine, potrebbe essere definita la solitudine sentimentale: quella solitudine, cioè, che non ha per scopo né il dispiegamento delle proprie potenzialità né la vicinanza o l'avvicinamento al divino, bensì è fine a se stessa e si alimenta promuovendo sentimenti che nascono dalla contemplazione della quieta natura.

Di questa ambivalenza nella valutazione delle inclinazioni solitarie o sociali dell'uomo e della manifestazione storica di queste inclinazioni in un campionario che va dall'eremitaggio alle società moderne è piena la pubblicistica del Settecento, in particolar modo di lingua tedesca. Colpisce, in Germania, la diffusione di periodici dai titoli emblematici, quali, per citarne solo alcuni, Der Einsiedler (L'eremita), Der Anachoret (L'anacoreta) e Der Gesellige (L'uomo sociale). Vi si trovano, alternati, giudizi favorevoli od ostili alla solitudine, in maniera fra l'altro non sempre coerente con l'orientamento che si sarebbe portati a desumere dai titoli delle riviste, che quindi, evidentemente, non esprimevano tanto un valore programmatico, quanto la volontà di far riflettere il lettore.

È interessante leggere, per il suo valore emblematico, il Bilancio dell'anno 1740 che un lettore invia al settimanale morale Der Einsiedler e che fornisce un elenco delle ore che l'uomo ha dedicato durante l'anno alle diverse attività:

Ore dedicate al sonno: 2555.

Ore dedicate al movimento del corpo: 800.

Ore dedicate al diletto dello spirito: 195.

Ore dedicate ad attività sociali inevitabili: 100.

Ore dedicate a mangiare e bere: 730 . 
Coltivare le emozioni tra solitudine e socialità. Gli esempi di Shaftesbury e Zimmermann
Ore dedicate al prossimo e al miglioramento del suo stato: 2100.

Ore dedicate a combattere la povertà: 90 .

Ore dedicate a Dio: 1400.

Ore che si sarebbero potute utilizzare in modo più fruttuoso: 600.

Ore che mancano all'appello: $130^{14}$.

Per quanto questo bilancio possa apparire ingenuo e suscitare il sorriso, la problematica che esso implicitamente presenta è fondamentale: solitudine (intesa in questo caso come raccoglimento in preghiera, come tempo trascorso con Dio) e socialità sono conciliabili? A quali attività, se si esclude il soddisfacimento dei bisogni elementari, è preferibile, più etico e più conforme alla natura dell'uomo dedicarsi? Nessuna risposta viene offerta qui e resta imprecisato quale fosse «il miglior uso» che si sarebbe potuto fare di quelle 600 ore. Ma il problema non poteva essere posto in maniera più immediata.

\section{Teoria delle emozioni: Shaftesbury e lo stoicismo}

Shaftesbury (1671-1713) si pone agli albori della discussione settecentesca su socievolezza e solitudine e lo fa a partire da un'accurata disamina degli impulsi e delle emozioni dell'uomo.

Alla base della nota teoria shaftesburiana del moral sense vi è la convinzione che esistano nell'essere umano inclinazioni naturali, spontanee e disinteressate, alla socialità e alla promozione del bene comune. Shaftesbury le definisce affezioni naturali (natural affection). Accanto a esse si trovano, per citare ancora la sua formulazione, le affezioni egoistiche ( self affection), che tendono invece al benessere individuale. Anche le affezioni egoistiche, per quanto orientate all'individuo e non alla collettività, sono comunque positive in quanto aiutano a preservare in ogni uomo l'istinto di sopravvivenza, e divengono dannose solo se sono troppo accentuate o si

14. Schluß der Rechnung vom Jahr 1740, «Der Einsiedler», ii, $1757^{2}$, p. 9: «Auf der Schlaf verwandt: 2555. Auf die Bewegung des Leibes: 800. Zur Gemüthsergötzung: 195. In Gesellschaft, die ich nicht vermeiden können: 100. Auf Speis und Trank: 730. Auf den Nächsten und dessen Besserung: 2100. Der Armuth abzuhelfen: 90. Zum Dienst meines Gottes: 1460. Nutzbarer hätten können angewendet werden: 600 . Stunden die wo nicht gar verlohren, doch vermisset werden: 130. Stunden des Jahres: 8760». 
Metamorfosi dei Lumi 10. L'emergenza del sentimento

presentano in numero eccessivo. L'agire morale si basa dunque su una personalità dalla struttura armonica in cui tutti gli impulsi naturali benevoli nei confronti di se stessi, della specie e della società cooperano.

Assolutamente negative sono invece le affezioni innaturali, che non perseguono né il benessere pubblico né quello del singolo e risultano anzi massimamente dannose per entrambi ${ }^{15}$. Shaftesbury, ricorrendo alla nomenclatura stoica, chiama questo tipo di affezione passion (passione).

Esiste dunque una linea di demarcazione chiarissima fra affections e passions e solo queste ultime generano uno stato patologico i cui effetti sono immediatamente visibili prima di tutto sul corpo e poi sul comportamento umano. Non è un caso, in tal senso, se Shaftesbury nella Lettera sull'entusiasmo si sofferma sulla passione della malinconia, vista come disturbo che nasce dai fermenti del corpo, dalle sue eruzioni e che sul corpo, prima ancora che sulla mente, esercita il suo influsso ${ }^{16}$.

Va detto subito che il problema del commercium mentis et corporis non riveste ancora per Shaftesbury la centralità che invece assumerà nel prosieguo del secolo. Per lui, più che altro, è importante opporsi con forza a qualsiasi riduzionismo fisiologico e nel Solilogy (1710), creando un parallelismo tra l'ipotetico visitatore di un negozio di orologi e il filosofo, spiega:

Se analogamente un filosofo, dedicandosi allo studio della natura umana, scoprisse unicamente quali effetti ciascuna passione produca sul corpo, quale cambiamento d'aspetto o di forma determini, e in quale differente maniera influisca sulle membra e i muscoli, ciò potrebbe abilitarlo a dare consigli a un anatomista o a un pittore, ma non al genere umano e a se stesso, perché, in base a tale esame, egli non avrebbe tenuto conto dell'azione e dell'energia reali del suo soggetto e non avrebbe considerato l'uomo

15. A.A. Cooper, conte di Shaftesbury, An Inquiry Concerning Virtue or Merit in Id., Characteristics of Men, Manners, Opinions, Times, Cambridge University Press, Cambridge 2004, p. 200: «private or self affections», «natural affections» e «unnatural affections».

16. Shaftesbury, A letter concerning enthusiasm to my Lord ***** in Id., Characteristics of Men, Manners, Opinions, Times, cit., p. 9. 
Coltivare le emozioni tra solitudine e socialità. Gli esempi di Shaftesbury e Zimmermann in quanto uomo reale e agente umano, ma in quanto orologio o comune macchina ${ }^{17}$.

Shaftesbury insiste dunque sul fatto che al filosofo debba interessare l'influenza delle passioni sulla condotta dell'uomo, ossia sul piano delle relazioni interpersonali, più che sul corpo. Tuttavia, il suo continuo far ricorso a una terminologia legata alla dietetica e alla medicina dimostra un significativo intreccio del discorso etico e di quello scientifico.

In stretto rapporto con la sua teoria delle affezioni e ancora una volta ispirandosi alla filosofia stoica, Shaftesbury introduce, sempre nel Soliloqy, il tema della solitudine, la aiskesis, in cui egli vede lo strumento attraverso cui l'uomo può tenere sotto controllo le passioni affinché non prendano il sopravvento sulle affezioni:

A seconda che mi dominino oppure no, che differiscano o meno l'una dall'altra, le passioni influenzano il mio carattere e mi rendono altro da me stesso e dagli altri. Devo perciò trovare il sistema per correggere e migliorare tale condizione, riflettendo opportunamente sul modo in cui funzionano i miei moti interiori, quando sono guidati da affezioni che dipendono così tanto da timore e presunzione. Esaminando i vari rivolgimenti delle passioni, le loro modulazioni, deviazioni e rivoluzioni interne, dovrò senza dubbio giungere a una migliore comprensione del cuore umano, e a formulare giudizi più corretti sia sugli altri che su me stesso ${ }^{18}$.

17. Shaftesbury, Soliloqy, in Characteristics of Men, Manners, Opinions, Times cit., p. 131: «Should a philosopher, after the same manner, employing himself in the study of human nature, discover only what effects each passion wrought upon the body, what change of aspect or feature they produced and in what different manner they affected the limbs and muscles, this mighty possibly qualify him to give advice to an anatomist or a limner but not to mankind or to himself, since, accordingly to this survey, he considered not the real operation or energy of his subject, nor contemplated the man, as real man and as a human agent, but as a watch or common machine». Cfr. Scritti politici e morali, a cura di A. Taraborrelli, Utet, Torino 2007, p. 291.

18. Ivi, p. 132: «These passions, according as they have the ascendancy in me and differ in proportion with one another, affect my character and make me different with respect to myself and others. I must, therefore, of necessity find redress and improvement in this case, by reflecting justly on the manner of my own motion as guided by affections which depend so much on apprehension and conceit. By examining the various turns, inflections, declensions and inward revolutions of the passions, I must undoubtedly come the better to understand a human breast, and judge the better both of others and myself»; trad. it. pp. 291-292. 
Metamorfosi dei

Lumi 10.

L'emergenza

del

sentimento

Il metodo offerto per tenere sotto il giogo le passioni e permettere il pieno dispiegamento delle affezioni sociali appare però in paradossale contrasto con ciò che così si vuole promuovere, in quanto la solitudine sembrerebbe essere la condizione antisociale per eccellenza. La soluzione a questo apparente paradosso può venire, io credo, da un'analisi socio-psicologica che metta in rapporto forma dialogica, linguaggio e pensiero. Proprio nel Soliloqy, il cui motto di apertura è un verso della prima satira di Persio che recita «Nec te quaesiveris extra» ${ }^{19}$, Shaftesbury riflette sulla sua concezione del pensiero. Punto di partenza del ragionamento è una critica alle tendenze letterarie del suo tempo: sempre più autori pubblicano libri molto pretenziosi, ma, se ben analizzati, in fondo privi di contenuto. Come correttivo, anzi come vero e proprio viatico, Shaftesbury suggerisce loro di parlare in privato, restare con se stessi e praticare l'introspezione conversando con se stessi ${ }^{20}$. La parola utilizzata per quest'ultimo concetto è self-converse, di cui risulta subito evidente il duplice significato: da un lato essa rimanda all'introspezione, alla inspection, come la chiama anche Shaftesbury, ossia al convertere lo sguardo dentro di sé; dall'altro conserva un forte legame con il conversare, il parlare con gli altri. Come sia possibile parlare con se stessi diviene allora la domanda fondamentale dell'intero testo. Shaftesbury si richiama a tal proposito alla tradizione greca e in particolare al concetto del daimon socratico:

Si potrebbe dimostrare, infatti, che è sempre una forma di sacrilegio o di empietà disdegnare la compagnia di un ospite così divino [...] Ciascuno di noi ha un paziente in se stesso, noi stessi siamo l'oggetto della nostra pratica e poi diventiamo legittimi praticanti quando, ritirandoci nella nostra coscienza, possiamo scoprire una certa duplicità dell'anima e dividere noi stessi in due persone ${ }^{21}$.

19. Persio, Satire, traduzione e note di E. Barelli, Rizzoli, Milano 1998, p. 123: «Non cercare te fuori di te».

20. Cfr. Shaftesbury, Soliloqy, cit., p. 75; trad. it. p. 213.

21. Ivi, p. 77: «It would infallibly be proved a kind of sacrilege or impiety to slight the company of so divine a guest. [...] We had each of us a patient in ourself [ sic], we were properly our own subject of practice, we then became due practitioners when, by virtue of an intimate recess, we could discover a certain duplicity of soul and divide ourselves into two parties»; trad. it. p. 214. 
Coltivare le emozioni tra solitudine e socialità. Gli esempi di Shaftesbury e Zimmermann

$\mathrm{Al}$ dialogo viene dunque attribuita una funzione euristica. Il dialogare con gli altri o con il sé che si separa e diviene altro, genera il sapere, che nel Soliloqy si profila come conoscenza dell'uomo. Ed è solo quando impara a osservare se stesso e gli altri, come si è visto, che l'individuo diviene in grado di trasformare le affezioni naturali in un sapere morale consapevole e controllare le passioni.

Dietro alla metafora del sacrilegio compiuto da chi rifiuti la compagnia del demone si celano due dei concetti cardine del pensiero shaftesburiano: la spontanea inclinazione verso gli altri uomini, ossia l'impulso alla socialità, e la politeness. Come le norme di comportamento del polite Gentleman proibiscono di sottrarsi alla conversazione in società, tanto più il divieto è valido nel caso di una conversazione con se stessi: la forma primaria e fonte originaria dell'impulso alla socialità, da cui derivano anche le regole della politness, si manifesta dunque proprio nella solitudine.

In questo contesto Shaftesbury offre una nuova interpretazione del motto Gnothi seauton, "conosci te stesso" per spiegare la possibilità del dialogo interiore: "Questa era tra gli antichi la celebre iscrizione delfica "conosci te stesso", che equivaleva a dire "dividi te stesso" o "sii due persone" ${ }^{22}$. Il soggetto in altre parole si divide, si raddoppia e diviene oggetto di se stesso:

Chiunque fosse, anche per poco, un attento esaminatore, finirebbe inevitabilmente col conoscere il suo cuore. $\mathrm{E}$ - cosa singolare di questi specchi magici - accadeva che, attraverso un lungo e costante esame, le persone acquisivano un abito speculativo peculiare, come se di fatto portassero con sé una sorta di specchietto tascabile, sempre a portata di mano e pronto all'uso ${ }^{23}$.

Il cammino verso la conoscenza, dunque, è fin dall'inizio legato e condizionato dalla dimensione dialogico-sociale. Il

22. Ibid: "This was, among the ancients, that celebrated Delphic inscription, "Recognize yourself", which was as much as to say "Divide yourself!" or "Be two!"»; trad. it. p. 215.

23. Ivi, p. 87: «No one, who was ever so little a while an inspector, could fail of becoming acquainted with his own heart. And - what was of singular note in these magical glasses - it would happen that, by constant and long inspections, the parties accustomed to the practice would acquire a peculiar speculative habit, so as virtually to carry about with them a sort of pocket-mirror, always ready and in use»; trad. it. p. 229. 
Metamorfosi dei Lumi 10. L'emergenza del sentimento

dialogo diviene lo specchio in cui il soggetto letteralmente si riflette, ovvero riflette su se stesso e sfugge alla «filosofia iper-speculativa» (super-speculative philosophy) per passare a «un genere più pratico, che concerne principalmente la nostra familiarità, amicizia e buona comunicazione con noi stessi» ${ }^{24}$. Infine il dialogo si trasforma poi in strumento di una riflessione condivisa fra più soggetti all'interno della società. Il passaggio dal solipsismo alla conversazione è una conditio sine qua non del pensare e dell'ampliamento del sapere.

Cercheremo ora di mostrare come la concezione antropologica e morale di Shaftesbury e il percorso che lo porta dalla teoria delle affezioni all'ideale di una solitudine sociale all'interno della quale i pensatori elaborano e condividono il sapere si riflettano e vengano rielaborati nelle opere del medico e filosofo svizzero Johann Georg Zimmermann.

\section{Zimmermann e la riflessione antropologica sulla solitudine} Zimmermann (1728-1795) compie i suoi studi all'università di Gottinga, dove è allievo di Haller. Dopo diversi anni in giro per l'Europa, viene chiamato come medico di corte da Giorgio III, re di Inghilterra e principe elettore di Hannover. In seguito sarà anche al capezzale dell'imperatore Federico II, alla cui figura dedicherà numerosi scritti.

La fama di Zimmermann è però legata principalmente a quattro opere: Von der Erfahrung in der Arzneiwissenschaft (Sull'esperienza in medicina) del 1764, che discute il concetto di esperienza e osservazione empirica in relazione ai pregiudizi e alla capacità di induzione del medico, e i tre libri sulla solitudine: Betrachtungen über die Einsamkeit (Considerazioni sulla solitudine) del 1756, Von der Einsamkeit (Della solitudine) del 1773 e Über die Einsamkeit (Sulla solitudine) del 1784-1785.

I titoli di queste tre opere mostrano una chiara evoluzione di modalità e intenti: il primo testo è concepito come una raccolta di considerazioni di carattere morale, filosofico e religioso presentate in forma poco più che aforistica e con un linguaggio spesso poetico e metaforico; in esso Zimmermann per lo più riflette sulla propria esperienza di solitudine sulle 
Coltivare le emozioni tra solitudine e socialità. Gli esempi di Shaftesbury e Zimmermann

Alpi bernesi, tanto che Bodmer, in una lettera del 4 ottobre 1756, gli suggerisce di sostituire in a über trasformando dunque il titolo in Considerazioni in solitudine ${ }^{25}$.

Il secondo testo, Von der Einsamkeit, è già più sistematico e contiene due dei nuclei che saranno fondamentali nell'ultimo saggio, ovvero la ricerca degli impulsi naturali che spingono alternativamente alla socialità o alla solitudine, e che sono chiaramente un'eco della teoria shaftesburiana, e la critica dell'ascetismo monastico delle origini, le cui argomentazioni verranno però poi rivolte anche contro il pietismo.

Infine abbiamo Über die Einsamkeit, opera monumentale in quattro volumi per un insieme di più di duemila pagine. Il primo capitolo muove da una serie di precisazioni sul concetto di solitudine su cui è opportuno soffermarsi:

Solitudine è una condizione dell'anima in cui quest'ultima si abbandona alle proprie riflessioni. Godendo di reale isolamento e di grande quiete o anche solo distogliendo il pensiero da ciò che ci circonda, noi siamo soli ${ }^{26}$.

Questa definizione include al suo interno due forme di solitudine in realtà piuttosto diverse; da un lato vi è la solitudine spaziale, in cui l'individuo si trova fisicamente in un luogo appartato, lontano dai suoi simili, ma conserva ancora il contatto con il mondo naturale: è il caso, per esempio, dell'esperienza fatta dallo stesso Zimmermann sulle Alpi bernesi o, ancora, quella dei pastori, che non a caso egli cita poche righe dopo; dall'altro, vi è la solitudine interiore, in cui l'uomo trascende non solo la dimensione sociale, ma anche la realtà sensoriale: è il caso, per esempio, dei monaci di clausura, ma anche dei dotti che trascorrono la vita nei loro studi. Va subito precisato che, fra le due forme, la preferenza di Zimmermann va nettamente alla prima.

Che vi siano diversi tipi di solitudine emergeva già in un passo delle Betrachtungen dove, non a caso, compare per la prima volta il nome di Shaftesbury:

25. Lettera di Bodmer a Zimmermann del 4 ottobre 1756 in E. Bodemann, Johann Georg Zimmermann. Sein Leben und bisher ungedruckte Briefe, Hahn, Hannover 1878, p. 164.

26. J.G. Zimmermann, Über die Einsamkeit, 4 voll., vol. I, p. 3: «Einsamkeit ist eine Lage der Seele, in der sie sich ihren eigenen Vorstellungen überläßt. In Genusse wirklicher Absonderung und großer Stille, oder auch nur durch Wegwendung der Gedanken von dem, was uns umgiebt, sind wir einsam». 
Metamorfosi dei

Lumi 10.

L'emergenza

del

sentimento

Nessun monaco solitario, dice Shaftesbury, nessun eremita, è mai stato veramente da solo: unicamente il saggio è in grado di trarre vero e autentico giovamento dalla lontananza dal mondo, e quanto rari sono i saggi? ${ }^{27}$

Questa citazione dal Soliloquio coglie appieno la differenza tra "solitudine sociale" e mero solipsismo delineata da Shaftesbury e la esplica facendo apertamente coincidere il solipsismo con una forma inautentica di solitudine. Quella autentica, invece, è riservata al saggio, il solo a poter trarre giovamento dal suo allontanarsi dal mondo in quanto capace, nella solitudine, di osservare se stesso. Il nosce te ipsum era già al centro di un appunto di diario del $1753^{28}$ e diventa il nucleo di un testo dal titolo Von der Diät für die Seele (Della dieta per l'anima) del 1764 in cui alle res non naturales della dietetica per il corpo Zimmermann ne sostituisce altre riferite all'anima ${ }^{29}$. La figura del saggio scivola così progressivamente in quella del medico, che dopo aver analizzato se stesso è in grado di applicare il proprio sapere agli altri.

È stato sostenuto che in questo ampliamento del discorso al campo medico Zimmermann si staccherebbe da Shaftesbu$\mathrm{ry}^{30}$, ma in realtà concetti quali dietetica e terapia erano ben presenti nel discorso shaftesburiano, tanto che negli Askemata la filosofia viene fatta coincidere con l'atto di cura. E l'immagine citata dell'uomo che dapprima è paziente e poi medico di se stesso ne è una conferma. Si può semmai sostenere che Shaftesbury non è interessato alla dimensione professionale dell'attività curativa, in quanto egli è certo un precursore, ma ancora non incarna la figura del medico-filosofo come farà invece Zimmermann.

27. J.G. Zimmermann, Betrachtungen über die Einsamkeit, Heidegger und Compagnie, Zürich 1756, p. 15: «Kein abgesonderter Mönch, sagt Shaftesbury, kein Eremit ist jemahls wahrhaftig allein gewesen - den wahren und eigentlich nutzen aus der Entfernung zu ziehen, ist nur der Weise im Stande, und wie selten ist ein Weiser?».

28. J.G. Zimmermann, Nosce te ipsum. Zimmermanns Tagebuch aus dem Jahre 1753, a cura di C. Hummel, Brugger Neujahrsblätter Nr. 85, Brugg 1975.

29. Cfr. C. Frey, Laune: Poetiken der Selbstsorge von Montaigne bis Tieck, Fink, München 2017, p. 26.

30. H.-G. Dehrmann, Produktive Einsamkeit: Gottfried Arnold, Shaftesbury, Johann Georg Zimmermann, Jacob Hermann Obereit, Christoph Martin Wieland, Wehrhahn, Hannover 2002, p. 84. 
Coltivare le emozioni tra solitudine e socialità. Gli esempi di Shaftesbury e Zimmermann
Il vero elemento innovativo del pensiero di quest'ultimo consiste semmai nel fatto che la forma autentica di solitudine da lui postulata prevede si preservi il contatto con la natura poiché essa dà impulso alla fantasia e permette un pieno sviluppo dei sensi. Se per Shaftesbury la natura era poco più di un mero fondale, era lo spazio in cui l'isolamento si realizza con l'obiettivo di mantenere in equilibrio le affezioni, mentre scarso interesse era rivolto alle facoltà inferiori dell'anima, Zimmermann, da profondo interprete qual è della coeva discussione antropologica sul ganzen Mensch ${ }^{31}$, sull'uomo nella sua complessa e poliedrica totalità, mira a uno sviluppo armonioso dell'intelletto, della sensibilità e dei sensi. 
Metamorfosi dei

Lumi 10.

L'emergenza del sentimento
Strong emotions of terror: emozione e politica in Edmund Burke

Lorenzo Rustighi

Trattando del tema dell'emozione nel pensiero di Edmund Burke, si cercherà di mostrare in che modo esso sia profondamente legato al suo contributo filosofico-politico. Lo si farà in particolare confrontando due delle sue opere più note, molto distanti nel tempo e apparentemente prive di relazione l'una con l'altra: A Philosophical Enquiry into the Origin of our Ideas of the Sublime and Beautiful, pubblicata nel $1757 \mathrm{e}$ in una seconda edizione nel 1759, e le Reflections on the Revolution in France, risalente al 1790. La prima è un'opera che potremmo definire di teoria estetica, dove le passioni e le emozioni occupano comprensibilmente un posto centrale. Il secondo è un testo di intervento politico, legato ai recenti fatti rivoluzionari francesi e redatto da Burke in risposta al sermone tenuto dal pastore non conformista Richard Price presso la Revolution Society il 4 Novembre del 1789. L'obiettivo primario di questa rassegna sarà quello di esplorare uno dei punti più controversi nella letteratura dedicata a Burke, vale a dire il rapporto che intercorre tra la sua articolazione delle categorie estetologiche di bello e sublime e il modo in cui il parlamentare inglese pensa le strutture politico-costituzionali. Gli interpreti hanno ampiamente notato, infatti, come i concetti elaborati da Burke in ambito costituzionale - nelle 
Strong emotions of terror: emozione e politica in Edmund Burke
Riflessioni, ma anche nei significativi interventi dedicati alle colonie orientali, all'Irlanda e all'indipendenza americana - risentano profondamente delle chiavi di lettura da lui adottate in sede di analisi estetica. La natura di questo nesso rimane tuttavia ancora poco chiara, nonostante molto sia stato scritto in proposito. Sbrogliare questa matassa di interpretazioni sarà utile, ci sembra, proprio a illuminare il ruolo dell'emozione politica nel pensiero burkiano.

\section{L'oggettività dell'emozione}

L'Inchiesta sul bello e il sublime si inserisce, come noto, in un più vasto panorama di riflessioni sul tema del sublime che si erano moltiplicate sin dalla seconda metà del secolo precedente sulla scorta di alcune traduzioni del celebre Peri Hýpsous dello Pseudo-Longino, probabilmente databile al I secolo d.C. La prima traduzione inglese risale al 1652 a opera di John Hall, ma la vera fortuna, anche in Inghilterra, si deve alla traduzione francese di Boileau del 1674, tradotta poi in inglese nel 1680 da John Pulteney ${ }^{1}$. Tradizionalmente il sublime non era altro che uno degli stili definiti dalla retorica antica: era quello stile grande, magnifico e terribile adatto a temi di una certa gravità, come gli argomenti eroici. A partire dalla seconda metà del Seicento il sublime, già con Boileau, inizia a diventare qualche cosa di diverso da una questione soltanto stilistica, perché da un lato viene progressivamente identificato con l'effetto emotivo prodotto, indipendentemente dal registro utilizzato, e dall'altro, di conseguenza, l'attenzione si sposta sempre di più dal poeta al lettore. Il classicismo sei e settecentesco produce quindi una vasta serie di considerazioni e rielaborazioni sul tema che non sarebbe possibile qui affrontare. Basti ricordare il contributo fondamentale dato da autori come Shaftesbury, Dennis e soprattutto Addison, che come Burke reinterpreta Longino a partire dall'empirismo di impronta lockiana. A segnare la rottura di Burke con Addison è tuttavia il fatto che per quest'ultimo sussiste ancora una netta separazione tra patetico e sublime, come era già per Longino, subordinando così il pathos all'immaginazione: Burke, al contrario, non solo riconnette i due momenti, ma

1. Si veda in proposito l'esaustiva introduzione di Sertoli all'Inchiesta: G. Sertoli, Presentazione, in E. Burke, Inchiesta sul bello e il sublime, a cura di G. Sertoli e G. Miglietta, Aesthetica edizioni, Palermo 1985, pp. 9-40. 
Metamorfosi

dei

Lumi 10.

L'emergenza

del

sentimento soprattutto tende progressivamente a dare all'immaginazione un ruolo di secondo piano, e questo come vedremo sarà un aspetto decisivo della sua dottrina tanto estetica quanto politico-morale.

Per introdurre il problema che qui ci occupa, quello dell'emozione, sembra doveroso cominciare dalla definizione che lo stesso Burke ne fornisce nell' Inchiesta. Si tratta per lui di una questione eminentemente fisiologica, dove esiste una strettissima connessione tra la mente e il corpo. A proposito delle passioni dolorose, di cui tratteremo tra poco, Burke scrive: «Il dolore e il timore agiscono sulle stesse parti del corpo e nel medesimo modo, sebbene differiscano in intensità; il dolore e il timore consistono in una tensione anormale dei nervi, accompagnata talvolta da una forza anormale che a volte improvvisamente si tramuta in una straordinaria debolezza» $»^{2}$. E prosegue: «L'unica differenza fra il dolore e il timore è che le cose che producono dolore agiscono sulla mente mediante l'intervento del corpo, mentre le cose che producono timore di solito colpiscono gli organi del corpo per mezzo della mente, ma entrambi, accordandosi subito o in un secondo tempo nel produrre una tensione, contrazione, o violenta emozione dei nervi, si accordano ugualmente in tutto il resto» ${ }^{3}$. Tra eccitazione fisiologica e eccitazione dell'anima vi è dunque un necessario accordo, tanto che sarebbe difficile, secondo Burke, stabilire una netta gerarchia. Il che lo induce a rinunciare a una qualche teoria compiuta del reciproco implicarsi di mente e corpo, teoria che sarebbe a suo avviso inevitabilmente confusa. Ciò che è interessante a proposito del sublime però è questo: «Dopo aver considerato il terrore come la causa di una tensione anormale e di violente emozioni dei nervi, segue facilmente che tutto ciò che è atto a produrre una tale tensione, deve produrre una passione simile al terrore, e per conseguenza deve essere una fonte di sublime, pur non essendo connesso con alcuna idea di pericolo» ${ }^{4}$. Tutte le spiegazioni che possiamo dare, per Burke, si fermano a questo livello: c'è qualcosa che è adatto a produrre una determinata emozione agendo o sulla mente

2. E. Burke, Inchiesta, cit., p. 143.

3. Ivi, pp. 143-144.

4. Ivi, p. 145 . 
Strong emotions of terror: emozione e politica in Edmund Burke o sul corpo, ma il cui effetto resta indipendente da entrambi ed è quindi in qualche modo oggettivo.

Ciò che interessa a Burke infatti è isolare alcuni principi fondamentali della sensibilità umana in grado di rendere ragione delle nostre passioni. Come è noto, ne individua essenzialmente due, che sono in definitiva quelli tradizionali: piacere e dolore, che corrisponderanno ai due assi fondamentali della sua estetica, il Bello e il Sublime. Ora, ciò che gli preme è evitare ogni tipo di soggettivismo nell'esaminare le passioni. Non si tratta per lui di principi suscettibili di variazioni che non siano puramente fisiologiche, dipendenti dalla diversa costituzione di ciascuno di noi. Per il resto, l'emozione è qualcosa di universale su cui gli uomini possono convenire, al di là di ogni scetticismo - come lo chiama Burke - che pretenda di revocare in dubbio «l'accordo delle nostre percezioni $»^{5}$. La nozione di agreement, accordo, è centrale in questo senso: «Si deve necessariamente riconoscere che i piaceri e i dolori che ogni oggetto eccita in un uomo deve suscitarli in tutta l'umanità, quando agisca in forma naturale, semplice, e solo in base ai suoi precisi poteri» ${ }^{6}$. Gli uomini dunque concordano sostanzialmente sui principi del gusto come su quelli del piacere e del dolore. Si tratta di una concezione non soggettivistica proprio perché per Burke ciò che conta qui (ma, come vedremo, anche nella sfera politica) non è la volontà, la scelta, la personale inclinazione, ma una necessità cogente. La contraddizione, il reciproco escludersi di volontà e necessità, deve dunque costituire il filo rosso della sua intera indagine.

\section{La natura del bello e del sublime}

Come si definiscono allora i due principi menzionati, quelli del piacere e del dolore, del bello e del sublime? Abbiamo a che fare con «idee semplici, non suscettibili di definizione» ${ }^{7}$, proprio perché si tratta di esperienze su cui esiste un sostanziale accordo. Nel caso del piacere, potremmo dire che ha a che fare con la vita e con la sua riproduzione. Il dolore, invece, è legato alla morte o quantomeno ne evoca il pericolo. Per Burke si tratta tuttavia di due principi radicalmente
5. Ivi, p. 51.
6. Ibid.
7. Ivi, p. 66. 
Metamorfosi

dei

Lumi 10.

L'emergenza

del

sentimento

130 indipendenti l'uno dall'altro, che non possono essere pensati come due gradi diversi della stessa esperienza. Piacere e dolore sono passioni positive, che non si definiscono per privazione o per eccesso - il piacere non è la scomparsa o la diminuzione del dolore - ma per una pura presenza. Tra le due non v'è quindi passaggio graduale, ma uno stato di quiete che Burke chiama «indifferenza». Secondo Burke, per esempio, quella sofferenza determinata dalla perdita dell'amore non è un reale dolore, proprio perché assume significato solo a partire dal piacere di cui è negazione. Lo stesso vale per ciò che chiama delight, il diletto, che non è una forma di piacere ma si produce solo allorché un dolore diminuisca o più semplicemente quando esso si trovi a una certa distanza da noi, senza riguardarci realmente. Burke parla a questo proposito di delightful horror, che è in qualche modo la cifra dell'esperienza del sublime quale è considerata ancora oggi nel linguaggio comune. Ora, tra le due passioni del piacere e del dolore Burke non ha dubbi: la seconda è certamente quella più forte, più vivida. È quella che produce strong emotions of horror, espressione che nel titolo di questo contributo è stata modificata in riferimento al terrore rivoluzionario di cui tratteremo tra poco. Nessun piacere per Burke potrà mai superare in intensità lo smarrimento generato dalla sofferenza, dalla paura o dal pericolo di morte.

È a partire da qui che Burke può definire le due modalità estetiche della bellezza e della sublimità. Anche in questo caso si tratta di due esperienze del tutto contrarie, tanto è vero che Burke le associa in maniera piuttosto esplicita rispettivamente alla femminilità e alla mascolinità, segnate a suo avviso da una differenza irriducibile. Il femminile, o meglio ancora il materno, sarebbe il luogo del bello, mentre il maschile e il paterno quello del sublime. Un aspetto curioso, di cui si renderà ragione più tardi, è che il dilettevole sia associato da Burke non tanto alla figura del padre, che incute timore e reverenza, ma a quella del nonno, che ha un'autorità simile a quella paterna e tuttavia più distante, meno imminente, quindi capace di produrre una forma di godimento. Ora, Burke impiega molte pagine per spiegare che cosa il bello e il sublime non siano, prima di esporne le qualità positive. Soprattutto per quanto riguarda il bello, occorre a suo avviso sgomberare il campo da tutta una serie di errori che tendono ad associare la bellezza alla proporzione, alla perfezione e alla maestosità. Al contra- 
Strong emotions of terror: emozione e politica in Edmund Burke rio, bello non è ciò che è proporzionato, nel senso che non è questo che fa del bello ciò che è. Una cosa bella può essere sproporzionata, tanto quanto può essere imperfetta. Meglio ancora, il bello deve essere imperfetto, deve comunicare un sentimento di precarietà e di debolezza. Qui è ancora l'idea di femminilità a giocare un ruolo decisivo, perché la bellezza femminile per Burke non può essere dissociata dalla sua fragilità. E così una cosa bella non deve essere grande e magnifica, ma al contrario deve essere piccola, minuta. Questo anche a motivo del fatto che la bellezza si associa secondo Burke alla chiarezza, e ciò che è troppo grande non può essere immediatamente compreso dallo sguardo e dall'intelligenza: «Un'idea chiara è quindi un altro modo di dire un'idea piccola» ${ }^{8}$. Il contrario vale per il sublime. Le cose sublimi sono per essenza grandi, immense, anzi infinite; sono poi confuse, non ben distinte, sono difficili da cogliere e da comprendere; infine sono legate all'insicurezza della notte e della scarsa visione. Se quindi la bellezza è sinonimo di luminosità il sublime è invece sinonimo di oscurità.

Tutto questo spiega anche le due forme di relazione che si fondano sulle due modalità dell'esperienza estetica: il bello genera amore, anche in un senso propriamente carnale, mentre il sublime produce ammirazione, reverenza, sottomissione. Da cui segue, secondo Burke, che mentre le cose belle sono tali perché ci spingono a conquistarle, ad assoggettarle, le cose sublimi ci inducono invece a sottometterci a esse. L'oggetto d'amore è qualcosa che vogliamo possedere, l'oggetto della reverenza è ciò a cui tendiamo a obbedire. La sfera del sublime è infatti la sfera del comando e più in generale del governo. In quanto tale è qualcosa di intrinsecamente terribile, che suscita nel nostro animo la paura di essere sopraffatti e addirittura distrutti. Il tratto distintivo del sublime è infatti ciò che Burke chiama astonishment, che curiosamente associa a un'altra passione fondamentale di cui vedremo il carattere propriamente politico, cioè la vergogna. Burke collega il sublime all'area semantica del verbo latino vereor, che ha che fare proprio con il pudore ma anche con il rispetto e l'ammirazione. Qui riemerge la questione della sottomissione alla necessità, dovuta all'annullamento della volontà soggettiva

8. Ivi, p. 90. 
Metamorfosi dei

Lumi 10.

L'emergenza

del

sentimento

132 e perfino della razionalità come effetto dell'attonimento. $\mathrm{Si}$ tratta di un aspetto decisivo del sublime burkiano, che nella Critica del Giudizio Kant cercherà invece di superare. Il sublime è allora in senso lato il regno della potenza, Power, come è titolato il capitolo V della seconda sezione dell'Inchiesta. Burke è esplicito da questo punto di vista: «Il potere che in base a una legge costituita detengono i re e i comandanti ha la stessa connessione col terrore. I sovrani sono spesso designati col titolo di terribile maestà ${ }^{9}$. Lo stesso si può certamente dire di Dio, anche se con una distinzione fondamentale: altra cosa è produrre un'idea il più possibile chiara e distinta di Dio e dei suoi attributi, facendone dunque una teologia, altra cosa invece è tentare di rappresentarsi la potenza divina in tutta la sua trascendenza. Nel primo caso non c'è terrore, c'è al contrario piacere intellettuale; nel secondo invece ci sentiamo schiacciati dalla sua grandezza. Una considerazione analoga a proposito del concetto di power si trova nel capitolo dedicato al concetto di oscurità, che come abbiamo visto è un carattere proprio del sublime: «Quei governi dispotici che si basano sulle passioni degli uomini, e principalmente sulla paura, sottraggono il più possibile i loro capi alla vista della moltitudine» ${ }^{10}$. La potenza è di per sé inattingibile, difficile da mettere a fuoco, e questo vale in particolare per la tirannide, che si rende massimamente invisibile, distante, celata nel mistero.

\section{Passioni politiche}

È chiaro quindi come bello e sublime ci proiettino in una dimensione di tipo politico: volontà e libertà, da un lato, comando e obbedienza, dall'altro. Si tratta di due ambiti radicalmente contrari, come si è detto, di cui occorrerà tuttavia comprendere l'effettivo rapporto - o, meglio ancora, l'unità - nella dimensione costituzionale burkiana.

Non è solo in questa direzione, però, che può essere sviluppata la politicità delle due categorie. Il sublime infatti è anche da associarsi, secondo Burke, al nostro naturale impulso a fuggire la morte e a mantenerci in vita. Il bello è invece connesso a passioni più propriamente sociali, che si distinguono in due categorie: le passioni legate alla società

9. Ivi, p. 93.

10. Ivi, p. 86 . 
Strong emotions of terror: emozione e politica in Edmund Burke dei sessi e quelle legate alla società in generale. Nel primo caso si parla di passione erotica in senso stretto, che è il dominio più proprio della bellezza. Ne consegue che quella coniugale sia la forma più semplice di società. L'amore è infatti propriamente la passione della generazione, della riproduzione di se stessi e dei propri legami. Nel caso della società in generale, invece, Burke distingue tre passioni assai più ambigue, perché al contrario dell'amore non sono immediatamente riconducibili al bello nella sua purezza ma manifestano alcuni elementi di sublimità. Questo è particolarmente vero per la prima, la simpatia. Un concetto che Burke ricava evidentemente dall'empirismo e in particolare da Hume, con cui ha rapporti diretti, e che è qualcosa di molto simile a ciò che in Francia Rousseau aveva definito pitié, la pietà: la simpatia è infatti la capacità di provare le stesse passioni che provano gli altri, ma è dettata non tanto da un meccanismo al tempo stesso fisico e morale di comunicazione, quanto da una dinamica di vera e propria sostituzione. È un processo di natura «metaforica» per Burke, in base al quale non soltanto siamo in grado di metterci al posto di qualcun altro ma più in generale di produrre effetti di sostituzione linguistica che stanno alla base di ciò che per Burke è la vera essenza delle passioni estetiche. La seconda passione sociale è opposta a quest'ultima, anche se apparentemente molto simile, ed è quella dell'imitazione: passione per così dire riproduttiva e quindi più affine all'amore e al piacere che al dolore, perché ci spinge a imitare gli altri e a ripetere i loro comportamenti. Infine, la terza passione sociale, che tempera quella dell'imitazione, è l'ambizione, che ci induce a distinguerci, a introdurre qualcosa di nuovo nel processo imitativo, e dunque chiama in causa l'orgoglio, l'onore, il coraggio. È chiaro che per Burke queste tre passioni non si escludono, ma anzi coesistono, così come coesistono l'amore sessuale e l'autopreservazione. Occorre mettere l'accento proprio su questa compresenza di elementi molto diversi, se non opposti, che è uno dei tratti propri del pensiero politico di Burke ${ }^{11}$ e che ha a che fare di nuovo con una dimensione di necessità e di oggettività. 
Metamorfosi dei

Lumi 10.

L'emergenza

del

sentimento

134
A questo punto si affaccia però una difficoltà sostanziale. Se la vera causa del sublime deve essere ricercata nel rischio della morte, allora esso sembra appartenere a un ambito strettamente individuale, quello della self-preservation appunto, e non a una dimensione politica quale in prima battuta sembrava essere quella dell'autorità e del comando. In effetti, come si è visto, Burke distingue nettamente le passioni legate all'autopreservazione da quelle che concernono la società. Viceversa, sarebbero proprio i sentimenti legati alla sfera del piacere e in particolare il sentimento dell'amore - proprio perché ha a che fare con l'unione e in primo luogo con la famiglia - a promuovere non tanto la libertà soggettiva $\mathrm{e}$ la volontà individuale, che sono fattori di separazione, ma piuttosto la relazione tra gli uomini e il superamento di ogni possibile indipendenza. In definitiva, le due dimensioni estetiche burkiane sembrano ora dover presupporre ciò che a una prima considerazione senz'altro escludevano.

Una prima soluzione potrebbe essere fornita dalla constatazione, da parte di Burke, che stare in società non è di per sé un vero e proprio piacere, un'esperienza di godimento che ricerchiamo per se stessa, ma ha a che fare con il dolore causato dalla solitudine, che è qualcosa di molto vicino alla morte e che dunque siamo spinti a evitare. Potremmo dire allora che se la socialità non è in sé piacevole è tuttavia dilettevole, proprio perché dipende dalla possibilità di guardare da lontano il dolore del perfetto isolamento. In questo senso anche la relazione sociale rientrerebbe a tutti gli effetti in una tonalità emotiva che rientra nel quadro dell'esperienza sublime. Non è qui, tuttavia, che Burke ci fornisce una risposta adeguata alla contraddizione che sembra minacciare la sussistenza del bello e del sublime come dimensioni autonome e reciprocamente non contaminate. Ciò che occorre fare, piuttosto, è approfondire questa contraddizione e comprenderne il significato per così dire dialettico all'interno del pensiero di Burke. In questo senso è utile seguire l'interpretazione proposta da Thomas Mitchell, che resta un riferimento importante negli studi burkiani e che fa notare precisamente come il bello e il sublime subiscano in Burke 
Strong emotions of terror: emozione e politica in Edmund Burke un costante movimento di rovesciamento l'uno nell'altro: «[It] might be called the principle of dialectical reversal, a process in which oppositions seem to change places. Burke sees this at times as something like a natural law, a coincidentia oppositorum» ${ }^{12}$.

Per comprendere questo punto, è necessario fare cenno alla lettura che Mitchell offre dell'estetica burkiana. In prima battuta, egli nota, il sublime in Burke sembra essere inseparabile dall'immaginazione: proprio perché ha a che fare con l'informe, con l'oscuro, con l'infinito, le passioni che esso suscita agiscono sull'immaginazione, come nel caso della paura dei pericoli che la notte nasconde. E tuttavia Mitchell sottolinea anche come nelle sezioni conclusive dell' Inchiesta Burke svincoli radicalmente il sublime dall'immagine, dall'idea e, cosa rilevante, dall'imitazione. Si potrebbe dire che Burke ci porti alla conclusione che laddove ci siano immagini sensibili ci si muova solo nell'ambito del bello, non in quello del sublime. I due esempi che meglio chiariscono questa distinzione sono quelli della pittura e della poesia. Un sublime pittorico è un sublime improprio: un'immagine difficilmente desta reale terrore e smarrimento, non importa quanto l'oggetto rappresentato sia terribile, proprio perché è un'imitazione di qualcosa e dunque per così dire è interamente disponibile alla nostra capacità di farcene un'idea, di possederlo nel pensiero. Davvero sublime può essere soltanto la poesia, ma di nuovo, non perché sia in grado di rappresentare meglio della pittura qualcosa di terrificante, bensì proprio perché la poesia non rappresenta alcunché: il suo potere sta nelle possibilità di combinazione semantica disponibili al linguaggio, indipendentemente dai significati che le parole veicolano. Per questo «la poesia, nel suo senso più generale, non può essere chiamata in senso stretto un'arte di imitazione [...]. La poesia descrittiva opera soprattutto per sostituzione, per mezzo di suoni che per abitudine producono l'effetto di realtà. Non v'è imitazione al di fuori della somiglianza e le parole indubbiamente non hanno rassomiglianza con l'idea a cui si riferiscono» ${ }^{13}$. Per questo Burke sostiene non solo che è

12. T. W. J. Mitchell, Eye and Ear: Edmund Burke and the Politics of Sensibility, in Id., Iconology: Image, Text, Ideology, The University of Chicago Press, Chicago 1987, pp. 116-149, qui p. 128.

13. E. Burke, Inchiesta, cit., p. 175. 
Metamorfosi

dei

Lumi 10.

L'emergenza

del

sentimento

136 possibile che le parole generino una violenta passione in noi senza essere accompagnate da alcuna rappresentazione corrispondente, ma addirittura che tutto il linguaggio funziona così. Per parlare non abbiamo bisogno di idee, di immagini mentali. Queste sono qualcosa di derivato, frutto di un'astrazione successiva del pensiero: «La mente possiede la facoltà di suscitare immagini a piacere; ma allora è necessario per questo un atto di volontà, e nella ordinaria conversazione o lettura accade molto di rado che un'immagine sorga nella mente» ${ }^{14}$. Ancora una volta sembra esserci una netta distinzione tra l'atto della volontà e la necessità inerente ai meccanismi del linguaggio. Come Mitchell osserva, l'operazione di sostituzione di cui parla Burke è quella della simpatia, e come tale non richiede una postura di tipo imitativo ma piuttosto l'abitudine, la consuetudine, l'educazione. Dobbiamo intendere questi termini in un senso empirista, nel senso delle impressioni che l'esperienza ha lasciato su di noi e che ci portano a sostituire abitualmente alle parole che ascoltiamo una sequenza di tracce sedimentate, senza che ci sia alcuna operazione rappresentativa. Insomma, parlare è qualcosa di involontario, dentro la cui necessità impersonale siamo presi, e si fonda su quell'accordo della sensibilità di cui Burke parla all'inizio del suo trattato.

A questo livello però l'interpretazione di Mitchell mostra un limite fondamentale, perché tende a riprodurre la dicotomia tra bello e sublime di cui riconosceva tuttavia la debolezza. Osserva infatti che, se il bello ha a che fare con la vista e con l'occhio, il sublime è invece legato all'ascolto e all'orecchio. Questa a suo avviso è anche la differenza che nelle Riflessioni del 1790 rende ragione dello iato tra lo spirito rivoluzionario francese, che è spirito della chiarezza teorica, della libertà, della volontà e quindi della bellezza, e lo spirito costituzionale inglese, che è spirito sublime perché legato alla struttura simpatetica del linguaggio, al costume e alla necessità. Questa lettura porta Mitchell a sostenere che vi sarebbero in Burke un sublime autentico, incarnato proprio in Inghilterra, e un sublime contraffatto, che si manifesterebbe proprio nello spettacolo rivoluzionario francese e che sarebbe di fatto illegittimamente contaminato dalla dimen- 
Strong emotions of terror: emozione e politica in Edmund Burke sione del bello. Questa linea interpretativa ha avuto molto successo $^{15}$, ma non si dimostra fino in fondo coerente con l'impostazione burkiana: se è vero che il bello e il sublime non possono essere effettivamente divisi, pena il loro infinito ricadere l'uno nel proprio opposto, come sarebbe possibile concepire un sublime puro e intatto - quello della buona costituzione - che non faccia i conti con ciò che è altro da sé, con la bellezza?

\section{Il sublime rivoluzionario}

A questo punto è possibile esaminare alcune delle questioni più rilevanti che emergono nelle Riflessioni sulla Rivoluzione in Francia. Per prima cosa, è il caso di notare che si tratta di un testo composto interamente all'insegna della cifra emotiva, per non dire patetica. Ancora Mitchell parla non a caso, a proposito di questo e altri scritti burkiani, di una «politica della sensibilità», mentre Furniss e Reid la definiscono una «politica del gusto» ${ }^{16}$. Ł̀ un testo redatto in forma epistolare, indirizzato probabilmente a Charles-Jean-François Depont, consigliere del Parlamento di Parigi, e prende le mosse dalla feroce critica che Burke muove alla cosiddetta Revolution Society, fondata attorno alla metà del XviII secolo per la commemorazione della Glorious Revolution inglese del 1688-1689. La Società aveva infatti preso le difese della Rivoluzione Francese e ne promuoveva i principi di fondo, specie attraverso la figura di Richard Price. In questo lungo testo Burke contesta punto per punto le tesi rivoluzionarie, in particolare quelle che sono riconducibili alle idee contrattualiste di Hobbes e Rousseau e più ancora di Sieyès, vero e proprio teorico del 1789. Lo fa, singolarmente, in una forma che può essere definita a pieno titolo sublime, perché mira a suscitare nel lettore passioni estremamente forti e violente. Questo è del

15. Cfr. S. White, Burke on Politics, Aesthetics, and the Dangers of Modernity, «Political Theory», 21 (1993), n. 3, pp. 507-527; T. Furniss, Edmund Burke's Aesthetic Ideology: Language, Gender, and Political Economy in Revolution, Cambridge University Press, Cambridge 1993; M. Neocleous, The Monstrous Multitude: Edmund Burke's Political Teratology, "Contemporary Political Theory», 3 (2004), n. 1, pp. 70-88; C. Carson, The King's Virtual Body: Image, Text, and Sovereignty in Edmund Burke's "Reflections on the Revolution in France», "Republics of Letters: A Journal for the Study of Knowledge, Politics, and the Arts», (2011), n. 2, pp. 115-126.

16. T. Furniss, Edmund Burke's Aesthetic Ideology, cit., pp. 68-88; C. Reid, The Politics of Taste, in D.E. Ritchie (a cura di), Edmund Burke: Appraisals and Applications, Transaction Publishers, New Brunswick 2016, pp. 61-78. 
Metamorfosi dei Lumi 10. L'emergenza del sentimento resto uno dei punti che gli furono rimproverati dai sui detrattori, a partire da Thomas Paine, che lo accusavano di procedere non per argomentazioni ma attraverso il puro effetto emotivo. Non solo infatti lo stile è particolarmente grave e magnifico, ma Burke indulge volentieri a passaggi di grande intensità allo scopo di comunicare orrore, raccapriccio, smarrimento. C'è in particolare una scena che è passata alla storia per l'enfasi e la crudezza della descrizione, cioè il resoconto dell'assalto alla residenza reale da parte di una moltitudine di rivoltosi la mattina del 6 ottobre 1789, dove Burke non risparmia tinte accese e immagini drammatiche: rappresenta Maria Antonietta svegliata di soprassalto e costretta a fuggire ancora seminuda, «una banda di scellerati assassini grondanti di sangue ${ }^{17}$ dopo aver fatto a pezzi una guardia, il palazzo «sommerso nel sangue, profanato dalle stragi, cosparso di membra e carcasse mutilate ${ }^{18}$, i soldati trascinati al ceppo e decapitati nel cortile, le loro teste infilate su picche trasportate durante la processione di ritorno a Parigi.

Ora, una domanda può essere legittimamente posta: perché proprio Burke, filosofo del sublime, ha visto nelle Rivoluzione francese una catastrofe epocale? Non fu forse l'evento più sublime dell'intero secolo? La sua avversione appare poi tanto più singolare se si pensa che Burke non solo era un fermo sostenitore dei principi della Glorious Revolution inglese ma aveva violentemente attaccato Warren Hastings per il suo malgoverno in India e preso le difese delle colonie americane contro la corona. A questa domanda è possibile rispondere, come si è anticipato, solo se si prende sul serio l'indicazione burkiana relativa all'impossibilità di separare i principi del bello e del sublime come due momenti perfettamente autosufficienti. Il vizio fondamentale della Rivoluzione francese, per Burke, consiste esattamente nella pretesa di operare una simile separazione. Il carattere più proprio del moto rivoluzionario in Francia e degli effetti che esso ha avuto sul piano della progettazione costituzionale non consiste infatti nel sublime ma piuttosto nel bello. La Rivoluzione si è fondata in altri termini sull'idea che fosse possibile mettere al centro della politica esclusivamente il piano della bellezza, che deve

17. E. Burke, Riflessioni sulla rivoluzione in Francia, in Id., Scritti politici, a cura di A. Martelloni, UTET, Torino 1963, pp. 149-443, qui p. 237.

18. Ivi, p. 238. 
Strong emotions of terror: emozione e politica in Edmund Burke

essere inteso in questo caso non solo come assolutizzazione di un momento genuinamente teorico, in cui un sistema di concetti chiari e distinti si rende principio ordinatore dell'intero disegno costituzionale - come Burke nota nel Ricorso dai nuovi agli antichi Whig, «una teoria concernente il governo può divenire una causa di fanatismo altrettanto efficacemente di un dogma religioso» ${ }^{19}$ - ma prima ancora come ipostatizzazione di ciò su cui quella teoria fa perno, cioè della semplice molteplicità empirica degli individui assunti come l'unica concreta particolarità e dunque come fondamento inalienabile dell'obbligazione politica. Sulla scorta delle teorie di tipo contrattualistico sorte nell'ambito del moderno diritto naturale a partire da Hobbes, la Rivoluzione ha preteso di porre la volontà collettiva dei cittadini come pietra angolare dello Stato, dando così assoluta rilevanza a un piano di pura immanenza, in apparenza perfettamente rappresentabile in un concetto della ragione perché empiricamente disponibile, e neutralizzando ogni possibile dimensione trascendente dell'autorità politica che a quella volontà non fosse interamente riconducibile. La Rivoluzione in altri termini, evento massimamente terribile e sublime, si è basata paradossalmente sull'esigenza di far fuori dall'orizzonte della politica ogni possibile elemento di sublimità. È proprio questa esigenza paradossale e contradditoria a segnarne il destino, che l'ha condotta per Burke precisamente a negare le proprie premesse istituendo un potere strutturalmente dispotico. Come Hegel noterà di lì a pochi anni, infatti, l'idea contrattualistica di rappresentare la volontà dello Stato come totalità delle volontà individuali, secondo il criterio dell'autorizzazione, si rovescia inesorabilmente nella necessità che questa volontà sia messa in forma da una volontà ulteriore, separata - quella dell'Assemblea Nazionale in questo caso - che trascende senza appello quel popolo di cui doveva essere semplice proiezione nelle istituzioni e di cui invece finisce per costituire la sola possibile manifestazione legittima ${ }^{20}$. Ne risulta, giocofor-

19. E. Burke, Ricorso dai nuovi agli antichi Whig, in Id., Scritti politici, cit., pp. 445579, qui p. 559.

20. Cfr. G.W.F. Hegel, Eticità assoluta e diritto positivo. Le maniere di trattare scientificamente il diritto naturale, trad. it. di M. Del Vecchio, FrancoAngeli, Milano 2011; G. Duso, La logica del potere. Storia concettuale come filosofia politica, Laterza, Roma-Bari 1999. 
Metamorfosi dei

Lumi 10.

L'emergenza

del

sentimento

140 za, un governo che proprio perché autorizzato ha i caratteri formali dell'assolutezza e dell'irresistibilità.

È proprio questo lo scenario cui Burke si trova a far fronte nelle Riflessioni. L'obiettivo primario è infatti quello di discutere una delle proposizioni centrali della Revolution Society, che stava sostenendo la Rivoluzione francese in Inghilterra con il plauso dell'Assemblea Nazionale: l'idea secondo la quale gli eventi francesi sarebbero in qualche misura l'equivalente di ciò che era stata la Glorious Revolution esattamente un secolo prima; un'idea fondata sul principio per cui un governo non è legittimo se non è stato scelto dal popolo e che dunque presuppone che la monarchia inglese, dopo il 1689, sia divenuta espressione della volontà popolare. Ora, l'intero testo di Burke è una critica serrata e progressiva di queste idee. Si tratta di dimostrare in primo luogo che tra la Glorious Revolution e la Rivoluzione francese non c'è alcuna affinità, ma una profonda opposizione. In secondo luogo, che il principio della legittimazione popolare conduce in realtà a conseguenze tiranniche. Qual è, anzitutto, la differenza sostanziale tra le due Rivoluzioni? La questione è semplice ai suoi occhi: la Glorious Revolution aveva avuto lo scopo di conservare e difendere la costituzione del regno, compromessa da un cattivo governo come quello di Giacomo I, laddove la Rivoluzione Francese ha un obiettivo contrario, quello di distruggere, polverizzare l'antica costituzione per costruirne da zero una completamente nuova proprio sulla base di una «teoria del governo». Burke impiega una metaforica di tipo medico di origine antica: in Inghilterra si è cercato di curare la parte malata, cioè la testa del corpo politico che è la monarchia, con il concorso delle parti ancora sane; in Francia invece si è voluto ridurre il corpo politico, «alle molecole organiche di un popolo frammentato in individualità» ${ }^{21}$. Qui riemerge quel conflitto tra necessità e volontà che abbiamo già osservato. In Inghilterra si è proceduto secondo necessità in un duplice senso: la necessità tragica di un atto rivoluzionario, che non è stato semplicemente «voluto» ma si è reso indispensabile, e la necessità dettata dall'ordine etico-politico di un assetto costituzionale di cui era necessario preservare la natura. In Francia invece ha prevalso la pura volontà astratta, 
fondata su ciò che Burke chiama polemicamente «i diritti dell'uomo», cioè sulle dottrine giusnaturaliste che alla $\mathrm{Ri}-$ voluzione hanno dato forma. Rimprovera Burke ai francesi: «Avete preferito agire come se non aveste mai conosciuto la società civile, come se doveste ricominciare tutto dai primi elementi» ${ }^{22}$, sulla base del presupposto che «una moltitudine contata per teste sia un popolo» ${ }^{23}$. Noi inglesi invece, afferma, «abbiamo preferito fare depositari dei nostri diritti e privilegi la natura e il cuore piuttosto che affidarci a speculazioni astratte e ritrovati ingegnosi» ${ }^{24}$.

Il punto decisivo risiede proprio nell'opposizione tra un campo di diritti plurali acquisiti, come quello inglese, e un campo del diritto declinato al singolare - «il vago diritto astratto» ${ }^{25}$, come lo chiama Burke. È infatti qui che emerge tutta la positività del sublime secondo Burke, un sublime incarnato proprio dalla costituzione inglese che conserva ancora intatti i principi delle costituzioni feudali. Ciò che la Glorious Revolution ha difeso non è infatti un diritto generico, ma i diritti e le libertà, i privilegi e le franchigie che il popolo inglese ha acquisito nel tempo attraverso i trattati, gli statuti, le consuetudini. È a questa altezza che deve essere compreso il concetto di «pregiudizio". Il pregiudizio è il contrario della nuda razionalità, di un intelletto preda della pura immaginazione e disconnesso dall'esperienza effettiva della vita in comune. È solo sulla base del pregiudizio, afferma Burke, che è possibile tornare a considerare la politica come facevano gli antichi, cioè non come scienza teoretica ma come scienza pratica, fondata dunque non sui principi immutabili dell'aritmetica ma sulla prudenza e sull'esperienza. Di nuovo allora una teoria del governo non può essere fatta, la politica non può essere ridotta interamente a quella forma minuta, chiara, compatta e levigata che è il delirio di bellezza di una ragione lasciata a se stessa. Per questo in Inghilterra, osserva, non si è lasciato che ciascuno fosse libero di condursi da sé, "sulla sola scorta dei lumi della propria individuale razionalità», ma ci si governa sulla base di costumi e istituzioni antiche, «patrimonio generale di esperienza accumulato

22. Ivi, p. 195 .

23. E. Burke, Ricorso dai nuovi agli antichi Whig, cit., p. 552.

24. E. Burke, Riflessioni sulla rivoluzione in Francia, cit., p. 194.

25. Ivi, p. 191. 
Metamorfosi dei

Lumi 10.

L'emergenza

del

sentimento

142 dai popoli nel corso di lunghi secoli». Non è un caso che Burke veda una continuità tra la Magna Charta e il Bill of Rights. C'è un ordine concreto, una costituzione materiale che non è frutto di pura scelta ma di «eredità». Proteggere la costituzione contro il cattivo governo ha significato infatti stare «alla presenza di antenati venerabili» ${ }^{26}$ e difendere un sistema in cui l'eccesso di una libertà astratta è temperato da «una gravità che ispira timore» ${ }^{27}$. Ecco perché sarebbe assurdo dire che nella costituzione inglese il monarca è legittimato dal popolo. Burke mostra come una delle preoccupazioni del Parlamento durante la Glorious Revolution fosse proprio quella di mantenere per quanto possibile intatta una linea ereditaria, fino a celare addirittura ogni parvenza di discontinuità con il passato - discontinuità che in Francia è ora rivendicata come un nuovo inizio. Il re d'Inghilterra è legittimo non perché autorizzato dal basso, ma proprio perché si situa in una linea che non esprime semplicemente l'eredità di una famiglia, quella reale, ma l'eredità del regno stesso, di cui la persona del monarca è solo l'architrave, la funzione di articolazione complessiva, e contro la quale nulla può la sua stessa volontà soggettiva. In questo contesto tutte le parti del corpo politico concorrono, per cui non si può dire che l'autorità stia astrattamente nel popolo o nel re, ma deve essere in equilibrato accordo tra corona, aristocrazia, camera dei comuni e popolo, erede quest'ultimo di diritti e privilegi determinati. Questo era secondo Burke anche lo spirito che presiedeva all'antica costituzione francese, composta proprio da una serie di elementi diversi e perfino opposti, capaci di produrre quella tensione positiva tra interessi e volontà che, di nuovo, «rendono la deliberazione una questione non di scelta ma di necessità» ${ }^{28}$.

Il sublime politico di Burke è quindi un sublime che non può e non deve essere assolutizzato, a dispetto di ogni interpretazione reazionaria e conservatrice del pensiero burkiano. Non si tratta di isolare nella sua purezza il sublime del despota e della sua radicale oscurità, perché l'autorità di chi governa è sempre presa nel movimento complessivo di principi differenti, incarnati non solo dalle diverse parti della 
società politica (nobiltà, clero, popolo); né semplicemente di insistere sulla commistione di fonti normative eterogenee radicate negli statuti e nel diritto comune. Si tratta prima ancora di dare rilevanza politica proprio a una tensione costituzionale che emerge in particolare nel rapporto sempre aperto tra monarchia e Parlamento: se il monarca incarna senza dubbio il momento del sublime, perché si rende garante dell'unità del regno, l'elemento della bellezza non è però spazzato via ma resta ineliminabile, perché tutta la particolarità concreta delle volontà politiche di cui i cittadini sono portatori - e che il contrattualismo riduce a semplice rappresentazione indifferenziata della totalità - trova un posto determinato nel sistema della rappresentanza per ordini di cui le camere parlamentari sono l'espressione, irriducibile ad alcuna unità immediata di una volontà sovrana.

Tutto il contrario accade nella Francia Rivoluzionaria. Essendo il potere dell'Assemblea incondizionato perché formalmente legittimo, non c'è più alcuna dimensione etica sostanziale nella quale possano agire i vincoli propri delle antiche costituzioni. Si è voluto fare della costituzione qualcosa di semplice, di poco complicato, di snello, piccolo - e dunque bello, se ricordiamo che la piccolezza e la chiarezza sono caratteri propri della bellezza - ma si è finito per istituire un potere terribile e privo di controllo che non ne rappresenta un effetto contingente ma una conseguenza inevitabile. Così nell'Assemblea Nazionale, «invece di trovarsi obbligati ad agire secondo una costituzione fissa, essi hanno il potere di fare una costituzione secondo i propri disegni. Non v'è niente in cielo o in terra che possa controllarli» ${ }^{29}$. Ne segue che «in una simile condizione di potere illimitato per propositi indefiniti e indefinibili, il danno proveniente da una inettitudine morale e quasi fisica dell'uomo a ricoprire tale funzione deve essere il più grande che si possa concepire nell'amministrazione delle cose umane» ${ }^{30}$. E quindi un potere che si pretende simile a quello di Dio, che si fa privo di regola e di confine, come quello del Leviatano - non a caso dotato dei caratteri di un deus mortalis - di cui Hobbes poteva dire che «non est potestas super terram quae comparetur

29. Ivi, p. 205.

30. Ivi, p. 206. 
Metamorfosi

dei

Lumi 10.

L'emergenza

del

sentimento ei». È il potere di una teologia politica, potremmo dire, che crede di rendere immanente alla presa della ragione umana la stessa potenza del divino e non mantiene rispetto a essa quello scarto e quell'eccedenza - quella reverenza - che sono invece propri del «diletto» costituzionale di cui parla Burke. Di nuovo, la pretesa di un bello assoluto ha prodotto un assoluto sublime.

\section{Conclusioni}

Che spazio occupa dunque l'emozione, per Burke, in un quadro propriamente politico? Sulla base delle considerazioni proposte, è senz'altro possibile dire che la riflessione costituzionale burkiana ci consente di riorientare in un senso specifico tutte quelle categorie osservate in precedenza: autopreservazione, amore, simpatia, imitazione, ambizione, sono altrettanti momenti dell'ordito etico della costituzione, che devono coesistere in una cornice di delight nella quale nessuno di essi possa diventare l'asse esclusivo dell'agire in comune. Il buon ordine costituzionale è anzitutto un ordine di simpatia in senso forte, cioè di concreta solidarietà tra parti differenziate di cui è sempre necessario pensare lo stare assieme e l'unificazione. Ma questo non è che l'architrave che consente di tenere assieme determinazioni plurali declinate anzitutto attraverso lo spazio dei legami familiari, la dimensione imitativa del pregiudizio e delle consuetudini, il continuo processo di distinzione sociale e di rappresentazione di istanze particolari dettato dalla passione dell'ambizione, infine le traiettorie delle libertà singolari che l'ordinamento consente continuamente di far emergere e preservare. Si tratta pertanto, dentro le coordinate del diletto politico che le strutture di governo della società devono produrre, di mantenere in equilibrio una molteplicità di tonalità emotive costitutive dell'agire in comune degli uomini senza che alcuna prenda il sopravvento sulle altre. Nella Francia della Rivoluzione, al contrario, proprio perché interamente centrata su una passione dominante - quella dell'autoconservazione degli individui e delle loro volontà - Burke vede tradursi l'autorità politica in un mero strumento di coercizione estrinseca che assume i tratti esclusivi dell'attonimento, della paura della morte e di una necessità completamente cieca. 
Metamorfosi

dei

Lumi 10.

L'emergenza

del

sentimento

\section{Indice dei nomi}

Addison, Joseph, 127

Alembert, Jean-Baptiste Le Rond d', $59,64 \mathrm{n}, 98,100$

Alfieri, Vittorio, XIII-XV

Ansel, Yves, 20n

Aristotele, 81, 93

Arnold, Gottfried, 124n

Assmann, Aleida, 114n

Assmann, Jan, 114n

Baasner, Frank, 21n

Bach, Carl Philipp Emanuel, 102, 104

Bach, Johann Sebastian, 99, 102

Baier, Annette C., 14, 17n

Baillie, Joanna, 88

Balestra, Dino, 33n

Balistreri, Maurizio, 3

Balzac, Honoré de, 20

Barelli, Ettore, 120n

Barrell, John, 75n

Barry, James, 77n

Bartolozzi, Francesco, 86

Batteux, Charles, 100

Baumgarten, Alexander Gottlieb, 84

Beaufils, Marcel, 105n

Bell, John, 86

Belwe, Andreas, 115n

Bernet, Anne, 76n
Bernier, François, 36

Berthier, Philippe, 20n

Beyle, Henry (Stendhal), Ix, 19-21, 23, 26-31, 33n, 60

Blake, William, 87n

Blanc, Jean-Bernard Le, 89

Bodemann, Eduard, 123n

Bodmer, Johann Jakob, 123

Boileau, Nicolas, 127

Bordeu, Theophile de, $52 \mathrm{n}$

Bourgeois, Frances, 86

Bourke, Richard, 134n

Boydell, John, 86

Brissenden, Robert Francis, 28n

Broadley, Alexander Meyrick, 87n

Brombert, Victor Henri, 20n

Burke, Edmund, XIn, XII, 84, 126-142, 144

Burroughs, Catherine, $88 \mathrm{n}$

Burwick, Frederick, 86n

Bussy-Rabutin, Roger de, 73

Caccini, Giulio, 95

Calprènede, Gautier de Coste de La, 71

Calzabigi, Simone Francesco Maria

Ranieri de', xIv, 102

Carlson, Julie A., 86n 


\section{Metamorfosi dei \\ Lumi 10. \\ L'emergenza \\ del \\ sentimento}

Carson, Craig, 137n

Cartesio (René Descartes), viI, xv, 29, $36,39,40,80,82,98$

Cassirer, Ernst, 109, 110

Cellier, Léon, 30n

Cerruti, Marco, xv

Chabanon, Michel-Paul-Guy de, x, 92, 93, 100-103

Charrier-Vozel, Marianne, $67 \mathrm{n}$

Chateaubriand, François-René, xıII, 20

Choiseul, duchessa di (LouiseHonorine Crozat du Châtel), 59

Cirillo, Domenico, XIII

Coe, Richard N., 20n

Coeffeteau, Nicolas, 36

Cohen, Margaret, 28n

Condillac, Étienne Bonnot de, 38n

Congreve, William, 88

Contarini, Silvia, $\mathrm{xv}$

Cooke, William, 82

Corbin, Alain, 35n

Corneille, Pierre, viII, 48

Corredor, Marie-Rose, 20n

Cortés, Jerónimo, 81

Corti, Claudia, 75, 83n, 84n, 86n, 87n

Coste, Pierre, 44n, 110n

Couperin, François, 97

Courtine, Jean-Jacques, 35n

Coventry, Angela M., 8n

Craven, Elizabeth, 87n

Craveri, Benedetta, 60-63, 65-69

Croce, Benedetto, 109

Crouzet, Michel, 19, 23

Cureau de La Chambre, Marin, 36

Deckard, Michael Funk, 133n

Deffand, Madame du (Marie de VichyChamrond), Ix, 59-74

Dehrmann, Mark-Georg, 111n, 124n

Del Litto, Victor, 19, 27n, 60n

Del Vecchio, Marcello, 139n

Delia, Luigi, $45 n$

Della Porta, Giovan Battista, 81

Denby, David J., 28n

Dennis, John, 127

Depont, Charles-Jean-François, 137

Destouches (Philippe Néricault), 27

Destutt de Tracy, Antoine-LouisClaude, 26

Di Benedetto, Arnaldo, XIv, xv

Diderot, Denis, VIII, XII, 25, 71, 100

Didier, Béatrice, 20n

Dieckmann, Herbert, 25n

Dilthey, Wilhelm, 109
Domenichino, Domenico Zampieri

(Dominiquin), 48

Du Bos, Jean-Baptiste, viI, VIII, 100n

Duisit, Lionel, 65, 68n, 70n, 71n

Duso, Giuseppe, 139n

Eagleton, Terry, 133n

Engel, Johann, 82, 86

Esquirol, Jean-Étienne Dominique, 50, $56 n$

Estève, Michel, 100

Fabre, Jean, 25n

Federico II di Hohenzollern, 122

Fontenelle, Bernard Le Bovier de, 96

Foote, Samuel, 82

Frey, Christiane, 124n

Furetière, Antoine, 36, 40n

Furniss, Tom, 137

Fuseli, Henry (Johann Heinrich Füssli), 75-77, 79, 86, 87

Gagliano, Marco da, 95

Gagnebin, Bernard, 21n, 66n, 94n

Gagnon, Henri, 26

Gall, Franz Joseph, 49

Garrick, David, 83

Gassendi, Pierre, 36, 39

Gauchet, Marcel, 54n

Gaukroger, Stephen, 21n

Gerard, Alexander, 85

Giacomo I (Giacomo Stuart), 140

Gill, Miranda, 30n

Gillray, James, 89

Giorgio III (Giorgio Guglielmo Federico di Hannover), 122

Gluck, Christoph Willibald, XI, 102

Goring, Paul, 21n

Greco, Lorenzo, 5n, 13n

Groult, Martine, 45n

Grozio, Ugo (Huig de Groot), 113

Guglielmi, Giuseppe, 32n

Guilleragues, Gabriel de, 72n

Hall, John, 127

Haller, Albrecht von, 122

Hamann, Johann Georg, 112

Hastings, Warren, 138

Haug, Walter, 114n

Hazlitt, William, 75n, 77n

Hegel, Georg Wilhelm Friedrich, 139

Helvétius, Claude-Hadrien, 27

Hénault, Charles-Jean-François, $x$, 60-65

Hill, Aaron, 81 
Hobbes, Thomas, 4, 5, 8, 12, 39n, 137, 139, 143

Hogarth, William, 82, 84, 85, 89

Holcroft, Thomas, 84

Home, Henry, 85

Huet, Jean-Yves, 73n

Hugo, Victor, IX, 20

Hume, David, 80, 81n, 133

Hummel, Carz, 124n

Hunt, John, 90

Hunt, Leigh, 78

Hunt, Lynn, 71n

Hutcheson, Francis, 4, 6, 7n, 12, 84, 112,113

Im Hof, Ulrich, 113n

Jaucourt, Louis de, 21, 37, 41n, 43n

Kail, Peter J., 7n

Kant, Immanuel, 114-116, 132

Keats, John, 85

Kemble, John Philip, 83

Kenny, Shirley Strum, 75n

Klein, Lawrence E., 4n

Klerks, William, 69n

Knowles, John, 79n, 87n

La Bruyère, Jean de la, 43, 44n

La Cépède, conte di (Bernard Germain

Étienne de Laville), x, 92, 100n

Lacretelle, Pierre de, 37n, 38, 40-44

Lacretelle, Jean de, $37 \mathrm{n}$

Laffont, Robert, 105n

Lancelin, Pierre-François, 27n

Larson, Kenneth E., 70n

Lavater, Johann Kaspar, 85, 86

Le Brun, Charles, 46n, 80

Le Grand, Ernest G., 67n

Lecaldano, Eugenio, 3, 6n, 8n, 12n

Lecerf de la Viéville, Jean-Laurent, 96

Leibniz, Gottfried Wilhelm von, vII, $\mathrm{XI}, 110$

Leonzio, Elisa, 109

Leopardi, Giacomo, xIII

Lespinasse, Julie, 59, 63

Locke, John, 12

Longino, Quinto Cassio, 127

Macfie, Alexander Lyon, 4n

Macklin, Thomas, 86

Malebranche, Nicolas, vII

Mall, Laurence, 23n

Mandeville, Bernard de, 18, 113

Maria Antonietta (Maria Antonia
Giuseppa Giovanna d'AsburgoLorena), 138

Martelloni, Anna, 138n

Martineau, Henri, 30n

Maupertuis, Pierre-Louis Moreau de, 59

Maurois, André, 68n

Mautner, Thomas, 112n

May, Gita, 20n

Melville, Lewis, 87n

Mendelssohn, Jakob Ludwig Felix, 112

Menin, Marco, xv, 19, 23n, 25n, 29n

Mercier, Louis-Sébastien, 27n

Merot, Alain, 46n

Messina, Maria Grazia, 75n

Messina, Simone, xv

Michelangelo (Michelangelo Buonarroti), 79

Miglietta, Goffredo, 127n

Milton, John, 79

Mitchell, Thomas, 134, 137

Montesquieu (Charles-Louis de Secondat), 59, 64n

Monteverdi, Claudio, 95

Monti, Vincenzo, xv

Moreau de la Sarthe, Jacques Louis, 45, $47-50,52-56$

Mozart, Wolfgang Amadeus, XI, 102, 104

Neocleous, Mark, 137n

Nerlich, Michael, 20n

Newton, Isaac, 3

Nidditch, Paul H., 6n, 81n

Nietzsche, Friedrich, 19

Northcote, James, 78

Nouvel, Walter, 91n

O'Neill, Daniel, 133n

Obereit, Jacob Hermann, 124n

Opie, Johnx, 76-77

Paine, Thomas, 138

Parini, Giuseppe, XIII

Parsons, James, 81n, 82

Pascal, Gabrielle, 30n

Pascoe, Judith, 83n

Peri, Jacopo, 95

Persio (Aulo Persio Flacco), 120

Piirimäe, Eva, 112n

Pindemonte, Giovanni, xv

Pindemonte, Ippolito, XIII

Pinel, Philippe, 55,56

Piva, Franco, 21n

Platone (Platon), 93, 94, 97, 111 
Metamorfosi dei

Lumi 10.

L'emergenza del sentimento
Pointon, Marcia, 83n

Pope, Alexander, XIII

Poussin, Nicolas, 47, 48

Prévost, Pierre, 27n

Price, Richard, 126, 137

Proust, Jacques, 27n

Pulteney, John, 127

Raffaello, Raphaël (Raffaello Sanzio), 48, 49, 79

Raphael, David Daiches, 4n

Rageot, Gaston, 70n

Rameau, Jean-Philippe, x, 99, 100

Raymond, Marcel, 21n, 66n, 94n

Reynolds, Joshua, 47, 75n

Richardson, Samuel, 25, 28n, 66, 71

Riskin, Jessica, 21n

Ritchie, Daniel E., 137n

Ritchie, Fiona, 70n

Roach, Joseph R., 83n

Rousseau, Jean-Jacques, VII, Ix, XIII, 19-30, 33n, 44, 50, 60, 66, 73, 94, 99-101, 113, 133, 137

Rowlandson, Thomas, 89

Rustighi, Lorenzo, 126

Sabor, Peter, 70n

Saby, Pierre, 91

Sager, Angela M., 8n

Sainte-Beuve, Charles Augustin de, 59

Sakakura, Yûji, 23n

Salfi, Francesco Saverio, XV

Santerre, Jean-Baptiste, 49

Sauder, Gerhard, 21n

Savonarola, Michele, 81

Scheffer, Ulrik, 59

Schelle, Hansjörg, 70n

Schings, Hans-Jürgen, 125n

Schleiermacher, Friedrich Daniel Ernst, 109

Schmidt, Alexander, 112n, 113n

Scudéry, Madeleine de (Mademoiselle de Scudéry), 71-73

Sedaine, Michel-Jean, 71

Selby-Bigge, Lewis Amherst, 80n, 81n

Senault, Jean-François, 36

Sévigné, Madame de (Marie de Rabutin-Chantal), 73,74

Shaftesbury (Anthony Ashley-Cooper), $\mathrm{XI}, 4 \mathrm{n}, 6,12,84,85,109-113,117$ 125,127
Shakespeare, William, 70, 79, 86n

Sicco, Debora, 59

Siddons, Henry, 86

Siddons, Sarah Kemble (Mrs. Siddons), 86

Signoret, Véronique, 45n

Smith, Adam, 4n, 9n, 12, 13, 42

Spandri, Francesco, 20n

Spinoza, Baruch, viI, 40n, 109

Starobinski, Jean, 32n, 101n

Staum, Martin S., 55n

Stendhal (Henry Beyle), Ix, 19-21, 23, 26-31, 33n, 60

Stravinskij, Igor' Fëdorovič (Stravinsky), 91, 105

Swain, Gladys, 54n

Tacito, Publio Cornelio (Tacite), 26

Talon-Hugon, Carole, 35n, 36n, 39n

Taraborrelli, Angela, 4n

Taylor, Jacqueline, 5n, 12n

Teysseire, Daniel, 35

Tommaso d'Aquino (Thomas d'Aquin), $35 n$

Trivero, Paola, 8, 15

Trousson, Raymond, 19n, 20n, 28

Turco, Luigi, 4n

Vaccari, Alessio, 7n, 8n, 14

Varloot, Jean, 25n

Vauvenargues, Luc de Clapiers de, 43

Vermeir, Koen, 133n

Verri, Alessandro, xIV

Vigarello, Georges, 35n

Vitz, Rico, 7n

Vivaldi, Antonio, 93, 95, 96

Voltaire (François-Marie Arouet), 59, $61,64-66,70-72$

Walpole, Horacex, 59-74

Weltman-Aron, Brigitte, 23n

West, Shearer, 83n

Wieland, Christoph Martin, 124n

Wilde, Samuel de, 86

Wittler, Kathrin, 113n

Wornum, Ralph Nicholson, 77n

Worthen, William, 83n

Zimmermann, Johann Georg, xI, 109, $112,122-125$ 
Metamorfosi

dei

Lumi 10.

L'emergenza del

sentimento

\section{Gli autori}

aA

Maurizio Balistreri, Università degli Studi di Torino

Claudia Corti, Università degli Studi di Firenze

Elisa Leonzio, Freie Universität Berlin

Marco Menin, Università degli Studi di Torino

Lorenzo Rustighi, Università degli Studi di Padova

Pierre Saby, Université Lumière - Lyon 2

Debora Sicco, Università degli Studi del Piemonte Orientale

Daniel Teysseire, Université de Caen Normandie

Paola Trivero, Università degli Studi di Torino 


finito di stampare

da Digitalandcopy, Milano per i tipi di

Accademia University Press

in Torino

nel mese di dicembre 2020 